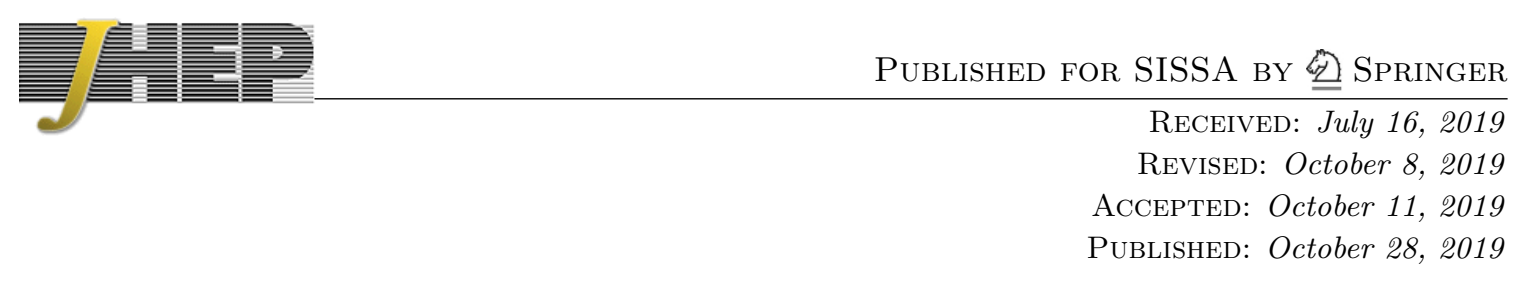

\title{
Mass spectrum of the hidden-charm pentaquarks in the compact diquark model
}

\author{
Ahmed Ali, ${ }^{a, 1}$ Ishtiaq Ahmed, ${ }^{b}$ M. Jamil Aslam, ${ }^{c}$ Alexander Ya. Parkhomenko ${ }^{d}$ and \\ Abdur Rehman ${ }^{b}$ \\ ${ }^{a}$ Deutsches Elektronen-Synchrotron DESY, \\ D-22607 Hamburg, Germany \\ ${ }^{b}$ National Centre for Physics, Quaid-i-Azam University Campus, \\ Islamabad 45320, Pakistan \\ ${ }^{c}$ Physics Department, Quaid-i-Azam University, \\ Islamabad 45320, Pakistan \\ ${ }^{d}$ P. G. Demidov Yaroslavl State University, \\ Sovietskaya 14, 150003 Yaroslavl, Russia \\ E-mail: ahmed.ali@desy.de, ishtiaqmusab@gmail.com, \\ muhammadjamil.aslam@gmail.com, parkh@uniyar.ac.ru, \\ Abdur.Rehman@fuw . edu.pl
}

ABSTRACT: The LHCb collaboration have recently updated their analysis of the resonant $J / \psi p$ mass spectrum in the decay $\Lambda_{b}^{0} \rightarrow J / \psi p K^{-}$, making use of their combined Run 1 and Run 2 data. In the updated analysis, three narrow states, $P_{c}(4312)^{+}, P_{c}(4440)^{+}$, and $P_{c}(4457)^{+}$, are observed. The spin-parity assignments of these states are not yet known. We interpret these narrow resonances as compact hidden-charm diquark-diquarkantiquark pentaquarks. Using an effective Hamiltonian, based on constituent quarks and diquarks, we calculate the pentaquark mass spectrum for the complete $\mathrm{SU}(3)_{F}$ lowest $S$ and $P$-wave multiplets, taking into account dominant spin-spin, spin-orbit, orbital and tensor interactions. The resulting spectrum is very rich and we work out the quark flavor compositions, masses, and $J^{P}$ quantum numbers of the pentaquarks. However, heavy quark symmetry restricts the observable states in $\Lambda_{b}$-baryon, as well as in the decays of the other weakly-decaying $b$-baryons, $\Xi_{b}$ and $\Omega_{b}$. In addition, some of the pentaquark states are estimated to lie below the $J / \psi p$ threshold in $\Lambda_{b}$-decays (and corresponding thresholds

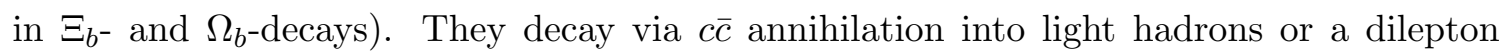
pair, and are expected to be narrower than the $P_{c}$-states observed. We anticipate their discovery, as well as of the other pentaquark states present in the spectrum at the LHC, and in the long-term future at a Tera- $Z$ factory.

KeYwords: Phenomenological Models, QCD Phenomenology

ARXIV EPRINT: 1907.06507

${ }^{1}$ Corresponding author. 


\section{Contents}

1 Introduction 1

2 Doubly-heavy triquark - light diquark model of pentaquark 6

3 Effective Hamiltonian for pentaquark spectrum 9

4 Mass formulae for pentaquark spectrum 12

$\begin{array}{lll}4.1 & S \text {-wave pentaquarks } & 12\end{array}$

$4.2 P$-wave pentaquarks 16

5 Hidden-charm pentaquark mass predictions 20

5.1 Input parameters 20

5.2 Predictions for the hidden-charm unflavored pentaquarks 23

$\begin{array}{lll}5.3 & \text { Comparisons with the dynamical diquark model } & 27\end{array}$

5.4 Mass predictions for the strange hidden-charm pentaquarks 28

6 Pentaquark decay widths 33

$\begin{array}{lll}7 & \text { Conclusions } & 39\end{array}$

A Corrections due to spin-spin interactions between the constituents of doubly-heavy triquark and light diquark 41

B Mass derivations for the $\boldsymbol{P}$-wave pentaquark states 46

B.1 The states with $J^{P}=3 / 2^{+}$and triquark spin $S_{t}=3 / 2 \quad 46$

B.2 The states with $J^{P}=3 / 2^{+}$and triquark spin $S_{t}=1 / 2$

B.3 The states with $J^{P}=1 / 2^{+}$and triquark spin $S_{t}=1 / 2 \quad 49$

C $\chi^{2}$-analysis of orbitally-excited $\Omega_{c}^{*}$-baryons $\quad 50$

\section{Introduction}

Recently, the LHCb collaboration has presented an updated account of the resonant $J / \psi p$ mass spectrum in the decay $\Lambda_{b}^{0} \rightarrow J / \psi p K^{-}$, based on the combined Run 1 and Run 2 data, adding up to $9 \mathrm{fb}^{-1}$ [1]. In this analysis, which supersedes their earlier findings from 2015 [2], nominal fits of the data have been performed with an incoherent sum of BreitWigner amplitudes, which have resulted in the observation of three peaks, whose masses, decay widths (with $95 \%$ C.L. upper limits), and the ratio $\mathcal{R}$, defined as

$$
\mathcal{R} \equiv \frac{\mathcal{B}\left(\Lambda_{b} \rightarrow P_{c}^{+} K^{-}\right) \mathcal{B}\left(P_{c}^{+} \rightarrow J / \psi p\right)}{\mathcal{B}\left(\Lambda_{b} \rightarrow J / \psi p K^{-}\right)},
$$


are given in table 1 . The state $P_{c}(4450)^{+}$in the 2015 data [2], is now replaced by two narrow states, $P_{c}(4440)^{+}$and $P_{c}(4457)^{+}$. In addition, a third narrow peak, $P_{c}(4312)^{+}$, having the mass $M=\left(4311.9 \pm 0.7_{-0.6}^{+6.8}\right) \mathrm{MeV}$, is also observed. The spin-parity, $J^{P}$, assignments of the three narrow states, which are crucial to decipher the underlying dynamics of the pentaquark states, are not yet determined. The broad peak $P_{c}(4380)^{+}$from the earlier data [2] is neither confirmed nor refuted, as the current LHCb analysis is not sensitive to broad resonances. Hence, it is entirely conceivable that more $P_{c}^{+}$-like structures are present in the $J / \psi p$ invariant-mass spectrum, anticipated in the compact pentaquark interpretation, similar to the excited $\Lambda^{*}$-baryon spectrum in the $K p$ channel, expected in the quark model and confirmed in data [3]. For the discussion of the $2015 \mathrm{LHCb}$ data on pentaquarks and other multiquark hadrons and references to the earlier works see the reviews [3-14] and [15].

The new pentaquarks reported by LHCb [1] have triggered a surge in theoretical papers interpreting the three narrow resonances as loosely-bound hadronic-molecule states in various incarnations [16-25]. A more up to date list of references can be seen in the proceedings of a recent Workshop on Exotic Hadrons [26]. Indeed, in the mass region of interest, a number of kinematical thresholds are present, such as $\Sigma_{c}^{+} \bar{D}^{0}\left(E_{\mathrm{thr}}=4317.73 \pm 0.41 \mathrm{MeV}\right)$, $\Sigma_{c}^{*+} \bar{D}^{0}\left(E_{\mathrm{thr}}=4382.3 \pm 2.4 \mathrm{MeV}\right), \Lambda_{c}^{*+} \bar{D}^{0}\left(E_{\mathrm{thr}}=4457.09 \pm 0.35 \mathrm{MeV}\right)$, and $\Sigma_{c}^{+} \bar{D}^{* 0}$ $\left(E_{\mathrm{thr}}=4459.9 \pm 0.9 \mathrm{MeV}\right)[3]$. The masses of the observed resonances in the hadron molecule interpretation are essentially the sums of their respective hadronic constituents, which is striking. Thus, not only the three observed narrow pentaquarks can be accommodated, having the spin-parity and isospin $\left(J^{P}=1 / 2^{-}, I=1 / 2\right)$ and $\left(J^{P}=3 / 2^{-}, I=1 / 2\right)$, but several other states, also with a negative parity due to their assumed $S$-wave character, are predicted. These emphatic claims are at best tentative and await a confirmation of the spin-parity assignments of the three observed states. The nature of the $P_{c}(4312)^{+}$as a candidate for the $\Sigma_{c}^{+} \bar{D}$ molecular state was also analyzed based on the $S$-matrix principles [27] and it was found that the attraction in this system is not enough for a bound state.

We emphasize that the decays $\Lambda_{b} \rightarrow \Sigma_{c}^{(*)}+X$ are anticipated to be suppressed by Heavy Quark Symmetry (HQS), due to the mismatch of the spectator diquark spin (a conserved quantity in the HQS limit) in $\Lambda_{b^{-}}$and $\Sigma_{c}^{(*)}$-baryons. This suppression is wellknown, but is often ignored in the literature on the hadron molecular approach for the new $P_{c}$-states, though there are some rare exceptions, see, for example, [28]. There is an ample evidence of this suppression in the PDG tables, in which the non-leptonic transitions $\Lambda_{b} \rightarrow \Lambda_{c}^{(*)}+X$ dominate and the $\Lambda_{b} \rightarrow \Sigma_{c}^{(*)}+X$ transitions are rather sparse [3]. In the exact HQS limit, such decays are forbidden, but as the HQS is not an exact symmetry and is broken by power $\left(1 / m_{b}\right)$ and QCD $\left(\mathcal{O}\left(\alpha_{s}\left(m_{b}\right)\right)\right)$ corrections, they are allowed but have reduced decay rates. It has important implications for the analysis of the $P_{c}$-states in the hadron molecule approach as well. In particular, it implies that the branching ratios $\mathcal{B}\left(\Lambda_{b} \rightarrow P_{c}^{+} K^{-}\right)$, which in the hadron-molecule interpretations of the $P_{c^{-}}$-states are supposed to be induced by the intermediate $\left(\Sigma_{c} \bar{D}^{(*)}\right) K^{-}$states, followed by rescattering, are subject to the HQS-implied suppression. Since the ratio $\mathcal{R}$ in (1.1), which is the product $\mathcal{B}\left(\Lambda_{b} \rightarrow P_{c}^{+} K^{-}\right) \times \mathcal{B}\left(P_{c}^{+} \rightarrow J / \psi p\right)$ in units of $\mathcal{B}\left(\Lambda_{b} \rightarrow J / \psi p K^{-}\right)$, is well-measured for all three states by $\mathrm{LHCb}$ (see table 1 ), the branching ratios $\mathcal{B}\left(P_{c}^{+} \rightarrow J / \psi p\right)$ are not 


\begin{tabular}{|ccccc|}
\hline State & Mass $[\mathrm{MeV}]$ & Width $[\mathrm{MeV}]$ & $(95 \% \mathrm{CL})$ & $\mathcal{R}[\%]$ \\
\hline$P_{c}(4312)^{+}$ & $4311.9 \pm 0.7_{-0.6}^{+6.8}$ & $9.8 \pm 2.7_{-4.5}^{+3.7}$ & $(<27)$ & $0.30 \pm 0.07_{-0.09}^{+0.34}$ \\
$P_{c}(4440)^{+}$ & $4440.3 \pm 1.3_{-4.7}^{+4.1}$ & $20.6 \pm 4.9_{-10.1}^{+8.7}$ & $(<49)$ & $1.11 \pm 0.33_{-0.10}^{+0.22}$ \\
$P_{c}(4457)^{+}$ & $4457.3 \pm 0.6_{-1.7}^{+4.1}$ & $6.4 \pm 2.0_{-1.9}^{+5.7}$ & $(<20)$ & $0.53 \pm 0.16_{-0.13}^{+0.15}$ \\
\hline
\end{tabular}

Table 1. Masses, decay widths (with $95 \%$ C.L. upper limits), and the ratio $\mathcal{R}$, of the three narrow $J / \psi p$ resonances observed by the LHCb collaboration in the $\Lambda_{b} \rightarrow J / \psi p K^{-}$decay [1].

expected to be small in the hadron-molecule interpretation. It is relevant to point out that model-dependent $90 \%$ C.L. upper limits on $\mathcal{B}\left(P_{c}^{+} \rightarrow J / \psi p\right)$ of $4.6 \%, 2.3 \%$, and $3.8 \%$ for $P_{c}^{+}(4312), P_{c}^{+}(4440)$, and $P_{c}^{+}(4457)$, respectively, have been posted by the GlueX collaboration [29], assuming they have $J^{P}=3 / 2^{-}$(i.e., $S$-wave $J / \psi p$ states). While this is currently not robust enough an argument against the $P_{c}$ 's being hadron molecules, due to inherent assumptions about the theoretical estimates of the photoproduction cross sections, but improved measurements will soon test this interpretation in the $P_{c}$-photoproduction experiments $[29,30]$. The role of the photoproduction process $\gamma p \rightarrow J / \psi p$ in constraining the models of the new $P_{c}$-states is noted in [31, 32]. Another process to measure the $P_{c}$ production is the antiproton-deuterium collisions, in which case the charm quark and antiquark entering the charmonium state $J / \psi$ or $\eta_{c}$ are the result of the proton-antiproton annihilation [33]. Thus, the internal consistency of the hadron molecule approach for the new $P_{c}$ states remains to be checked.

In the preceding Letter [34], two of us have argued that there also exists a prima facie case for the three narrow $P_{c}^{+}$-resonances to be considered as candidates for compact pentaquarks. The basic idea of this approach is that highly correlated colored diquarks play a key role in the physics of multiquark states [35-37], and they are at work in the underlying dynamics of the $P_{c}^{+}$-states. A brief account of the new pentaquarks was presented in [34] in the effective Hamiltonian framework based on constituent quarks and diquarks, using isospin and heavy quark symmetry. In this paper, we extend the compact diquark template to cover the complete $\mathrm{SU}(3)_{F}$-multiplets of compact hidden-charm pentaquarks in the lowest $S$ - and $P$-wave states. Some of them can be searched for in the decays of the $\Lambda_{b^{-}}$ baryon, but some others can only be reached in the decays of the other weakly-decaying b-baryons, $\Xi_{b}$ and $\Omega_{b}$.

The pentaquark dynamics depends upon how the five constituents, i.e., the 4 quarks and an antiquark, are structured. Since quarks transform as a triplet 3 of the color SU(3)group, the diquarks resulting from the direct product $3 \otimes 3=\overline{3} \oplus 6$, are thus either a color anti-triplet $\overline{3}$ or a color sextet 6 . Of these only the color $\overline{3}$ configuration is kept, as suggested by perturbative arguments. Another justification is that the color sextet diquarks are heavier than the corresponding color anti-triplet ones, and can be integrated out for spectroscopic considerations. This remains to be quantified, which can be eventually done in lattice QCD. Both spin-0 and spin-1 diquarks are, however, allowed. In the case of a diquark $\left[q^{\prime} q^{\prime \prime}\right]$ consisting of two light quarks $\left(q^{\prime}, q^{\prime \prime}=u, d, s\right)$, the spin-0 diquarks are believed to be more tightly bound than the spin- 1 , and this hyperfine splitting has 
implications for the spectroscopy. For the heavy-light diquarks, such as $[c q]$ or $[b q]$, this splitting is suppressed by $1 / m_{c}$ for the $[c q]$-diquark or by $1 / m_{b}$ for the [bq]-diquark, and hence both spin configurations are treated at par. Thus, the constituents of the hiddencharm pentaquarks in the compact diquark model are $[c q]_{\overline{3}}, \bar{c}_{\overline{3}}$, and $\left[q^{\prime} q^{\prime \prime}\right]_{\overline{3}}$, which make up a color singlet. However, it is still a three-body problem to solve and there are several dynamical possibilities to model their interconnections. We follow here the intuitive picture in which the heavier components form a nucleus and the lighter one is in an orbit around this nucleus, as it is energetically easier to excite light degrees of freedom. Among the three constituents, the light diquark $\left[q^{\prime} q^{\prime \prime}\right]_{3}$ is the lightest. Moreover, its spin-parity quantum numbers are fixed due to heavy-quark symmetry constraints. So, we keep the light diquark as emerging intact in the $b$-baryon decays, and put it in the orbit for the $P$-wave states, with the heavier components, carrying a charm quark or a charm antiquark, acting as a nucleus of (an almost) static color source. This is shown in figure 1 and the color and flavor structure in this approach can be written as $\left[\left[\bar{c}_{\overline{3}}(c q)_{\overline{3}}\right]_{3}\left(q^{\prime} q^{\prime \prime}\right)_{\overline{3}}\right]$. Such a description is closer to the doubly-charm tetraquarks, with the quark content $\left[(\bar{c} \bar{c})_{3}\left(q^{\prime} q^{\prime \prime}\right)_{\overline{3}}\right]$, and doublycharm antibaryons, having $\left[(\bar{c} \bar{c})_{3} \bar{q}_{\overline{3}}\right]$, as all three systems have two charm (anti)quarks at the center [38-49]. We note that a colored diquark-triquark template for pentaquarks $\bar{\theta} \delta \equiv\left[\bar{Q}\left(q_{1} q_{2}\right)_{\overline{3}}\right]_{3}\left(Q q_{3}\right)_{\overline{3}}$, through the color-triplet binding mechanism, was first used by Lebed [50]. There is also the third possibility to introduce a model for the internal structure of a pentaquark $[51,52]$ in which the light and heavy diquarks bind first into a tetraquark in a color-triplet 3 state which further interacts with the charm antiquark, $\left[\left[(c q)_{\overline{3}}\left(q^{\prime} q^{\prime \prime}\right)_{\overline{3}}\right]_{3} \bar{c}_{\overline{3}}\right]$. Which of the three internal structures discussed provides a realistic template for the bound charm-anticharm pentaquarks remains to be seen.

In this paper we use the $L-S$ scheme for describing the angular structure of pentaquarks. The spin of the heavy $[c q]$-diquark is $\mathbf{S}_{h d}=\mathbf{S}_{c}+\mathbf{S}_{q}$ with the allowed values $S_{h d}=0,1$, where $\mathbf{S}_{c}$ and $\mathbf{S}_{q}$ are the spins of the charm- and light $u$-, $d$ - or $s$-quark, respectively. Combining the heavy diquark spin with the spin $\mathbf{S}_{\bar{c}}$ of the charm antiquark, we get the spin of the doubly-heavy triquark, $\mathbf{S}_{t}=\mathbf{S}_{h d}+\mathbf{S}_{\bar{c}}$, having the values $S_{t}=1 / 2,3 / 2$. The spin of the light $\left[q^{\prime} q^{\prime \prime}\right]$-diquark is $\mathbf{S}_{l d}=\mathbf{S}_{q^{\prime}}+\mathbf{S}_{q^{\prime \prime}}$, where $S_{l d}=0$, 1 . The total spin of the pentaquark is the sum of the triquark and the light-diquark spins, $\mathbf{S}=\mathbf{S}_{t}+\mathbf{S}_{l d}$ with the set of values $S_{t}=1 / 2,3 / 2,5 / 2$. In the case of the orbitally excited pentaquarks, the orbital angular momentum $\mathbf{L}$ of the pentaquark is the sum of two terms: $\mathbf{L}_{t}$, arising from the triquark system, and $\mathbf{L}_{\mathbf{l d}}$, which determines the relative motion of the light diquark around the doubly heavy triquark. The total orbital angular momentum $\mathbf{L}$ of the pentaquark is then obtained with the help of the momentum sum rules from quantum mechanics, i.e., it takes a value in the range $L=\left|L_{t}-L_{l d}\right|, \ldots, L_{t}+L_{l d}$. After $\mathbf{L}$ and $\mathbf{S}$ are constructed, the total angular momentum $\mathbf{J}$ of the pentaquark follows from $\mathbf{J}=\mathbf{L}+\mathbf{S}$. The parity of the states depends on the orbital angular momentum as $P=(-1)^{L+1}$. It is negative for the $S$-wave states and positive for the states with one unit of the orbital angular momentum.

Mass estimates are worked out in an effective Hamiltonian approach, which apart from the constituent quark and diquark masses, includes dominant spin-spin, spin-orbit, orbital, and tensor interactions. The effective Hamiltonian for the $S$-wave states in this formulation is the same as the one used earlier in the analysis of the $2015 \mathrm{LHCb}$ data 
on pentaquarks $[51,52]$, but the $P$-wave (and higher orbital angular) states are packaged differently. In addition to this, we incorporate the tensor interaction in the effective Hamiltonian, which affect the $P$-states, and work out the detailed mass spectrum in this framework, using the parameters fixed from earlier studies of baryons and hidden-charm tetraquarks. The resulting pentaquark spectrum is very rich. However, imposing the spin conservation in the heavy-quark symmetry limit, we argue that only that part of the pentaquark spectrum is expected to be observed in $\Lambda_{b}$-decays, in which the pentaquarks have a "good" light diquark, i.e., having spin $S_{l d}=0$. This reduces the number of anticipated pentaquark states in $\Lambda_{b}$-decays greatly. The same is also true for the decays of the $\Xi_{b}^{0,-}$-baryons which are from the same $\mathrm{SU}(3)_{F}$-triplet as the $\Lambda_{b}$-baryon. The other weakly decaying $b$-baryon, $\Omega_{b}$, in which the light diquark has the spin-1, belongs to the $\mathrm{SU}(3)_{F}$-sextet. Its decays will yield pentaquarks from the $\mathrm{SU}(3)_{F}$-decuplets, i.e., have a spin-1 light diquark $\left(S_{l d}=1\right)$ as a constituent $[51,53]$. Some of the unflavored pentaquark states are estimated to lie below the $J / \psi p$ (even below $\eta_{c} p$ ) threshold and they will decay via the $c \bar{c}$-annihilation into light hadrons or a lepton pair $\left(e^{+} e^{-}\right.$and $\left.\mu^{+} \mu^{-}\right)$, giving rise to states narrower than the observed peaks, which can also be searched in the $P_{c}^{+} \rightarrow p \ell^{+} \ell^{-}$ modes in $\Lambda_{b} \rightarrow P_{c} K^{-}$decays. The same holds for the decays of some other pentaquark

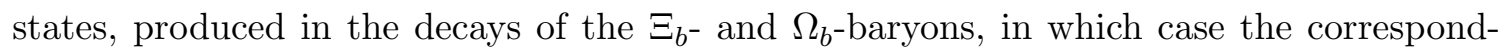
ing pentaquarks with one, two, or three strange quarks will give rise to narrow $\Lambda^{0} \ell^{+} \ell^{-}$, $\Xi^{\prime 0} \ell^{+} \ell^{-}$, and $\Omega^{-} \ell^{+} \ell^{-}$final states, respectively.

We also note that a detailed spectrum of the tetraquark and pentaquark states has also been worked out in the dynamical diquark model [54], using Born-Oppenheimer potentials calculated numerically on the lattice. Likewise, the $P_{c}^{+}$-states have been studied in different color-bindings, such as $\{(c \bar{c})((u d) u) ; L=0\}$ [28] and as $(c \bar{c})(u u d)$ hadrocharmonia [55].

This paper is organized as follows. In section 2 , we introduce the doubly-heavy triquark - light diquark model of pentaquark, and define the state vectors having a total angular momentum quantum number $J$ by $\left|S_{h d}, S_{t}, L_{t} ; S_{l d}, L_{l d} ; S, L\right\rangle_{J}$, where $S_{h d}, S_{t}$, and $S_{l d}$ are the spins of the heavy diquark, doubly-heavy triquark and light diquark, respectively. The corresponding sets of the $S$-wave state vectors (with $L_{t}=L_{l d}=L=0$ ) with the "good" $\left(S_{l d}=0\right)$ and "bad" $\left(S_{l d}=1\right)$ light diquarks are presented in tables 2 and 3, respectively. State vectors of the $P$-wave pentaquarks with the ground-state triquark $\left(L_{t}=0, L_{l d}=\right.$ $L=1$ ) and "good" light diquark with the spin $S_{l d}=0$ ("bad" light diquark with the spin $S_{l d}=1$ ), are given in table 4 (table 5). In section 3 , we give the effective Hamiltonian used to work out the pentaquark mass spectrum. In section 4, analytical expressions for effective Hamiltonian matrix elements are presented taking into account the dominant spin-spin interactions in the heavy and light diquarks and in the hidden-charm triquark. For the $P$-wave states, additional contributions and mixings due to the orbital, spin-orbit and tensor interactions are included. In all the cases, we diagonalize the mass matrices and the analytical equations for all the $S$ - and $P$-wave pentaquark masses are presented. Section 5 contains the values of various input parameters, with the constituent diquark masses given in table 6 and the spin-spin couplings, $\mathcal{K}_{\bar{Q} Q^{\prime}}$ and $\left(\mathcal{K}_{Q Q^{\prime}}\right)_{\overline{3}}$, extracted from spectra of mesons and baryons, are given in table 7. Our predictions for the unflavored pentaquark masses having the quark flavors $\left(\bar{c}[c q]\left[q^{\prime} q^{\prime \prime}\right]\right)$, with $q, q^{\prime}$, and $q^{\prime \prime}$ being $u$ - and 


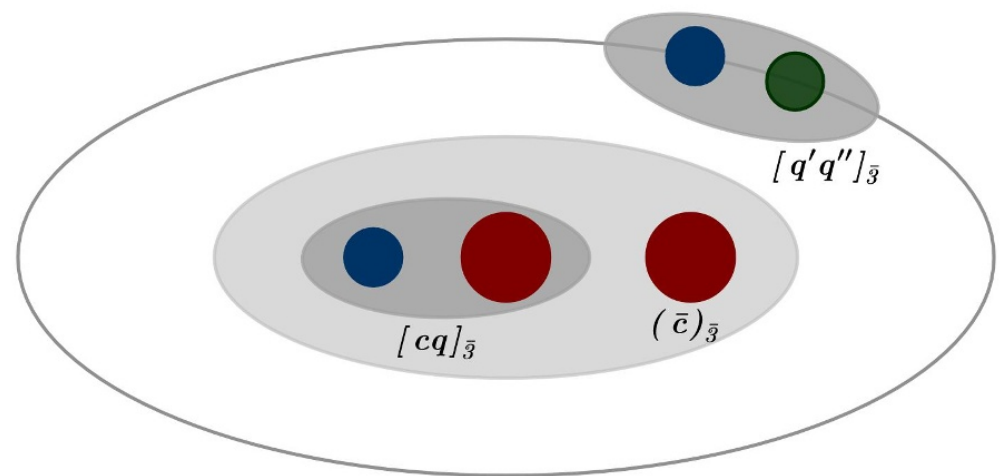

Figure 1. A picture of pentaquarks in the diquark model involving the heavy diquark $[c q]_{\overline{3}}$ and charm antiquark $(\bar{c})_{\overline{3}}$, which form the triquark system, which combines with the light diquark $\left[q^{\prime} q^{\prime \prime}\right]_{\overline{3}}$ to make a color singlet pentaquark. The subscripts indicate that all three constituents are color anti-triplets; $q, q^{\prime}$, and $q^{\prime \prime}$ are light quarks each of which can be $u-, d-$, or $s$-quark.

$d$-quarks, assuming the isospin symmetry, are given in table 8. Masses of the hiddencharm strange pentaquarks are shown in tables 10-14. In this section, a comparison of our approach with the dynamical diquark model [54] is also presented. We continue with a discussion of dominant decay channels of ground-state pentaquarks containing a scalar light diquark in section 6 and conclude in section 7. In appendix A, corrections due to the spin-spin interactions between the constituents of the doubly-heavy triquark and the light diquark are detailed; in appendix $\mathrm{B}$, cumbersome mass derivations for the $P$-wave pentaquark states are presented, and in appendix $\mathrm{C}$ the details and results of the $\chi^{2}$-analysis of the orbitally-excited $\Omega_{c}^{*}$-baryons are shown.

\section{Doubly-heavy triquark — light diquark model of pentaquark}

In the pentaquark picture shown in figure 1, there are two color flux tubes, with the first stretched between the charm diquark and the charm antiquark from which the diquark is in the color-antitriplet state $\overline{3}$. With the antiquark being also a color antitriplet state $\overline{3}$, their product is decomposed into two irreducible representations, $\overline{3} \times \overline{3}=3+\overline{6}$, from which the color triplet 3 is kept. This color-triplet triquark makes a color-singlet bound state with the light diquark through the second flux tube in much the same way as the quark and diquark bind in an ordinary baryon. This approach differs from the one [51, 52] used previously to get the mass spectrum of the $S$ - and $P$-wave pentaquark states with the spin-parities $J^{P}=1 / 2^{ \pm}, 3 / 2^{-}$, and $5 / 2^{ \pm}$, where the two diquarks are assumed to be organized first into a color-triplet tetraquark state which afterwards interacts with the charm antiquark.

The double-charm triquark system $[[c q] \bar{c}]_{3}$ is expected to be dynamically similar to the double-charm antidiquark, $\{\bar{c} \bar{c}\}_{3}$, as far as their color and masses are concerned. The role of the latter, forming an almost static source, has been discussed at great length in a number of papers for the doubly-heavy anti-baryons, such as $\bar{\Xi}_{c c}(=\bar{c} \bar{c} \bar{q})$, and tetraquarks $T\left(\bar{c} \bar{c} q q^{\prime}\right)[38,41-46]$. The doubly-heavy triquark and the doubly-heavy diquark also differ in that the former has a light quark and the latter none. However, under the assumption 


\begin{tabular}{|l|l|}
\hline$J^{P}$ & $\left|S_{h d}, S_{t}, L_{t} ; S_{l d}, L_{l d} ; S, L\right\rangle_{J}$ \\
\hline \hline $1 / 2^{-}$ & $|0,1 / 2,0 ; 0,0 ; 1 / 2,0\rangle_{1 / 2}$ \\
\hline $1 / 2^{-}$ & $|1,1 / 2,0 ; 0,0 ; 1 / 2,0\rangle_{1 / 2}$ \\
$3 / 2^{-}$ & $|1,3 / 2,0 ; 0,0 ; 3 / 2,0\rangle_{3 / 2}$ \\
\hline
\end{tabular}

Table 2. Spin-parity $J^{P}$ and state vectors of the $S$-wave pentaquarks containing the "good" light diquark with the spin $S_{l d}=0$. The horizontal line demarcates the spin $S_{h d}$ of the heavy diquark.

\begin{tabular}{|l|l|}
\hline$J^{P}$ & $\left|S_{h d}, S_{t}, L_{t} ; S_{l d}, L_{l d} ; S, L\right\rangle_{J}$ \\
\hline \hline $1 / 2^{-}$ & $|0,1 / 2,0 ; 1,0 ; 1 / 2,0\rangle_{1 / 2}$ \\
$3 / 2^{-}$ & $|0,1 / 2,0 ; 1,0 ; 3 / 2,0\rangle_{3 / 2}$ \\
\hline $1 / 2^{-}$ & $|1,1 / 2,0 ; 1,0 ; 1 / 2,0\rangle_{1 / 2}$ \\
$3 / 2^{-}$ & $|1,1 / 2,0 ; 1,0 ; 3 / 2,0\rangle_{3 / 2}$ \\
$1 / 2^{-}$ & $|1,3 / 2,0 ; 1,0 ; 1 / 2,0\rangle_{1 / 2}$ \\
$3 / 2^{-}$ & $|1,3 / 2,0 ; 1,0 ; 3 / 2,0\rangle_{3 / 2}$ \\
$5 / 2^{-}$ & $|1,3 / 2,0 ; 1,0 ; 5 / 2,0\rangle_{5 / 2}$ \\
\hline
\end{tabular}

Table 3. Spin-parity $J^{P}$ and state vectors of the $S$-wave pentaquarks containing the "bad" light diquark with the spin $S_{l d}=1$. The horizontal line demarcates the spin $S_{h d}$ of the heavy diquark.

that the diquark $[c q]_{3}$ is bound, and the dynamics is essentially determined by the color configuration and masses, the two are expected not to deviate from each other, at least as a first approximation. With this in mind, we first detail the doubly-heavy triquark - light diquark picture, modify the effective Hamiltonian for the $S$-wave pentaquarks $[51,52]$ by keeping the most relevant terms for the mass determination, and then extend it for the $P$ states by including the orbital, spin-orbit and tensor interactions between the hidden-charm triquark and light diquark.

The effective Hamiltonian for the ground-state pentaquarks is described in terms of two constituent diquark masses of the heavy diquark $m_{[c q]} \equiv m_{h d}$ and of the light one $m_{\left[q^{\prime} q^{\prime \prime}\right]} \equiv$ $m_{l d}$, the spin-spin interactions between the quarks in each diquark shell, and spin-spin interactions between the diquarks. To these are added the constituent mass $m_{c}$ of the charm antiquark and its spin-spin interactions with each of the diquarks.

Using the quantum numbers introduced above, we specify the complete orthogonal set of basis vectors for the hidden-charm pentaquark states. As already stated, we define the state vectors having a total angular momentum quantum number $J$ by

$$
\left|S_{h d}, S_{t}, L_{t} ; S_{l d}, L_{l d} ; S, L\right\rangle_{J}
$$

The corresponding sets of the $S$-wave state vectors (with $L_{t}=L_{l d}=L=0$ ) with the "good" $\left(S_{l d}=0\right)$ and "bad" $\left(S_{l d}=1\right)$ light diquark are presented in tables 2 and 3 , respectively. Among the overall ten states with a fixed light-quark flavor content, there are five states with $J^{P}=1 / 2^{-}$, four states with $J^{P}=3 / 2^{-}$and one with $J^{P}=5 / 2^{-}$. In view of the propensity of these states, we note that in the heavy-quark symmetry limit, the production of the states with the quantum numbers shown in table 3 is forbidden in $\Lambda_{b^{-}}$or $\Xi_{b^{-}}$-decays. Nevertheless, they are present in the pentaquark mass spectrum, and they are reachable in other processes. 


\begin{tabular}{|l|l|}
\hline$J^{P}$ & $\left|S_{h d}, S_{t}, L_{t} ; S_{l d}, L_{l d} ; S, L\right\rangle_{J}$ \\
\hline \hline $1 / 2^{+}$ & $|0,1 / 2,0 ; 0,1 ; 1 / 2,1\rangle_{1 / 2}$ \\
$3 / 2^{+}$ & $|0,1 / 2,0 ; 0,1 ; 1 / 2,1\rangle_{3 / 2}$ \\
\hline $1 / 2^{+}$ & $|1,1 / 2,0 ; 0,1 ; 1 / 2,1\rangle_{1 / 2}$ \\
$3 / 2^{+}$ & $|1,1 / 2,0 ; 0,1 ; 1 / 2,1\rangle_{3 / 2}$ \\
$1 / 2^{+}$ & $|1,3 / 2,0 ; 0,1 ; 3 / 2,1\rangle_{1 / 2}$ \\
$3 / 2^{+}$ & $|1,3 / 2,0 ; 0,1 ; 3 / 2,1\rangle_{3 / 2}$ \\
$5 / 2^{+}$ & $|1,3 / 2,0 ; 0,1 ; 3 / 2,1\rangle_{5 / 2}$ \\
\hline
\end{tabular}

Table 4. Spin-parity $J^{P}$ and state vectors of the $P$-wave pentaquarks with the groundstate triquark $\left(L_{t}=0\right)$ and "good" light diquark with the spin $S_{l d}=0$. The horizontal line demarcates the spin $S_{h d}$ of the heavy diquark.

\begin{tabular}{|l|l|}
\hline$J^{P}$ & $\left|S_{h d}, S_{t}, L_{t} ; S_{l d}, L_{l d} ; S, L\right\rangle_{J}$ \\
\hline \hline $1 / 2^{+}$ & $|0,1 / 2,0 ; 1,1 ; 1 / 2,1\rangle_{1 / 2}$ \\
$3 / 2^{+}$ & $|0,1 / 2,0 ; 1,1 ; 1 / 2,1\rangle_{3 / 2}$ \\
$1 / 2^{+}$ & $|0,1 / 2,0 ; 1,1 ; 3 / 2,1\rangle_{1 / 2}$ \\
$3 / 2^{+}$ & $|0,1 / 2,0 ; 1,1 ; 3 / 2,1\rangle_{3 / 2}$ \\
$5 / 2^{+}$ & $|0,1 / 2,0 ; 1,1 ; 3 / 2,1\rangle_{5 / 2}$ \\
\hline $1 / 2^{+}$ & $|1,1 / 2,0 ; 1,1 ; 1 / 2,1\rangle_{1 / 2}$ \\
$3 / 2^{+}$ & $|1,1 / 2,0 ; 1,1 ; 1 / 2,1\rangle_{3 / 2}$ \\
$1 / 2^{+}$ & $|1,1 / 2,0 ; 1,1 ; 3 / 2,1\rangle_{1 / 2}$ \\
$3 / 2^{+}$ & $|1,1 / 2,0 ; 1,1 ; 3 / 2,1\rangle_{3 / 2}$ \\
$5 / 2^{+}$ & $|1,1 / 2,0 ; 1,1 ; 3 / 2,1\rangle_{5 / 2}$ \\
$1 / 2^{+}$ & $|1,3 / 2,0 ; 1,1 ; 1 / 2,1\rangle_{1 / 2}$ \\
$3 / 2^{+}$ & $|1,3 / 2,0 ; 1,1 ; 1 / 2,1\rangle_{3 / 2}$ \\
$1 / 2^{+}$ & $|1,3 / 2,0 ; 1,1 ; 3 / 2,1\rangle_{1 / 2}$ \\
$3 / 2^{+}$ & $|1,3 / 2,0 ; 1,1 ; 3 / 2,1\rangle_{3 / 2}$ \\
$5 / 2^{+}$ & $|1,3 / 2,0 ; 1,1 ; 3 / 2,1\rangle_{5 / 2}$ \\
$3 / 2^{+}$ & $|1,3 / 2,0 ; 1,1 ; 5 / 2,1\rangle_{3 / 2}$ \\
$5 / 2^{+}$ & $|1,3 / 2,0 ; 1,1 ; 5 / 2,1\rangle_{5 / 2}$ \\
$7 / 2^{+}$ & $|1,3 / 2,0 ; 1,1 ; 5 / 2,1\rangle_{7 / 2}$ \\
\hline
\end{tabular}

Table 5. Spin-parity $J^{P}$ and state vectors of the $P$-wave pentaquarks with the groundstate triquark $\left(L_{t}=0\right)$ and "bad" light diquark with the spin $S_{l d}=1$. The horizontal line demarcates the spin $S_{h d}$ of the heavy diquark.

For the orbitally excited states, one needs to specify which part of the pentaquark wave function is excited. In the triquark-diquark template used here, and shown in figure 1, the heavy triquark state consists of the charm diquark and charm antiquark. Since both are heavy, the triquark is an (almost) static system and, hence, $L_{t}=0$ is its most probable orbital quantum number. Thus, the orbital excitation is generated by the light diquark (i.e., $\left.L=L_{l d}\right)$. With this, we list the lowest-lying orbitally excited states $(L=1)$ with the "good" $\left(S_{l d}=0\right)$ and the "bad" $\left(S_{l d}=1\right)$ light diquark in tables 4 and 5 , respectively. In total, there are 25 pentaquark states with a fixed light-quark flavor content which are divided into nine states with $J^{P}=1 / 2^{+}$, ten with $J^{P}=3 / 2^{+}$, five with $J^{P}=5 / 2^{+}$and one with $J^{P}=7 / 2^{+}$.

The pentaquark spectrum emerging from the underlying diquark picture is very rich. Contrasting it with the current experimental situation, with only three known hidden- 
charm pentaquark states $P_{c}(4312)^{ \pm}, P_{c}(4440)^{ \pm}$, and $P_{c}(4457)^{ \pm}$observed in $\Lambda_{b}$-baryon decays, some selection rules have to be applied to restrict the number of observable pentaquarks. Since in the heavy-quark symmetry limit, the spin of the light quarks is conserved in heavy-baryon decays, and $S_{l d}=0$ in $\Lambda_{b}$-baryon, the states with the light-diquark spin $S_{l d}=1$ are suppressed. This means that we need to consider only the states in tables 2 and 4, for the $S$ - and $P$-wave pentaquarks, respectively. This reduces the number of states to three ( $S$-wave) and seven ( $P$-wave). The states with $S_{l d}=1$ can, however, be produced in $\Omega_{b}$-baryon decays or in prompt production processes.

A related issue is the presence of two identical light quarks in the Fock space of a hidden-charm pentaquark. Among the light quark $q$ present in the triquark, and the light quarks $q^{\prime}$ and $q^{\prime \prime}$ in the diquark, there are two identical quarks. Treated as free quarks, they are subject to Pauli's exclusion principle, affecting the resulting spectrum of hadrons. This, for example, would be the case in the hadroquarkonium picture of the hidden-charm pentaquarks. However, if the $q^{\prime} q^{\prime \prime}$-pair is bound in a diquark, which is a boson, then this exclusion does not apply so long as the diquark is not broken into its fermionic constituents. We illustrate this situation by focusing on the discovery mode $\Lambda_{b} \rightarrow P_{c}^{+} K^{-}$. This decay is induced at the quark level by the $b[u d] \rightarrow c \bar{c} s[u d]+(u \bar{u})_{\text {vac }}$ transition. In this, the $[u d]$-diquark, which has well-defined quantum numbers ( $\operatorname{spin} S_{[u d]}=$ 0 and isospin $I_{[u d]}=0$ ), is a boson. In the heavy-quark symmetry limit, assumed in our paper, the light degrees of freedom remain conserved, and the light $[u d]$-diquark emerges in tact from the decay. Indeed, this imposes restrictions on the quantum numbers of final states, as we have emphasized earlier. If this symmetry is broken, say, by $1 / m_{b}$ terms, then the $u$ - and $d$-quarks in the $[u d]$-diquark are no longer bound, and in the example here, one has two identical quarks, the $u$-quark produced from the vacuum excitation and the $u$-quark from the break-up of the spectator diquark, and the Pauli's exclusion principle would apply. In the heavy-quark symmetry limit, we have the spin- $1 / 2 u$-quark from the vacuum excitation, and the spin-0 $[u d]$-diquark, and they are distinctly different building blocks. In this case, no exclusion restrictions from identical quarks apply, which is what we have used in building the pentaquark spectrum.

\section{Effective Hamiltonian for pentaquark spectrum}

We calculate the mass spectrum of pentaquark under the assumption that their underlying structure is given by $\bar{c},[c q]$, and $\left[q^{\prime} q^{\prime \prime}\right]$, with $q, q^{\prime}$, and $q^{\prime \prime}$ being any of the light $u-, d-$, and $s$-quarks. We also assume the isospin symmetry among the states. For this, we extend the effective Hamiltonian proposed for the tetraquark spectroscopy by Maiani et al. [56]. The effective Hamiltonian for the $S$-wave pentaquark mass spectrum can be written as follows:

$$
H^{(L=0)}=H_{t}+H_{l d}
$$

The first term in the Hamiltonian (3.1) is related with the color triquark:

$$
H_{t}=m_{c}+m_{h d}+2\left(\mathcal{K}_{c q}\right)_{\overline{3}}\left(\mathbf{S}_{c} \cdot \mathbf{S}_{q}\right)+2 \mathcal{K}_{\bar{c} q}\left(\mathbf{S}_{\bar{c}} \cdot \mathbf{S}_{q}\right)+2 \mathcal{K}_{\bar{c} c}\left(\mathbf{S}_{\bar{c}} \cdot \mathbf{S}_{c}\right)
$$


where $m_{c}$ and $m_{h d}$ are the constituent masses of the charm antiquark and charm diquark, respectively. The last three terms describe the spin-spin interactions in the charm diquark and between the diquark constituents and the charm antiquark. Among the three spin-spin couplings $\left(\mathcal{K}_{c q}\right)_{\overline{3}}, \mathcal{K}_{\bar{c} q}$, and $\mathcal{K}_{\bar{c} c}$, the spin-spin interaction inside the diquark $\left(\mathcal{K}_{c q}\right) \overline{3}$ is argued to be the dominant one [56].

The second term $H_{l d}$ in the Hamiltonian (3.1) contains the operators responsible for the spin-spin interaction in the light diquark and its interaction with the triquark:

$$
H_{l d}=m_{l d}+2\left(\mathcal{K}_{q^{\prime} q^{\prime \prime}}\right)_{\overline{3}}\left(\mathbf{S}_{q^{\prime}} \cdot \mathbf{S}_{q^{\prime \prime}}\right)+H_{S S}^{t-l d},
$$

where $m_{l d}$ is the constituent mass of the light diquark, consisting of the light quarks $q^{\prime}$ and $q^{\prime \prime}$, and the contribution of the spin-spin interaction in this diquark to the pentaquark mass is determined by the coupling $\left(\mathcal{K}_{q^{\prime} q^{\prime \prime}}\right)_{\overline{3}}$. The last term in $H_{l d}$ is responsible for all the possible spin-spin interactions between the constituents of the light diquark and doublyheavy triquark:

$$
\begin{aligned}
H_{S S}^{t-l d}= & 2\left(\tilde{\mathcal{K}}_{c q^{\prime}}\right) \overline{3}\left(\mathbf{S}_{c} \cdot \mathbf{S}_{q^{\prime}}\right)+2\left(\tilde{\mathcal{K}}_{q q^{\prime}}\right) \overline{3}\left(\mathbf{S}_{q} \cdot \mathbf{S}_{q^{\prime}}\right)+2 \tilde{\mathcal{K}}_{\bar{c} q^{\prime}}\left(\mathbf{S}_{\bar{c}} \cdot \mathbf{S}_{q^{\prime}}\right) \\
& +2\left(\tilde{\mathcal{K}}_{c q^{\prime \prime}}\right) \overline{3}\left(\mathbf{S}_{c} \cdot \mathbf{S}_{q^{\prime \prime}}\right)+2\left(\tilde{\mathcal{K}}_{q q^{\prime \prime}}\right) \overline{3}\left(\mathbf{S}_{q} \cdot \mathbf{S}_{q^{\prime \prime}}\right)+2 \tilde{\mathcal{K}}_{\bar{c} q^{\prime \prime}}\left(\mathbf{S}_{\bar{c}} \cdot \mathbf{S}_{q^{\prime \prime}}\right) .
\end{aligned}
$$

Here, the coefficients with the tilde differ from the ones introduced above as the former show the strengths of the spin-spin interactions inside the compact objects like diquarks and triquark while the later ones determine the strengths between the constituents of two objects, the heavy triquark and light diquark, and are strongly suppressed. The rationale of this is specific to the diquark model, in which the hadronic size of the diquarks is much smaller or compared to the overall size of the multiquark hadrons. Thus, only local spinspin interactions (within a diquark or triquark) are allowed. This then accounts for all possible spin-spin interactions and completes the content of the effective Hamiltonian (3.1) for the masses of the ground-state pentaquarks. A comment is in order: Despite the fact that this picture, which is physically more motivated, differs form the one used in [51, 52], for the analysis and predictions of the ground-state pentaquarks, actually all the terms in the effective Hamiltonian (3.1) are the same.

The general form of the effective Hamiltonian for the orbitally-excited pentaquark mass spectrum can be written as follows:

$$
H=H^{(L=0)}+H_{L}+H_{T} .
$$

In addition to the spin-spin interactions introduced for the ground-state pentaquarks described above, the extended effective Hamiltonian (3.5) includes terms explicitly dependent on the internal orbital angular momentum $L_{t}$ of the hidden-charm triquark and orbital momentum $L_{l d}$ of the light diquark relative to the triquark system. The corresponding Hamiltonian is called $H_{L}$ in (3.5). The terms relevant for the tensor interactions in each subsystem specified above are subsumed in $H_{T}$ in (3.5). As already stated, we assume that the triquark state is an $S$-wave (i.e., $L_{t}=0$ ). Thus, $L=L_{l d}$. Inclusion of the triquark orbital angular momentum $L_{t}$ will be commented later on. The terms in $H_{L}$ which contain 
the orbital angular momentum operator $\mathbf{L}$ are as follows:

$$
H_{L}=2 A_{t}\left(\mathbf{S}_{t} \cdot \mathbf{L}\right)+2 A_{l d}\left(\mathbf{S}_{l d} \cdot \mathbf{L}\right)+\frac{1}{2} B \mathbf{L}^{2},
$$

where quantities $A_{t}, A_{l d}$, and $B$ parametrize the strengths of the triquark spin-orbit, lightdiquark spin-orbit and orbital momentum couplings, respectively. The last term in (3.5) represents the tensor interaction among the heavy triquark and light diquark:

$$
H_{T}=b\left[3 \frac{\left(\mathbf{S}_{t} \cdot \mathbf{R}\right)\left(\mathbf{S}_{l d} \cdot \mathbf{R}\right)}{R^{2}}-\left(\mathbf{S}_{t} \cdot \mathbf{S}_{l d}\right)\right],
$$

where $\mathbf{R}$ determines the position of the light diquark relative to the heavy triquark and $b$ is the strength of the tensor interaction.

The inclusion of a possible excitation in the triquark system $\left(L_{t} \neq 0\right)$ results into additional terms in the effective Hamiltonian:

$$
\Delta H=\Delta H_{L}+\Delta H_{T} .
$$

The structure of $\Delta H_{L}$ which includes the angular momentum operator $\mathbf{L}_{t}$ in the triquark system is similar to $(3.6)$ :

$$
\Delta H_{L}=2 A_{\bar{c}}\left(\mathbf{S}_{\bar{c}} \cdot \mathbf{L}_{t}\right)+2 A_{h d}\left(\mathbf{S}_{h d} \cdot \mathbf{L}_{t}\right)+\frac{1}{2} B_{t} \mathbf{L}_{t}^{2},
$$

where quantities $A_{\bar{c}}, A_{h d}$, and $B_{t}$ parametrize the strengths of the charm antiquark and diquark spin-orbit and triquark orbital momentum couplings, respectively. As the triquark contains charm quark and antiquark, one expects a suppression of $A_{\bar{c}}, A_{h d}$, and $B_{t}$ by the $c$-quark mass in comparison with the parameters $A_{t}, A_{l d}$, and $B$ entering (3.6). In the numerical calculations, we set them to zero.

The second term in (3.8) represents the tensor interaction among the charm diquark and antiquark in the triquark:

$$
\Delta H_{T}=b_{t}\left[3 \frac{\left(\mathbf{S}_{\bar{c}} \cdot \mathbf{r}\right)\left(\mathbf{S}_{h d} \cdot \mathbf{r}\right)}{r^{2}}-\left(\mathbf{S}_{\bar{c}} \cdot \mathbf{S}_{h d}\right)\right],
$$

where $\mathbf{r}$ determines the position of the charm diquark relative to the charm antiquark and $b_{t}$ is the strength of this interaction. In the phenomenological analysis of the hiddencharm orbitally excited $Y$-tetraquarks, it was shown [57] that a similar strength entering the tensor interaction between the charm diquark and antidiquark is consistent with zero. Following [57], it is reasonable to assume that $b_{t}$ in (3.10) is also small, and hence we neglect this interaction in the analysis reported below by setting $\Delta H_{T}=0$.

Returning to the discussion of the tensor contribution, the expression in eq. (3.7) is of the following general form:

$$
Q\left(\mathbf{S}_{1}, \mathbf{S}_{2}\right)=3\left(\mathbf{S}_{1} \cdot \mathbf{n}\right)\left(\mathbf{S}_{2} \cdot \mathbf{n}\right)-\left(\mathbf{S}_{1} \cdot \mathbf{S}_{2}\right)=3 S_{1 i} S_{2 j} N_{i j},
$$

where $\mathbf{S}_{1}$ and $\mathbf{S}_{2}$ are the spins of two particles and $\mathbf{n}=\mathbf{R} / R$ is the unit vector in the direction of $\mathbf{R}$. This notation was used by us previously [57] in the analysis of the orbitally 
excited $(L=1) \Omega_{c}$-baryons and $Y$-tetraquarks. In both cases $\mathbf{S}_{1}$ was identified with the spin of the diquark, $\mathbf{S}_{Q}$, and $\mathbf{S}_{2}$ is either the spin of the charm quark, $\mathbf{S}_{c}$, in the $\Omega_{c}$-baryon, or the spin of the antidiquark, $\mathbf{S}_{\bar{Q}}$, in the $Y$-tetraquark.

The scalar operator $Q\left(\mathbf{S}_{1}, \mathbf{S}_{2}\right)$ in eq. (3.11) is expressed as the convolution of spins with the tensor operator:

$$
N_{i j}=n_{i} n_{j}-\frac{1}{3} \delta_{i j}
$$

For further applications, we need the matrix elements of the operator $N_{i j}$ between the states with the same fixed value $L$ of the orbital angular momentum operator $\mathbf{L}$ which can be obtained with the help of the identity from Landau and Lifshitz [58]:

$$
\left\langle N_{i j}\right\rangle=-\frac{1}{(2 L-1)(2 L+3)}\left[L_{i} L_{j}+L_{j} L_{i}-\frac{2}{3} \delta_{i j} L(L+1)\right] .
$$

It is obvious that this matrix element is trivial when the two-particle quantum state is in the $S$-wave, $L=0$.

In terms of $Q\left(\mathbf{S}_{1}, \mathbf{S}_{2}\right)$, eq. (3.7) for $L=1$ can be expressed as follows

$$
H_{T}=b\left\langle Q\left(\mathbf{S}_{t}, \mathbf{S}_{l d}\right)\right\rangle=-\frac{3}{5} b\left\langle\left(\mathbf{L} \cdot \mathbf{S}_{t}\right)\left(\mathbf{L} \cdot \mathbf{S}_{l d}\right)+\left(\mathbf{L} \cdot \mathbf{S}_{l d}\right)\left(\mathbf{L} \cdot \mathbf{S}_{t}\right)-\frac{4}{3}\left(\mathbf{S}_{t} \cdot \mathbf{S}_{l d}\right)\right\rangle,
$$

and similarly $\Delta H_{T}=b_{t} Q\left(\mathbf{S}_{h d}, \mathbf{S}_{\bar{c}}\right)$, if $b_{t} \neq 0$. In eq. (3.14), the mixed-spin $Q$-operator can be reexpressed in terms of the single spin ones:

$$
Q\left(\mathbf{S}_{t}, \mathbf{S}_{l d}\right)=Q\left(\mathbf{S}_{l d}, \mathbf{S}_{t}\right)=\frac{1}{2}\left[Q(\mathbf{S}, \mathbf{S})-Q\left(\mathbf{S}_{t}, \mathbf{S}_{t}\right)-Q\left(\mathbf{S}_{l d}, \mathbf{S}_{l d}\right)\right],
$$

where $\mathbf{S}=\mathbf{S}_{t}+\mathbf{S}_{l d}$. To calculate the single-spin $Q$-operators inside the brackets, it is necessary to account for appropriate commutation relations of the components of $\mathbf{L}$ and $\mathbf{S}_{X}$, where $\mathbf{S}_{X}=\mathbf{S}_{t}, \mathbf{S}_{l d}$, and $\mathbf{S}$ are the spins of the triquark, light-diquark, and the total spin of the pentaquark, respectively. Setting $L=1$, one finds easily [59]:

$$
\left\langle Q\left(\mathbf{S}_{X}, \mathbf{S}_{X}\right)\right\rangle=-\frac{3}{5}\left\langle 2\left(\mathbf{L} \cdot \mathbf{S}_{X}\right)^{2}+\left(\mathbf{L} \cdot \mathbf{S}_{X}\right)-\frac{4}{3}\left(\mathbf{S}_{X} \cdot \mathbf{S}_{X}\right)\right\rangle .
$$

The matrix elements can be computed directly by applying the operators $\left(\mathbf{L} \cdot \mathbf{S}_{X}\right)$ to the products of states corresponding to the individual spins and angular momenta. More effectively, one can use Wigner $6 j$-symbols, as is customary for analogous cases in atomic and nuclear physics. An explicit example of their application to the analysis of the orbitally-excited $\Omega_{c}$-baryons and $Y$-tetraquarks is presented in the appendix of ref. [57]. So, to get the mass predictions one needs to specify a concrete basis of the orbitally-excited pentaquark states in which the mass predictions can be done.

\section{Mass formulae for pentaquark spectrum}

\section{1 $S$-wave pentaquarks}

With the basis vectors of the pentaquark states chosen, one can derive analytical expressions for calculating the pentaquark spectrum. They are the matrix elements of the effective 
Hamiltonian presented above in section 3. Note that this is simpler for the Model II by Maiani et al. [56], but becomes more involved in the Model I [35], where additional mixings between the spins of (anti)quarks in compact shells are included. As the later couplings are suppressed in comparison with the spin-spin interactions inside the shells, we neglect the later mixings and restrict ourselves with the Model II.

Restricting ourselves to the light $u$ - and $d$-quarks, and assuming isospin symmetry, the universal contribution entering all the pentaquark states is defined as $M_{0}$, which is the sum of the constituent masses of the heavy and light diquarks and charm antiquark:

$$
M_{0} \equiv m_{h d}+m_{l d}+m_{c} .
$$

Apart from this, there are two terms in the effective Hamiltonian explicitly related with the spins of the diquarks [see eqs. (3.2) and (3.3)]:

$$
\begin{gathered}
{ }_{J}\left\langle S_{h d}, S_{t}, L_{t} ; S_{l d}, L_{l d} ; S, L\left|2\left(\mathcal{K}_{c q}\right)_{\overline{3}}\left(\mathbf{S}_{c} \cdot \mathbf{S}_{q}\right)\right| S_{h d}, S_{t}, L_{t} ; S_{l d}, L_{l d} ; S, L\right\rangle_{J} \\
=\left(\mathcal{K}_{c q}\right)_{\overline{3}}\left[S_{h d}\left(S_{h d}+1\right)-\frac{3}{2}\right]=\frac{1}{2}\left(\mathcal{K}_{c q}\right)_{\overline{3}} \times\left\{\begin{array}{c}
-3, S_{h d}=0, \\
1, \quad S_{h d}=1,
\end{array}\right. \\
{ }_{J}\left\langle S_{h d}, S_{t}, L_{t} ; S_{l d}, L_{l d} ; S, L\left|2\left(\mathcal{K}_{q^{\prime} q^{\prime \prime}}\right)_{\overline{3}}\left(\mathbf{S}_{q^{\prime}} \cdot \mathbf{S}_{q^{\prime \prime}}\right)\right| S_{h d}, S_{t}, L_{t} ; S_{l d}, L_{l d} ; S, L\right\rangle_{J} \\
=\left(\mathcal{K}_{q^{\prime} q^{\prime \prime}}\right)_{\overline{3}}\left[S_{l d}\left(S_{l d}+1\right)-\frac{3}{2}\right]=\frac{1}{2}\left(\mathcal{K}_{q^{\prime} q^{\prime \prime}}\right)_{\overline{3}} \times\left\{\begin{array}{c}
-3, S_{l d}=0, \\
1,
\end{array} S_{l d}=1 .\right.
\end{gathered}
$$

The two terms left in eq. (3.2) are responsible for the contributions of the spin-spin interactions between the charm antiquark and the quarks inside the heavy diquark, which together form the triquark state. To calculate their impact, it is convenient to use the Wigner $6 j$ symbols, which allows us to describe a recoupling between three angular momenta, say $j_{1}$, $j_{2}$ and $j_{3}$, which are combined into the state with the total momentum $J$. Following the angular momentum sum rules in quantum mechanics, one combines two of the three momenta first and then adds the third momentum. Thus, there are three possibilities: in the the first, the momenta $j_{1}$ and $j_{2}$ couple together in the state with the angular momentum $j_{12}$, which after combining with $j_{3}$ results in the state with total angular momentum $J$; in the second, the momenta $j_{1}$ and $j_{3}$ produce the state with the momentum $j_{13}$ and it is combined with $j_{2}$ to get the state with the momentum $J$; and in the third, the momenta $j_{2}$ and $j_{3}$ produce the state with the momentum $j_{23}$ which then combines with $j_{1}$ to obtain the state with the momentum $J$. Each way of summing up the momenta results in its own set of basis vectors. If, for example, the momentum $j_{2}$ is coupled initially to $j_{1}$ and one wants to recouple it to $j_{3}$, there are two extra momenta $j_{12}$ and $j_{23}$, each of which has its own set of basis vectors, $\left|\left(j_{1}, j_{2}\right)_{j_{12}}, j_{3} ; J\right\rangle$ and $\left|j_{1},\left(j_{2}, j_{3}\right)_{j_{23}} ; J\right\rangle$. The Wigner $6 j$-symbols describe the transformation from one basis to the other as follows [60]:

$$
\left|j_{1},\left(j_{2}, j_{3}\right)_{j_{23}} ; J\right\rangle=\sum_{j_{12}}(-1)^{j_{1}+j_{2}+j_{3}+J} \sqrt{\left(2 j_{12}+1\right)\left(2 j_{23}+1\right)}\left\{\begin{array}{ccc}
j_{1} & j_{2} & j_{12} \\
j_{3} & J & j_{23}
\end{array}\right\}\left|\left(j_{1}, j_{2}\right)_{j_{12}}, j_{3} ; J\right\rangle .
$$

Here, Wigner $6 j$-symbols are represented by the curly brackets. To find the matrix elements of the spin-spin operators $2 \mathcal{K}_{\bar{c} q}\left(\mathbf{S}_{\bar{c}} \cdot \mathbf{S}_{q}\right)$ and $2 \mathcal{K}_{\bar{c} c}\left(\mathbf{S}_{\bar{c}} \cdot \mathbf{S}_{c}\right)$ in eq. (3.2) with 
the help of the Wigner $6 j$-symbols, it is convenient to rewrite the pentaquark vector state $\left|S_{h d}, S_{t}, L_{t} ; S_{l d}, L_{l d} ; S, L\right\rangle_{J}$ as $\left|S_{\bar{c}},\left(S_{q}, S_{c}\right)_{S_{h d}} ; S_{t}\right\rangle$ and $\left|S_{\bar{c}},\left(S_{c}, S_{q}\right)_{S_{h d}} ; S_{t}\right\rangle$, respectively, (here, all the unnecessary fixed quantum numbers are omitted from the vector and the charm antiquark spin, $S_{\bar{c}}$, is shown explicitly) and make the angular momenta recoupling as in eq. (4.4):

$$
\begin{aligned}
\left|S_{\bar{c}},\left(S_{q}, S_{c}\right)_{S_{h d}} ; S_{t}\right\rangle= & \sum_{S_{\bar{c} q}}(-1)^{S_{\bar{c}}+S_{q}+S_{c}+S_{t}} \sqrt{\left(2 S_{\bar{c} q}+1\right)\left(2 S_{h d}+1\right)} \\
& \times\left\{\begin{array}{l}
S_{\bar{c}} S_{q} S_{\bar{c} q} \\
S_{c} S_{t} S_{h d}
\end{array}\right\}\left|\left(S_{\bar{c}}, S_{q}\right)_{S_{\bar{c} q}}, S_{c} ; S_{t}\right\rangle, \\
\left|S_{\bar{c}},\left(S_{c}, S_{q}\right)_{S_{h d}} ; S_{t}\right\rangle= & \sum_{S_{\bar{c} c}}(-1)^{S_{\bar{c}}+S_{q}+S_{c}+S_{t}} \sqrt{\left(2 S_{\bar{c} c}+1\right)\left(2 S_{h d}+1\right)} \\
& \times\left\{\begin{array}{l}
S_{\bar{c}} S_{c} S_{\bar{c} c} \\
S_{q} S_{t} S_{h d}
\end{array}\right\}\left|\left(S_{\bar{c}}, S_{c}\right)_{S_{\bar{c} c}}, S_{q} ; S_{t}\right\rangle .
\end{aligned}
$$

The required matrix elements can be written in the form:

$$
\begin{aligned}
& J\left\langle S_{h d}^{\prime}, S_{t}, L_{t} ; S_{l d}, L_{l d} ; S, L\left|2 \mathcal{K}_{\bar{c} q}\left(\mathbf{S}_{\bar{c}} \cdot \mathbf{S}_{q}\right)\right| S_{h d}, S_{t}, L_{t} ; S_{l d}, L_{l d} ; S, L\right\rangle_{J} \\
&= \mathcal{K}_{\bar{c} q}(-1)^{2 S_{t}+1} \sqrt{\left(2 S_{h d}+1\right)\left(2 S_{h d}^{\prime}+1\right)} \sum_{S_{\bar{c} q}}\left(2 S_{\bar{c} q}+1\right)\left[S_{\bar{c} q}\left(S_{\bar{c} q}+1\right)-\frac{3}{2}\right] \\
& \times\left\{\begin{array}{rrr}
1 / 2 & 1 / 2 & S_{\bar{c} q} \\
1 / 2 & S_{t} & S_{h d}
\end{array}\right\}\left\{\begin{array}{rrr}
1 / 2 & 1 / 2 & S_{\bar{c} q} \\
1 / 2 & S_{t} & S_{h d}^{\prime}
\end{array}\right\}, \\
& J\left\langle S_{h d}^{\prime}, S_{t}, L_{t} ; S_{l d}, L_{l d} ; S, L\left|2 \mathcal{K}_{\bar{c} c}\left(\mathbf{S}_{\bar{c}} \cdot \mathbf{S}_{c}\right)\right| S_{h d}, S_{t}, L_{t} ; S_{l d}, L_{l d} ; S, L\right\rangle_{J} \\
&=\mathcal{K}_{\bar{c} c}(-1)^{2 S_{t}+1} \sqrt{\left(2 S_{h d}+1\right)\left(2 S_{h d}^{\prime}+1\right)} \sum_{S_{\bar{c} c}}\left(2 S_{\bar{c} c}+1\right)\left[S_{\bar{c} c}\left(S_{\bar{c} c}+1\right)-\frac{3}{2}\right] \\
& \quad \times\left\{\begin{array}{lll}
1 / 2 & 1 / 2 & S_{\bar{c} c} \\
1 / 2 & S_{t} & S_{h d}
\end{array}\right\}\left\{\begin{array}{lll}
1 / 2 & 1 / 2 & S_{\bar{c} c} \\
1 / 2 & S_{t} & S_{h d}^{\prime}
\end{array}\right\},
\end{aligned}
$$

where spins of all the quarks are replaced by their numerical values, $S_{\bar{c}}=S_{c}=S_{q}=1 / 2$. Up to the factors $\mathcal{K}_{\bar{c} q}$ and $\mathcal{K}_{\bar{c} c}$, both equations coincide, so the contribution of these two terms entering the effective Hamiltonian to the mass formulae is proportional to the sum of the coupling strengths, $\mathcal{K}_{\bar{c} q}+\mathcal{K}_{\bar{c} c}$.

The matrix elements of the operators in eq. (3.4) have in general six different coupling strengths. For the pentaquark having the light $u$ - and $d$-quarks only, isospin symmetry results into relations among the couplings. This simplifies the spin-spin interaction (3.4) substantially. For the pentaquarks having one or more strange quarks, some of the relations no longer hold, as $\mathrm{SU}(3)_{F}$-symmetry is broken. Details of the matrix element calculation in this case are presented in appendix A. This contribution will be neglected in the numerical analysis as we are working in the Model II [56].

We apply the above formalism for the calculation of the mass spectrum beginning from the pentaquark states from table 2. From three states presented there, two states 
with $J^{P}=1 / 2^{-}$mix due to the spin-spin interaction of the charm antiquark and the heavy diquark, and the third one with $J^{P}=3 / 2^{-}$remains unmixed. For the later state, the mass $m_{3}^{S 0}$ (here, the superscript denotes the $S$-wave pentaquark with the "good" light diquark, $\left.S_{l d}=0\right)$ is the average of the effective Hamiltonian over this state:

$$
m_{3}^{S 0}=M_{0}+\frac{1}{2}\left(\mathcal{K}_{c q}\right)_{\overline{3}}-\frac{3}{2}\left(\mathcal{K}_{q^{\prime} q^{\prime \prime}}\right)_{\overline{3}}+\frac{1}{2}\left(\mathcal{K}_{\bar{c} q}+\mathcal{K}_{\bar{c} c}\right),
$$

where $M_{0}$ is defined in (4.1). The former two states with $J^{P}=1 / 2^{-}$, after sandwiching the effective Hamiltonian, yield the following $(2 \times 2)$ mass matrix:

$$
M_{J=1 / 2}^{S 0}=M_{0}-\frac{1}{2}\left(\mathcal{K}_{c q}\right)_{\overline{3}}-\frac{3}{2}\left(\mathcal{K}_{q^{\prime} q^{\prime \prime}}\right)_{\overline{3}}-\left(\mathcal{K}_{c q}\right)_{\overline{3}}\left(\begin{array}{cc}
1 & 0 \\
0 & -1
\end{array}\right)+\frac{1}{2}\left(\mathcal{K}_{\bar{c} q}+\mathcal{K}_{\bar{c} c}\right)\left(\begin{array}{cc}
0 & \sqrt{3} \\
\sqrt{3} & -2
\end{array}\right) .
$$

Diagonalizing this matrix yields the masses of the other two states:

$$
\begin{aligned}
& m_{1}^{S 0}=M_{0}-\frac{1}{4}\left(\mathcal{K}_{\bar{c} q}+\mathcal{K}_{\bar{c} c}\right)\left[2+r_{h d}+3 r_{l d}+2 \sqrt{3+\left(1-r_{h d}\right)^{2}}\right] \\
& m_{2}^{S 0}=M_{0}-\frac{1}{4}\left(\mathcal{K}_{\bar{c} q}+\mathcal{K}_{\bar{c} c}\right)\left[2+r_{h d}+3 r_{l d}-2 \sqrt{3+\left(1-r_{h d}\right)^{2}}\right]
\end{aligned}
$$

where two ratios $r_{h d}$ and $r_{l d}$ of the couplings are defined as:

$$
r_{h d} \equiv \frac{2\left(\mathcal{K}_{c q}\right)_{\overline{3}}}{\mathcal{K}_{\bar{c} q}+\mathcal{K}_{\bar{c} c}}, \quad r_{l d} \equiv \frac{2\left(\mathcal{K}_{q^{\prime} q^{\prime \prime}}\right)_{\overline{3}}}{\mathcal{K}_{\bar{c} q}+\mathcal{K}_{\bar{c} c}}
$$

The averaged mass of the states with masses given in (4.11) and (4.12) has the following value:

$$
\bar{m}_{12}^{S 0}=\frac{1}{2}\left[m_{1}^{S 0}+m_{2}^{S 0}\right]=M_{0}-\frac{1}{4}\left(\mathcal{K}_{\bar{c} q}+\mathcal{K}_{\bar{c} c}\right)\left(2+r_{h d}+3 r_{l d}\right),
$$

and the mass of the third state lies above by an amount

$$
\Delta m_{3,12}^{S 0}=m_{3}^{S 0}-\bar{m}_{12}^{S 0}=\frac{1}{2}\left(\mathcal{K}_{\bar{c} q}+\mathcal{K}_{\bar{c} c}\right)\left(2+r_{h d}\right) .
$$

We continue calculations of the mass spectrum taking the $S$-wave pentaquark states with the "bad" light diquark from table 3. From the seven states presented there, two states with $J^{P}=1 / 2^{-}$and the other two with $J^{P}=3 / 2^{-}$, both pairs having the triquark spin $S_{t}=1 / 2$, mix due to the spin-spin interaction of the charm antiquark and the heavy diquark. The other three states with the triquark spin $S_{t}=3 / 2$ remain unmixed due to this interaction but can mix through the spin-spin interactions between (anti)quarks in the two separated shells - the heavy triquark and light diquark. As mentioned earlier, such types of spin-spin interactions are suppressed and neglected in this analysis. For the later three states, enumerated according to their entries in table 3 , the masses $m_{5,6,7}^{S 1}$ are the averages of the effective Hamiltonian over the states considered (here, the superscript denotes the $S$-wave pentaquark with the "bad" light diquark, $\left.S_{l d}=1\right)$ :

$$
m_{5,6,7}^{S 1}=M_{0}+\frac{1}{2}\left(\mathcal{K}_{c q}\right)_{\overline{3}}+\frac{1}{2}\left(\mathcal{K}_{q^{\prime} q^{\prime \prime}}\right)_{\overline{3}}+\frac{1}{2}\left(\mathcal{K}_{\bar{c} q}+\mathcal{K}_{\bar{c} c}\right) .
$$


So, one can see that taking into account only the spin-spin interactions inside the light diquark and heavy triquark, these states are degenerated in mass.

The pairs of states with $J^{P}=1 / 2^{-}$and $J^{P}=3 / 2^{-}$, after sandwiching the effective Hamiltonian, yield identical $(2 \times 2)$ mass matrices:

$$
M_{J=1 / 2,3 / 2}^{S 1}=M_{0}-\frac{1}{2}\left(\mathcal{K}_{c q}\right)_{\overline{3}}+\frac{1}{2}\left(\mathcal{K}_{q^{\prime} q^{\prime \prime}}\right) \overline{3}-\left(\mathcal{K}_{c q}\right)_{\overline{3}}\left(\begin{array}{cc}
1 & 0 \\
0 & -1
\end{array}\right)+\frac{1}{2}\left(\mathcal{K}_{\bar{c} q}+\mathcal{K}_{\bar{c} c}\right)\left(\begin{array}{cc}
0 & \sqrt{3} \\
\sqrt{3} & -2
\end{array}\right) .
$$

Diagonalizing the matrix, the states, being twice degenerate, have the masses:

$$
\begin{aligned}
& m_{1,3}^{S 1}=M_{0}-\frac{1}{4}\left(\mathcal{K}_{\bar{c} q}+\mathcal{K}_{\bar{c} c}\right)\left[2+r_{h d}-r_{l d}+2 \sqrt{3+\left(1-r_{h d}\right)^{2}}\right], \\
& m_{2,4}^{S 1}=M_{0}-\frac{1}{4}\left(\mathcal{K}_{\bar{c} q}+\mathcal{K}_{\bar{c} c}\right)\left[2+r_{h d}-r_{l d}-2 \sqrt{3+\left(1-r_{h d}\right)^{2}}\right],
\end{aligned}
$$

where $r_{h d}$ and $r_{l d}$ are defined in eq. (4.13). The averaged masses of the states with masses in (4.18) and (4.19) have the following value:

$$
\begin{aligned}
& \bar{m}_{12}^{S 1}=\frac{1}{2}\left[m_{1}^{S 1}+m_{2}^{S 1}\right]=M_{0}-\frac{1}{4}\left(\mathcal{K}_{\bar{c} q}+\mathcal{K}_{\bar{c} c}\right)\left(2+r_{h d}-r_{l d}\right), \\
& \bar{m}_{34}^{S 1}=\frac{1}{2}\left[m_{3}^{S 1}+m_{4}^{S 1}\right]=M_{0}-\frac{1}{4}\left(\mathcal{K}_{\bar{c} q}+\mathcal{K}_{\bar{c} c}\right)\left(2+r_{h d}-r_{l d}\right),
\end{aligned}
$$

and the mass gap between these average values and the mass $\bar{m}_{12}^{S 0}(4.14)$ is completely determined by the strength of the spin-spin interaction in the light diquark:

$$
\Delta \bar{m}_{12}^{S 1, S 0}=\bar{m}_{12}^{S 1}-\bar{m}_{12}^{S 0}=\left(\mathcal{K}_{\bar{c} q}+\mathcal{K}_{\bar{c} c}\right) r_{l d}=2\left(\mathcal{K}_{q^{\prime} q^{\prime \prime}}\right) \overline{3} .
$$

The degeneracy is lifted if the spin-spin interactions between the triquark and the lightdiquark constituents are taken into account as suggested by Maiani et al. in the Model I [35].

\section{$4.2 \quad P$-wave pentaquarks}

As it was discussed earlier in section 3 , one needs to include the terms dependent on the internal angular momenta of the pentaquark system which can be written as additional terms (3.6) and (3.7) in the pentaquark effective Hamiltonian.

Let the vector states $\left|S_{h d}, S_{t}, L_{t} ; S_{l d}, L_{l d} ; S, L\right\rangle_{J}$, appearing in tables 4 and 5 , be denoted as $\left|L,\left(S_{t}, S_{l d}\right)_{S} ; J\right\rangle$ and transformed to the state $\left|\left(L, S_{t}\right)_{J_{t}}, S_{l d} ; J\right\rangle$. After the transformation (4.4) is applied, we get:

$$
\left|L,\left(S_{t}, S_{l d}\right)_{S} ; J\right\rangle=\sum_{J_{t}}(-1)^{S_{t}+S_{l d}+L+J} \sqrt{(2 S+1)\left(2 J_{t}+1\right)}\left\{\begin{array}{ccc}
L & S_{t} & J_{t} \\
S_{l d} & J & S
\end{array}\right\}\left|\left(L, S_{t}\right)_{J_{t}}, S_{l d} ; J\right\rangle .
$$

The required matrix elements can be written in the form:

$$
\begin{aligned}
& { }_{J}\left\langle S_{h d}, S_{t}, L_{t} ; S_{l d}, L_{l d} ; S^{\prime}, L\left|2 A_{t}\left(\mathbf{L} \cdot \mathbf{S}_{t}\right)\right| S_{h d}, S_{t}, L_{t} ; S_{l d}, L_{l d} ; S, L\right\rangle_{J} \\
& =A_{t}(-1)^{2 S_{t}+2 J} \sqrt{(2 S+1)\left(2 S^{\prime}+1\right)} \sum_{J_{t}}\left(2 J_{t}+1\right) \\
& \quad \times\left[J_{t}\left(J_{t}+1\right)-S_{t}\left(S_{t}+1\right)-2\right]\left\{\begin{array}{ccc}
1 & S_{t} & J_{t} \\
S_{l d} & J & S
\end{array}\right\}\left\{\begin{array}{ccc}
1 & S_{t} & J_{t} \\
S_{l d} & J & S^{\prime}
\end{array}\right\},
\end{aligned}
$$

where the value of the orbital angular momentum, $L=1$, is substituted. 
Next, we denote the state $\left|S_{h d}, S_{t}, L_{t} ; S_{l d}, L_{l d} ; S, L\right\rangle_{J}$ as $\left|L,\left(S_{l d}, S_{t}\right)_{S} ; J\right\rangle$ and transform it to the state $\left|\left(L, S_{l d}\right)_{J_{l d}}, S_{t} ; J\right\rangle$. After the transformation (4.4) is applied, we get:

$$
\left|L,\left(S_{l d}, S_{t}\right)_{S} ; J\right\rangle=\sum_{J_{l d}}(-1)^{S_{t}+S_{l d}+L+J} \sqrt{(2 S+1)\left(2 J_{l d}+1\right)}\left\{\begin{array}{ccc}
L & S_{l d} & J_{l d} \\
S_{t} & J & S
\end{array}\right\}\left|\left(L, S_{l d}\right)_{J_{l d}}, S_{t} ; J\right\rangle .
$$

With this, the matrix elements can be written as:

$$
\begin{aligned}
& { }_{J}\left\langle S_{h d}, S_{t}, L_{t} ; S_{l d}, L_{l d} ; S^{\prime}, L\left|2 A_{l d}\left(\mathbf{L} \cdot \mathbf{S}_{l d}\right)\right| S_{h d}, S_{t}, L_{t} ; S_{l d}, L_{l d} ; S, L\right\rangle_{J} \\
& =A_{l d}(-1)^{2 S_{t}+2 J} \sqrt{(2 S+1)\left(2 S^{\prime}+1\right)} \sum_{J_{l d}}\left(2 J_{l d}+1\right) \\
& \quad \times\left[J_{l d}\left(J_{l d}+1\right)-S_{l d}\left(S_{l d}+1\right)-2\right]\left\{\begin{array}{ccc}
1 & S_{l d} & J_{l d} \\
S_{t} & J & S
\end{array}\right\}\left\{\begin{array}{ccc}
1 & S_{l d} & J_{l d} \\
S_{t} & J & S^{\prime}
\end{array}\right\},
\end{aligned}
$$

where again the value of the orbital angular momentum, $L=1$, is substituted.

We calculate the mass spectrum of the $P$-states, starting with the pentaquark states with the "good" light diquark from table 4 . From the seven states presented there, two states with $J^{P}=1 / 2^{+}$and the other two with $J^{P}=3 / 2^{+}$, both pairs having the triquark spin $S_{t}=1 / 2$, mix due to the spin-spin interaction of the charm antiquark and heavy diquark. The other three states with the triquark spin $S_{t}=3 / 2$ remain unmixed due to this interaction but can mix through the spin-spin interactions between the (anti)quarks entering two separated shells - the heavy triquark and light diquark, as also discussed earlier for the $S$-wave states. As mentioned earlier, such types of spin-spin interactions are suppressed and neglected in this analysis. From the terms entering the angular-momentum dependent parts (3.6) and (3.7) of the effective Hamiltonian, one obtains the spin-orbit, orbital, and tensor contributions to matrix elements. For the three states, enumerated as the fifth, sixth and seventh according to their rows in table 4 , the masses $m_{5,6,7}^{P 0}$ are the matrix elements of the effective Hamiltonian over these states (here, the superscript denotes the $P$-wave pentaquark with the "good" light diquark, $\left.S_{l d}=0\right)$ :

$$
\begin{aligned}
& m_{5}^{P 0}=M_{0}+\frac{1}{2}\left(\mathcal{K}_{c q}\right)_{\overline{3}}-\frac{3}{2}\left(\mathcal{K}_{q^{\prime} q^{\prime \prime}}\right)_{\overline{3}}+\frac{1}{2}\left(\mathcal{K}_{\bar{c} q}+\mathcal{K}_{\bar{c} c}\right)+B-5 A_{t}, \\
& m_{6}^{P 0}=m_{5}^{P 0}+3 A_{t}, \quad m_{7}^{P 0}=m_{5}^{P 0}+8 A_{t} .
\end{aligned}
$$

These states are non-degenerate due to the triquark spin-orbit interaction, i.e., $A_{t} \neq 0$.

The pair of states with $J^{P}=1 / 2^{-}$, after sandwiching the effective Hamiltonian, yields the following $(2 \times 2)$ mass matrix:

$M_{J=1 / 2}^{P 0}=M_{0}-\frac{1}{2}\left(\mathcal{K}_{c q}\right)_{\overline{3}}-\frac{3}{2}\left(\mathcal{K}_{q^{\prime} q^{\prime \prime}}\right)_{\overline{3}}-\left(\mathcal{K}_{c q}\right)_{\overline{3}}\left(\begin{array}{rr}1 & 0 \\ 0 & -1\end{array}\right)+\frac{1}{2}\left(\mathcal{K}_{\bar{c} q}+\mathcal{K}_{\bar{c} c}\right)\left(\begin{array}{cc}0 & \sqrt{3} \\ \sqrt{3} & -2\end{array}\right)+B-2 A_{t}$,

while the masses of the pair with $J^{P}=3 / 2^{-}$are determined by the matrix:

$$
M_{J=3 / 2}^{P 0}=M_{0}-\frac{1}{2}\left(\mathcal{K}_{c q}\right)_{\overline{3}}-\frac{3}{2}\left(\mathcal{K}_{q^{\prime} q^{\prime \prime}}\right)_{\overline{3}}-\left(\mathcal{K}_{c q}\right)_{\overline{3}}\left(\begin{array}{rr}
1 & 0 \\
0 & -1
\end{array}\right)+\frac{1}{2}\left(\mathcal{K}_{\bar{c} q}+\mathcal{K}_{\bar{c} c}\right)\left(\begin{array}{cc}
0 & \sqrt{3} \\
\sqrt{3} & -2
\end{array}\right)+B+A_{t} .
$$


Diagonalizing these matrices, we get the masses:

$$
\begin{aligned}
& m_{1}^{P 0}=M_{0}-\frac{1}{4}\left(\mathcal{K}_{\bar{c} q}+\mathcal{K}_{\bar{c} c}\right)\left[2+r_{h d}+3 r_{l d}+2 \sqrt{3+\left(1-r_{h d}\right)^{2}}\right]+B-2 A_{t} \\
& m_{2}^{P 0}=M_{0}-\frac{1}{4}\left(\mathcal{K}_{\bar{c} q}+\mathcal{K}_{\bar{c} c}\right)\left[2+r_{h d}+3 r_{l d}-2 \sqrt{3+\left(1-r_{h d}\right)^{2}}\right]+B-2 A_{t} \\
& m_{3,4}^{P 0}=m_{1,2}^{P 0}+3 A_{t}
\end{aligned}
$$

where $r_{h d}$ and $r_{l d}$ are defined in eq. (4.13).

We continue the calculations of the mass spectrum for the pentaquark states with the "bad" light diquark from table 5. From the eighteen states presented, there are six states with $J^{P}=1 / 2^{+}$, seven with $J^{P}=3 / 2^{+}$, four with $J^{P}=5 / 2^{+}$, and the last one with $J^{P}=7 / 2^{+}$. Except for the $J^{P}=7 / 2^{+}$state, all the others mix due to the spinspin interactions, but also because of the spin-orbit and tensor terms (3.6) and (3.7) in the effective Hamiltonian. For the last state, enumerated as the eighteenth according to table 5 , the mass $m_{18}^{P 1}$ is the average of the effective Hamiltonian over this state (here, the superscript denotes the $P$-wave pentaquark with the "bad" light diquark, $S_{l d}=1$ ):

$$
m_{18}^{P 1}=M_{0}+\frac{1}{2}\left(\mathcal{K}_{c q}\right)_{\overline{3}}+\frac{1}{2}\left(\mathcal{K}_{q^{\prime} q^{\prime \prime}}\right)_{\overline{3}}+\frac{1}{2}\left(\mathcal{K}_{\bar{c} q}+\mathcal{K}_{\bar{c} c}\right)+B+3 A_{t}+2 A_{l d}-\frac{3}{5} b
$$

As mentioned earlier, there are four states with spin-parity $J^{P}=5 / 2^{+}$which can be divided into two pairs according to their mixing mechanism: the fifth and the tenth states in table 5 mix due to the spin-spin interaction while for the fifteenth and the seventeenth states, their mixing is determined by the spin-orbit and tensor interactions. We start from the first of the mentioned two states. Their mass matrix is as follows:

$$
\begin{aligned}
M_{J=5 / 2}^{P 1}= & M_{0}-\frac{1}{2}\left(\mathcal{K}_{c q}\right)_{\overline{3}}+\frac{1}{2}\left(\mathcal{K}_{q^{\prime} q^{\prime \prime}}\right)_{\overline{3}}-\left(\mathcal{K}_{c q}\right)_{\overline{3}}\left(\begin{array}{rr}
1 & 0 \\
0 & -1
\end{array}\right)+\frac{1}{2}\left(\mathcal{K}_{\bar{c} q}+\mathcal{K}_{\bar{c} c}\right)\left(\begin{array}{rr}
0 & \sqrt{3} \\
\sqrt{3} & -2
\end{array}\right) \\
& +B+A_{t}+2 A_{l d}-\frac{1}{5} b .
\end{aligned}
$$

Diagonalizing the matrix, we obtain the masses:

$$
\begin{aligned}
& m_{14}^{P 1}=M_{0}-\frac{1}{4}\left(\mathcal{K}_{\bar{c} q}+\mathcal{K}_{\bar{c} c}\right)\left[2+r_{h d}-r_{l d}+2 \sqrt{3+\left(1-r_{h d}\right)^{2}}\right]+B+A_{t}+2 A_{l d}-\frac{1}{5} b \\
& m_{15}^{P 1}=M_{0}-\frac{1}{4}\left(\mathcal{K}_{\bar{c} q}+\mathcal{K}_{\bar{c} c}\right)\left[2+r_{h d}-r_{l d}-2 \sqrt{3+\left(1-r_{h d}\right)^{2}}\right]+B+A_{t}+2 A_{l d}-\frac{1}{5} b
\end{aligned}
$$

For the remaining two states, the mass matrix has the form:

$$
\begin{aligned}
\tilde{M}_{J=5 / 2}^{P 1}= & M_{0}+\frac{1}{2}\left(\mathcal{K}_{c q}\right)_{\overline{3}}+\frac{1}{2}\left(\mathcal{K}_{q^{\prime} q^{\prime \prime}}\right)_{\overline{3}}+\frac{1}{2}\left(\mathcal{K}_{\bar{c} q}+\mathcal{K}_{\bar{c} c}\right)+B \\
& +\frac{1}{5} A_{t}\left(\begin{array}{cc}
11 & 2 \sqrt{21} \\
2 \sqrt{21} & -6
\end{array}\right)+\frac{2}{5} A_{l d}\left(\begin{array}{cc}
2 & \sqrt{21} \\
\sqrt{21} & -2
\end{array}\right)-\frac{1}{250} b\left(\begin{array}{cc}
584 & 15 \sqrt{21} \\
15 \sqrt{21} & 24
\end{array}\right) .
\end{aligned}
$$


So, the masses of the pentaquarks are two eigenvalues of this matrix:

$$
\begin{aligned}
m_{16}^{P 1}= & M_{0}+\frac{1}{4}\left(\mathcal{K}_{\bar{c} q}+\mathcal{K}_{\bar{c} c}\right)\left[2+r_{h d}+r_{l d}\right]+B+\frac{1}{2} A_{t}-\frac{152}{125} b \\
& -\frac{1}{50} \sqrt{\left(85 A_{t}+40 A_{l d}-56 b\right)^{2}+21\left(20 A_{t}+20 A_{l d}-3 b\right)^{2}}, \\
m_{17}^{P 1}= & M_{0}+\frac{1}{4}\left(\mathcal{K}_{\bar{c} q}+\mathcal{K}_{\bar{c} c}\right)\left[2+r_{h d}+r_{l d}\right]+B+\frac{1}{2} A_{t}-\frac{152}{125} b \\
& +\frac{1}{50} \sqrt{\left(85 A_{t}+40 A_{l d}-56 b\right)^{2}+21\left(20 A_{t}+20 A_{l d}-3 b\right)^{2}} .
\end{aligned}
$$

Further, there are seven states with the spin-parity $J^{P}=3 / 2^{+}$which can be separated according to the triquark spin $S_{t}=1 / 2$ (four states) and $S_{t}=3 / 2$ (three states) as shown in table 5 . The former four states mix under the spin-spin, spin-orbit and tensor interactions while three states with $S_{t}=3 / 2$ mix due to the spin-orbit and tensor interactions only. The details of the mass derivation can be found in appendix B; here we present explicit equations for the masses only:

$$
m_{11,12,13}^{P 1}=M_{0}+\frac{1}{4}\left(\mathcal{K}_{\bar{c} q}+\mathcal{K}_{\bar{c} c}\right)\left(2+r_{h d}+r_{l d}\right)+B-\frac{4}{3}\left(A_{t}+A_{l d}\right)-\frac{3622}{1125} b+\lambda_{3,2,1},
$$

where $\lambda_{1,2,3}$ are determined in eq. (B.10).

To derive the expressions for the masses of the four states with the spin-parity $J^{P}=$ $3 / 2^{+}$and the triquark spin $S_{t}=1 / 2$, one needs to find the eigenvalues of a non-diagonal symmetric $(4 \times 4)$ matrix. The explicit form of the mass matrix and the details of calculations can be found in appendix B. The set of masses is as follows:

$$
\begin{aligned}
& m_{7}^{P 1}=M_{0}+B-\frac{A_{t}}{2}-\frac{14}{15} b-\frac{1}{4}\left(\mathcal{K}_{\bar{c} q}+\mathcal{K}_{\bar{c} c}\right)\left(2+r_{h d}-r_{l d}+2 \sqrt{3+\left(1-r_{h d}\right)^{2}}\right)-\mu^{(3 / 2),} \\
& m_{8}^{P 1}=M_{0}+B-\frac{A_{t}}{2}-\frac{14}{15} b-\frac{1}{4}\left(\mathcal{K}_{\bar{c} q}+\mathcal{K}_{\bar{c} c}\right)\left(2+r_{h d}-r_{l d}+2 \sqrt{3+\left(1-r_{h d}\right)^{2}}\right)+\mu^{(3 / 2),} \\
& m_{9}^{P 1}=M_{0}+B-\frac{A_{t}}{2}-\frac{14}{15} b-\frac{1}{4}\left(\mathcal{K}_{\bar{c} q}+\mathcal{K}_{\bar{c} c}\right)\left(2+r_{h d}-r_{l d}-2 \sqrt{3+\left(1-r_{h d}\right)^{2}}\right)-\mu^{(3 / 2),} \\
& m_{10}^{P 1}=M_{0}+B-\frac{A_{t}}{2}-\frac{14}{15} b-\frac{1}{4}\left(\mathcal{K}_{\bar{c} q}+\mathcal{K}_{\bar{c} c}\right)\left(2+r_{h d}-r_{l d}-2 \sqrt{3+\left(1-r_{h d}\right)^{2}}\right)+\mu^{(3 / 2)},
\end{aligned}
$$

where

$$
\mu^{(3 / 2)}=\frac{1}{30} \sqrt{\left(5 A_{t}+40 A_{l d}-12 b\right)^{2}+5\left(20 A_{t}+20 A_{l d}+3 b\right)^{2}} .
$$

Finally, there are six states with the spin-parity $J^{P}=1 / 2^{+}$which can be divided according to the triquark spin $S_{t}=1 / 2$ (four states) and $S_{t}=3 / 2$ (two states) as shown in table 5 . The four states mix under the spin-spin, spin-orbit and tensor interactions while the two states with $S_{t}=3 / 2 \mathrm{mix}$ through the spin-orbit and tensor interactions only. The mass matrix of the later two states is as follows:

$$
\begin{aligned}
\tilde{M}_{J=1 / 2}^{P 1}= & M_{0}+\frac{1}{2}\left(\mathcal{K}_{c q}\right)_{\overline{3}}+\frac{1}{2}\left(\mathcal{K}_{q^{\prime} q^{\prime \prime}}\right)_{\overline{3}}+\frac{1}{2}\left(\mathcal{K}_{\bar{c} q}+\mathcal{K}_{\bar{c} c}\right)+B-\frac{4}{3} b \\
& -\frac{1}{3} A_{t}\left(\begin{array}{cc}
10 & -2 \sqrt{5} \\
-2 \sqrt{5} & 11
\end{array}\right)+\frac{2}{3} A_{l d}\left(\begin{array}{cc}
2 & \sqrt{5} \\
\sqrt{5} & -2
\end{array}\right)+\frac{7}{2 \sqrt{5}} b\left(\begin{array}{ll}
0 & 1 \\
1 & 0
\end{array}\right) .
\end{aligned}
$$


The pentaquark masses are the two eigenvalues of this matrix:

$$
\begin{aligned}
m_{5}^{P 1}= & M_{0}+\frac{1}{4}\left(\mathcal{K}_{\bar{c} q}+\mathcal{K}_{\bar{c} c}\right)\left[2+r_{h d}+r_{l d}\right]+B-\frac{7}{2} A_{t}-\frac{4}{3} b \\
& -\frac{1}{6 \sqrt{5}} \sqrt{5\left(A_{t}+8 A_{l d}\right)^{2}+\left(20 A_{t}+20 A_{l d}+21 b\right)^{2}}, \\
m_{6}^{P 1}= & M_{0}+\frac{1}{4}\left(\mathcal{K}_{\bar{c} q}+\mathcal{K}_{\bar{c} c}\right)\left[2+r_{h d}+r_{l d}\right]+B-\frac{7}{2} A_{t}-\frac{4}{3} b \\
& +\frac{1}{6 \sqrt{5}} \sqrt{5\left(A_{t}+8 A_{l d}\right)^{2}+\left(20 A_{t}+20 A_{l d}+21 b\right)^{2}} .
\end{aligned}
$$

For the last four states with the spin-parity $J^{P}=1 / 2^{+}$and triquark spin $S_{t}=1 / 2$, the mass matrix is again a non-diagonal symmetric $(4 \times 4)$ matrix. It is written explicitly in appendix B and details of calculations of its eigenvalues can be also found there. The resulting set of masses is presented below:

$$
\begin{aligned}
m_{1}^{P 1}= & M_{0}+B-\frac{A_{t}}{2}-3 A_{l d}-\frac{31}{30} b \\
& -\frac{1}{4}\left(\mathcal{K}_{\bar{c} q}+\mathcal{K}_{\bar{c} c}\right)\left(2+r_{h d}-r_{l d}+2 \sqrt{3+\left(1-r_{h d}\right)^{2}}\right)-\mu^{(1 / 2)}, \\
m_{2}^{P 1}= & M_{0}+B-\frac{A_{t}}{2}-3 A_{l d}-\frac{31}{30} b \\
& -\frac{1}{4}\left(\mathcal{K}_{\bar{c} q}+\mathcal{K}_{\bar{c} c}\right)\left(2+r_{h d}-r_{l d}+2 \sqrt{3+\left(1-r_{h d}\right)^{2}}\right)+\mu^{(1 / 2)}, \\
m_{3}^{P 1}= & M_{0}+B-\frac{A_{t}}{2}-3 A_{l d}-\frac{31}{30} b \\
& -\frac{1}{4}\left(\mathcal{K}_{\bar{c} q}+\mathcal{K}_{\bar{c} c}\right)\left(2+r_{h d}-r_{l d}-2 \sqrt{3+\left(1-r_{h d}\right)^{2}}\right)-\mu^{(1 / 2)}, \\
m_{4}^{P 1}= & M_{0}+B-\frac{A_{t}}{2}-3 A_{l d}-\frac{31}{30} b \\
& -\frac{1}{4}\left(\mathcal{K}_{\bar{c} q}+\mathcal{K}_{\bar{c} c}\right)\left(2+r_{h d}-r_{l d}-2 \sqrt{3+\left(1-r_{h d}\right)^{2}}\right)+\mu^{(1 / 2)},
\end{aligned}
$$

where

$$
\mu^{(1 / 2)}=\frac{1}{30} \sqrt{25\left(7 A_{t}+2 A_{l d}+3 b\right)^{2}+2\left(20 A_{t}+20 A_{l d}+21 b\right)^{2}} .
$$

With these expressions, the analytical calculations of the pentaquark mass spectrum in Model II is done and we present the numerical estimates of the pentaquark masses in the next section.

\section{Hidden-charm pentaquark mass predictions}

\section{$5.1 \quad$ Input parameters}

Working within the Constituent Quark-Diquark Model [56], input parameters are the masses of the constituents, charm quark and two diquarks, spin-spin couplings, and other parameters related with the orbital or radial excitations. In the present paper we analyse the ground-state pentaquarks and their first orbital excitations only. To estimate the constituent quark masses, there are two possibilities: either extract them from the masses of 


\begin{tabular}{|cccccc|}
\hline Diquark content & {$[u d]$} & {$[s q]$} & $\{s s\}$ & {$[c q]$} & {$[c s]$} \\
\hline Diquark mass $(\mathrm{MeV})$ & 576 & 800 & 1099 & 1976 & 2105 \\
\hline
\end{tabular}

Table 6. Constituent diquark masses (in $\mathrm{MeV}$ ) used for calculating the hidden-charm pentaquark mass spectrum. Here, $q$ denotes $u$ - and $d$-quarks and isospin symmetry is assumed. The brackets [...] and $\{\ldots\}$ indicate the antisymmetric $(S=0)$ and symmetric $(S=1)$ spin configurations of the quarks in the diquark, respectively. (Taken from [62] for light diquarks and from [15] for the heavy-light ones).

known mesons or from baryons [15]. Quark masses obtained from the baryon spectrum are larger by typically $50 \mathrm{MeV}$ [15]. This can be exemplified by the charm quark mass, which is estimated as $m_{c}^{m}=1667 \mathrm{MeV}$ from the $D$-meson spectrum, as opposed to $m_{c}^{b}=1710 \mathrm{MeV}$ from the charm baryon masses, yielding a mass difference of $43 \mathrm{MeV}$ [15]. In particular, with the $m_{c}^{b}$ value as an input, predictions for the charm baryon masses were obtained [61] which differ from the experimentally observed masses [3] by about $10 \mathrm{MeV}$, which may be viewed as an error on the input charm-quark mass. We use $m_{c}^{b}$ in the numerical analysis. Based on the same arguments, we accept $m_{q}^{b}=(362 \pm 10) \mathrm{MeV}$ and $m_{s}^{b}=(540 \pm 10) \mathrm{MeV}$ as the light and strange quark masses, respectively [15].

The diquark masses are presented in table 6 . In contrast to quarks, which have spin $S_{q}=1 / 2$, diquarks, being composite objects, have two possible spin configurations from which the antisymmetric one corresponding to the diquark spin $S=0$ is energetically more favorable. Both configurations are allowed if the flavors of the quarks are different, indicated by right brackets, [...], in table 6 (for $S=0$ ), and by curly brackets, $\{\ldots\}$, (for $S=1$ ). For the diquarks, having two quarks of the same flavor, only the spin-symmetric configuration is allowed by Bose statistics, as indicated for the case of the diquark with two $s$-quarks in table 6 .

In the limit of the exact isospin symmetry, the light $u$ - and $d$-quarks are massdegenerate and denoted by $q$ in table 6 . For the lightest diquark, $[u d]$, and its $\mathrm{SU}(3)_{F^{-}}$ symmetry partners, $[s u]$ and $[s d]$, the masses are taken from [62] as well as the mass of the double strange diquark, $\{s s\}$. The masses of the charm diquarks, $[c q]$ and $[c s]$, are borrowed from [15]. To get the diquark-mass error estimates, one should compare baryon masses predicted within this model and in experiment. Based on the analysis presented in [61], in which the predicted and measured mass differences for unflavored (i.e., having $u$ - or $d$-quarks), strange and charm baryons do not exceed $15 \mathrm{MeV}$, we take it as a measure of the uncertainty in diquark masses.

The spin-spin couplings, $\mathcal{K}_{\bar{Q} Q^{\prime}}$ and $\left(\mathcal{K}_{Q Q^{\prime}}\right) \overline{3}$, extracted from the spectra of mesons and baryons, respectively, are presented in table 7 . These values are taken from [15] except for $\left(\mathcal{K}_{c c}\right) \overline{3}$, which is from [61]. The factor two difference in $\mathcal{K}_{\bar{c} c}$ and $\left(\mathcal{K}_{c c}\right)_{\overline{3}}$ reflects the fact that the one-gluon exchange assumption is used to relate these couplings. Concerning the $\left(\mathcal{K}_{c q}\right)_{\overline{3}}$-coupling, it follows from table 7 that the value $\left(\mathcal{K}_{c q}\right)_{\overline{3}}=15 \mathrm{MeV}$ extracted from the charm baryon spectroscopy is numerically substantially smaller than all others. In fact, this is the spin-spin interaction between the charm quark and the two quarks from a light diquark, but not between the quarks within the charm diquark $[c q]$, which is 


\begin{tabular}{|ccccccc|}
\hline Couplings $(\mathrm{MeV})$ & $q q^{\prime}$ & $s q$ & $s s$ & $c q$ & $c s$ & $c c$ \\
\hline $\mathcal{K}_{\bar{Q} Q^{\prime}}$ & 318 & 200 & 103 & 70 & 72 & 113 \\
$\left(\mathcal{K}_{Q Q^{\prime}}\right)_{\overline{3}}$ & 98 & 59 & 23 & 15 & 50 & 57 \\
\hline
\end{tabular}

Table 7. Spin-spin couplings, $\mathcal{K}_{\bar{Q} Q^{\prime}}$ and $\left(\mathcal{K}_{Q Q^{\prime}}\right)_{\overline{3}}$, extracted from the spectra of mesons and baryons, respectively (borrowed from [15] and for $c c$ from [61]). Here, $q^{(\prime)}$ denotes $u$ - and $d$-quarks and isospin symmetry is assumed.

estimated as $\left(\mathcal{K}_{c q}\right)_{\overline{3}}=67 \mathrm{MeV}[15,56]$. The later value was obtained from the Constituent Quark-Diquark Model analysis of the hidden-charm exotic mesons in which the underlying structure is assumed to be a charm diquark and charm antidiquark. In our numerical estimates, we use the later value. The spin-spin coupling obtained from the charm baryons can be used to determine $\left(\tilde{\mathcal{K}}_{c q}\right)_{\overline{3}}$ in eq. (3.4), associated with the spin-spin interactions between the charm quark in the doubly-heavy triquark and quarks from the light diquark. The same is also true for the spin-spin couplings between the charm and strange quarks, for which the value $\left(\mathcal{K}_{c s}\right)_{\overline{3}}=2 \mathrm{MeV}[57,59]^{1}$ follows from the analysis of the narrow orbitallyexcited $\Omega_{c}^{*}$-baryons recently observed by the LHCb [63] and Belle [64] collaborations. To understand the uncertainty of $\left(\mathcal{K}_{c s}\right) \overline{3}$, we performed the $\chi^{2}$-analysis based on the masses of these baryons $[63,64]$, which yields $\left(\mathcal{K}_{c s}\right)_{\overline{3}}=(2.01 \pm 0.20) \mathrm{MeV}$. The details of this analysis are in appendix $\mathrm{C}$. This yields $10 \%$ error on $\left(\mathcal{K}_{c s}\right)_{\overline{3}}$, which we assume as an uncertainty on all the spin-spin couplings. The pentaquark masses involve the ratios of the couplings (4.13) which for the input values are evaluated as: $r_{h d}=0.73$ and $r_{l d}=1.07$. According to the Model II, these ratios should be of order one which explicitly demonstrates that the strengths of all possible spin-spin interactions inside the diquarks and triquark are approximately the same.

In estimating the $P$-wave pentaquark mass spectrum, values of the couplings in the orbital angular momentum term and the spin-orbit ones are required. In the previous analysis $[51,52]$, the spin-orbit coupling was taken to be $A_{\mathcal{P}}=52 \mathrm{MeV}$, which follows from the analysis of the vector hidden-charm $Y$-tetraquarks. Updated analysis of these states in [57], which takes into account in addition the tensor interaction between the diquark and antidiquark, results in the smaller value, $a_{Y}=(22 \pm 3) \mathrm{MeV}$. A similar analysis $[57,59]$, performed for the orbitally-excited $\Omega_{c}$-baryons, yields even smaller values, ${ }^{2}$ $a_{1}=(13.45 \pm 0.13) \mathrm{MeV}$ and $a_{2}=(12.94 \pm 0.36) \mathrm{MeV}$, for the spin-orbit interactions of the $\{s s\}$-diquark and charm antiquark, respectively. The uncertainties in these values result from the $\chi^{2}$-analysis of the $\Omega_{c}^{*}$-baryon masses $[63,64]$ and the details of this analysis are also presented in appendix $\mathrm{C}$. The errors in these couplings are typically a few percent and are substantially smaller than the relative error from the $Y$-tetraquark spectrum which is approximately $14 \%$.

\footnotetext{
${ }^{1}$ This value differs by a factor of two from the original estimate presented in $[57,59]$ due to the coupling redefinition, i.e., $\left(\mathcal{K}_{c s}\right)_{\overline{3}}=c / 2$.

${ }^{2}$ The original values $\left(a_{1}=26.95 \mathrm{MeV}\right.$ and $\left.a_{2}=25.75 \mathrm{MeV}\right)$ are twice the ones in the text because of different definitions in the effective Hamiltonian.
} 
For $P$-wave pentaquarks, the spin-orbit couplings are responsible for the interactions of the triquark and the light diquark, denoted by $A_{t}$ and $A_{l d}$, respectively, which are, in general, different. However, based on the observation that the couplings of the spin-orbit interaction in $\Omega_{c}^{*}$-baryons are close to each other, $a_{1} \simeq a_{2} \simeq 13 \mathrm{MeV}$, one can use the same approximate relation $A_{t} \simeq A_{l d}=13 \mathrm{MeV}$ in the pentaquark system but, as we shall show later, this is not supported by the data on the newly observed pentaquark states [1] for the plausible spin-parity assignment, as discussed in [34].

For the mass spectrum of orbitally-excited pentaquarks with the "bad" light diquark, the tensor coupling $b$ is required. It was extracted from the spectrum of the orbitallyexcited $\Omega_{c}$-baryons and $Y$-tetraquarks, yielding $b_{\Omega_{c}}=13.5 \mathrm{MeV}[57,59]^{3}$ and $b_{Y}=(-136 \pm$ 6) $\mathrm{MeV}$ [57], respectively. Thus, this coupling is quite different in the charm baryons and in the hidden-charm tetraquarks and, moreover, the central values are of opposite signs in these hadrons. Here, more experimental input is needed. To be definite, we use $b=(13.3 \pm 0.5) \mathrm{MeV}$, which is obtained from the orbitally-excited $\Omega_{c}$-baryons rather than from the $Y$-tetraquarks.

The last parameter to be specified is the orbital coupling $B$, several estimates of which are available from different hadrons. Some representative values are: $B(\bar{c} c)=457 \mathrm{MeV}$ [57], obtained from charmonia, $B\left(\Omega_{c}\right)=325 \mathrm{MeV}$, from $\Omega_{c}$-baryons, $B(Y)=362 \mathrm{MeV}$ and $B(Y)=505 \mathrm{MeV}$, from the hidden-charm $Y$-tetraquarks. ${ }^{4}$ In the analysis [51, 52], the coupling $B$ was derived from the experimentally observed mass of the $P_{c}(4450)^{+}$having the (preferred) spin-parity assignment $J^{P}=5 / 2^{+}$. The orbital coupling was determined from the $P_{c}(4450)^{+}$mass to be $B\left(P_{c}\right)=220 \mathrm{MeV}$ for the Model II [56]. With the observation of three narrow pentaquarks in the updated $\mathrm{LHCb}$ data, the experimental picture is changed drastically. Following the interpretation of these peaks as suggested in [34], which assumes that two resonances are orbitally-excited states and the lowest mass peak is the $S$-wave state, it is possible to determine the orbital coupling $B$ phenomenologically, discussed in the next subsection.

\subsection{Predictions for the hidden-charm unflavored pentaquarks}

To get an estimate of the mass spectrum of the ground-state hidden-charm unflavored pentaquarks, it is enough to know the diquark and charm quark masses and spin-spin couplings, discussed in detail in the previous subsection. For the $P$-wave pentaquark masses, the values of the orbital angular momentum coupling $B$, the spin-orbit couplings, $A_{t}$ and $A_{l d}$, and the tensor coupling $b$ are required. Some of these couplings can be determined from the measured masses of the observed resonances [1]. In this subsection we consider two possible assignments, both assume that the heavier states, $P_{c}(4440)^{+}$and $P_{c}(4457)^{+}$, are the $P$-wave pentaquarks, while the lowest mass $P_{c}(4312)^{+}$can be either $S$ - or $P$-wave state, and show that the second scenario is physically unacceptable. Assuming that the

\footnotetext{
${ }^{3}$ The $\chi^{2}$-analysis of the $\Omega_{c}^{*}$-baryons presented in appendix $C$ gives slightly smaller value $b_{\Omega_{c}}=(13.30 \pm$ $0.48) \mathrm{MeV}$.

${ }^{4}$ The values presented are determined by two possible assignments of the vector tetraquarks discussed in $[57]$.
} 
$P_{c}(4312)^{+}$pentaquark is an $S$-wave state, we predict the masses and $J^{P}$ quantum numbers of yet unmeasured resonances.

We discuss first the pentaquarks $P_{c}(4440)^{+}$and $P_{c}(4457)^{+}$. As they replace the former narrow $P_{c}(4450)^{+}$state with the preferred spin-parity $J^{P}=5 / 2^{+}$, we tentatively assign this spin-parity to one of the observed two states, $P_{c}(4457)^{+}$. In this case, the lighter partner, $P_{c}(4440)^{+}$, most probably has the spin-parity $J^{P}=3 / 2^{+}$. In the Model II by Maiani et al. [56], the mass splitting of the two positive-parity states is related to the spin-orbit coupling $A_{t}$ of the triquark:

$$
M\left[P_{c}(4457)^{+}\right]-M\left[P_{c}(4440)^{+}\right]=\left(17_{-4.5}^{+6.4}\right) \mathrm{MeV}=5 A_{t},
$$

where the error is obtained by adding the experimental errors on the masses in quadrature. From (5.1), the value of the coefficient $A_{t}$ follows immediately:

$$
A_{t}=\left(3.4_{-0.9}^{+1.3}\right) \mathrm{MeV} .
$$

It is not surprising that $A_{t}$ is found numerically small, as the doubly-heavy triquark is almost static.

The third narrow state $P_{c}(4312)^{+}$can have several $J^{P}$ assignments. Identifying it with the $J^{P}=3 / 2^{-}$state, one can work out the mass difference between this state and the heavier pentaquarks, $P_{c}(4440)^{+}$and $P_{c}(4457)^{+}$. This is determined by the orbital $B$ and the triquark spin-orbit $A_{t}$ couplings. The strength of the latter is already known from the $P_{c}(4440)^{+}$and $P_{c}(4457)^{+}$mass splitting (5.2), and the mass difference, say, between $P_{c}(4312)^{+}$and $P_{c}(4457)^{+}$, allows us to read off the strength of the orbital interaction:

$$
M\left[P_{c}(4457)^{+}\right]-M\left[P_{c}(4312)^{+}\right]=\left(145.4_{-7.1}^{+4.2}\right) \mathrm{MeV}=B+3 A_{t} .
$$

With $A_{t}$ from (5.2), we get $B=135 \mathrm{MeV}$. This is too small in comparison with the strengths of the orbital excitations in other hadrons [57], and, in particular, $B\left(\Omega_{c}\right)=$ $325 \mathrm{MeV}$, obtained from the $\Omega_{c}^{*}$-baryons. Moreover, the theoretically predicted masses of the $P_{c}(4440)^{+}$and $P_{c}(4457)^{+}$states with the value of $B \simeq 135 \mathrm{MeV}$ are found to be $\sim 70 \mathrm{MeV}$ below the experimental values. An error on $B$ can be obtained by performing the $\chi^{2}$-analysis of the experimental data. Assuming that the parameters $M_{0}, B$, and $A_{t}$ are free variables and all the spin-spin couplings are fixed, we obtain:

$$
M_{0}=(4333.9 \pm 3.9) \mathrm{MeV}, \quad B=(135.2 \pm 4.6) \mathrm{MeV}, \quad A_{t}=(3.4 \pm 1.1) \mathrm{MeV} .
$$

The best-fit value of $M_{0}$ comes out about $72 \mathrm{MeV}$ higher than the sum of the diquarks' and charm quark masses, $M_{0}=4262 \mathrm{MeV}$.

Alternatively, assuming that the spin-spin couplings and the constituent (quark and diquarks) masses are known, the strength of $B$ can also be determined from the masses of $P_{c}(4440)^{+}$and $P_{c}(4457)^{+}$only, as follows:

$$
B=\frac{1}{5}\left\{3 M\left[P_{c}(4440)^{+}\right]+2 M\left[P_{c}(4457)^{+}\right]\right\}-M_{0}-\frac{1}{4}\left(\mathcal{K}_{\bar{c} q}+\mathcal{K}_{\bar{c} c}\right)\left(2+r_{h d}-3 r_{l d}\right) .
$$

With the values of the other parameters already assigned, $B=207 \mathrm{MeV}$ reproduces the masses of the observed $P_{c}(4440)^{+}$and $P_{c}(4457)^{+}$states, shown in table 8 as the last two 
entries situated in the part labeled by $S_{l d}=0, L=1$ and inserted into the solid boxes. This value of $B$ is closer to the estimates in the hidden and open charm hadrons [57]. With these parameters, the mass of the third pentaquark $M=4240 \mathrm{MeV}$ with $J^{P}=3 / 2^{-}$, also shown in the solid box, is somewhat lower than the mass of the observed $P_{c}(4312)^{+}$peak, but is still in the right ball-park.

The second possibility is to assign the lowest mass state $P_{c}(4312)^{+}$with the one having the spin-parity $J^{P}=3 / 2^{+}$and mass $M=4361 \mathrm{MeV}$, or with $J^{P}=1 / 2^{+}$and $M=$ $4351 \mathrm{MeV}$. Both predictions are rather close to the observed pentaquark mass. In the case of $J^{P}=1 / 2^{+}$assignment for $P_{c}(4312)^{+}$, Model II predicts for the mass difference:

$$
\begin{aligned}
M\left[P_{c}(4440)^{+}\right]-M\left[P_{c}(4312)^{+}\right] & =\left(128.4_{-8.4}^{+4.4}\right) \mathrm{MeV} \\
& =\frac{1}{2}\left(\mathcal{K}_{\bar{c} q}+\mathcal{K}_{\bar{c} c}\right)\left(2+r_{h d}-\sqrt{3+\left(1-r_{h d}\right)^{2}}\right),
\end{aligned}
$$

which does not depend on $B$ and $A_{t}$ but is determined by the spin-spin couplings from the doubly-heavy triquark. If we fix the spin-spin coupling $\left(\mathcal{K}_{c q}\right)_{\overline{3}}=67 \mathrm{MeV}$, eq. (5.6) can be solved analytically with the roots:

$$
r_{h d}^{(1)}=0, \quad r_{h d}^{(2)}=\frac{2(3-2 \rho)}{\rho(2-\rho)},
$$

where $\rho=\left\{M\left[P_{c}(4440)^{+}\right]-M\left[P_{c}(4312)^{+}\right]\right\} /\left(\mathcal{K}_{c q}\right)_{\overline{3}}$. The first solution is unacceptable, as it implies $\mathcal{K}_{\bar{c} q}+\mathcal{K}_{\bar{c} c} \rightarrow \infty$. The second solution in (5.7) yields a negative and, hence, unphysical value for $r_{h d}$, if the ratio $\rho$ lies in the interval: $3 / 2<\rho<2$. With the pentaquark mass difference in $(5.6)$ and $\left(\mathcal{K}_{c q}\right)_{\overline{3}}=67 \mathrm{MeV}$, one obtains the value $\rho \simeq 1.92$, which is in the unphysical interval (the corresponding value of the spin-spin coupling ratio $\left.r_{h d}^{(2)} \simeq-10.4\right)$. The situation does not improve if we assign $J^{P}=3 / 2^{+}$to the third state. In this case, it has a higher mass than the $J^{P}=1 / 2^{+}$pentaquark by $3 A_{t} \simeq 10 \mathrm{MeV}$, thereby the r.h.s. of (5.6) gets an additional factor $-3 A_{t}$. There are again two solutions as in (5.7) but in $r_{h d}^{(2)}$ one should make the following replacement: $\rho \rightarrow \tilde{\rho}=\left\{M\left[P_{c}(4440)^{+}\right]-\right.$ $\left.M\left[P_{c}(4312)^{+}\right]+3 A_{t}\right\} /\left(\mathcal{K}_{c q}\right)_{\overline{3}}$. Numerically $\tilde{\rho} \simeq 2.07$, and with this value $r_{h d}^{(2)}$ becomes positive but too large, $r_{h d}^{(2)} \simeq 16$, which is again unacceptable. So, the assignment of the third state, $P_{c}(4312)^{+}$, with the orbitally-excited pentaquark is not tenable in the Model II.

It should be noted that the assignment of all the three observed states with the $P$-wave pentaquarks was also discussed in ref. [65]. In contrast to the one-gluon-exchange model, from which the effective Hamiltonian used in our paper follows, the effective Hamiltonian in [65] is based on a confining potential and an attractive flavor-spin interaction, resulting from the underlying chiral dynamics. Under the assumption that the flavor-spin interaction is dominant, the three $\mathrm{LHCb}$ states are then identified with the lowest-mass orbitallyexcited states. Moreover, in this model, the lowest $S$-wave state lies above the $P$-wave states. This obviously contradicts the predictions of our model discussed above.

We remark that the assignments we have discussed satisfy the heavy-quark symmetry, which means that the spin $S_{l d}=0$ of the light diquark in $\Lambda_{b}$-baryon is conserved in the decay to the charged pentaquark and $K^{-}$-meson. That is why, only pentaquarks with the "good" light diquark are considered. As the heavy-quark symmetry is not exact, pentaquarks with 


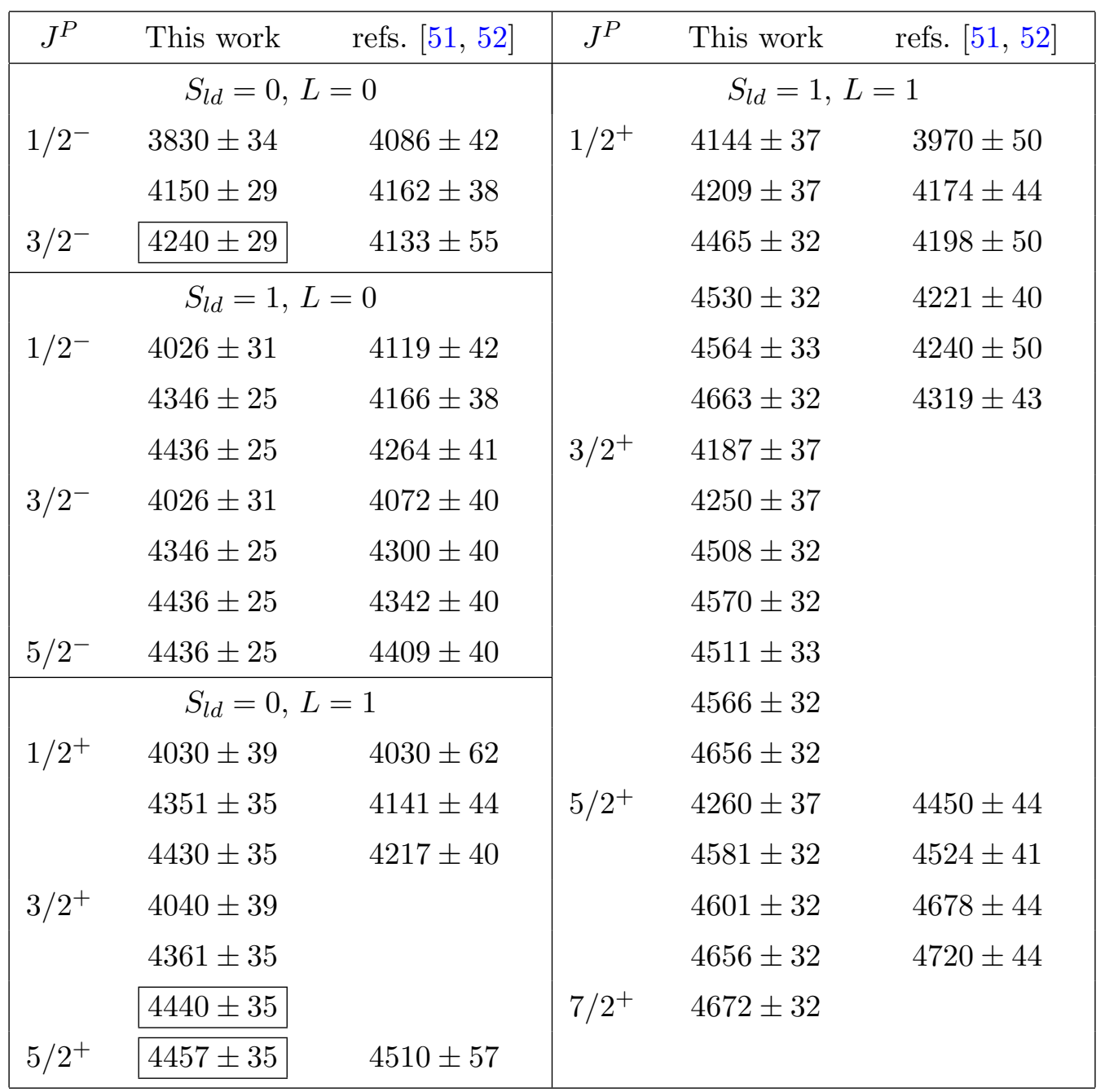

Table 8. Masses of the hidden-charm unflavored pentaquarks (in $\mathrm{MeV}$ ) and their comparison with the results presented in $[51,52]$. For the $P$-wave pentaquarks, the values of the orbital, spin-orbit and tensor couplings are taken from table 9 .

$S_{l d}=1$ can also be produced in the $\Lambda_{b}$-baryon decays, but being suppressed by $1 / m_{b}$, they are rather unlikely.

Masses of the hidden-charm unflavored pentaquarks are presented in table 8 , and compared with the results obtained in $[51,52]$. For the $P$-wave pentaquarks, the values of the orbital, spin-orbit and tensor couplings are taken from table 9. The threshold for the observed pentaquarks in the $P_{c}^{+} \rightarrow J / \psi+p$ decay mode is $M_{J / \psi p}^{\mathrm{thr}}=m_{J / \psi}+m_{p}=$ 4035.17 MeV [3]. With the masses given in table 8, there are two states with the "good" light diquark $\left(S_{l d}=0\right)$, the $J^{P}=1 / 2^{-}$state with the mass $M=3830 \mathrm{MeV}$ and the $J^{P}=1 / 2^{+}$state with the mass $M=4030 \mathrm{MeV}$, which lie below the $M_{J / \psi p}^{\mathrm{thr}}$ threshold. Also, the third state having $J^{P}=3 / 2^{+}$, with the mass $M=4040 \mathrm{MeV}$, may also lie below $M_{J / \psi p}^{\mathrm{thr}}$. There are also two states, $J^{P}=1 / 2^{-}$and $J^{P}=3 / 2^{-}$, with the "bad" light diquark $\left(S_{l d}=1\right)$, having the degenerate masses $M=4026 \mathrm{MeV}$, which also lie below the 


\begin{tabular}{|cccc|}
\hline$B$ & $A_{t}$ & $A_{l d}$ & $b$ \\
\hline $207 \pm 20$ & $3.4 \pm 1.1$ & $13.5 \pm 0.4$ & $13.3 \pm 0.5$ \\
\hline
\end{tabular}

Table 9. Input values (in $\mathrm{MeV}$ ) for the couplings required for the mass estimations of the $P$-wave pentaquarks. For the coupling $B$, the error is taken from (C.13).

$M_{J / \psi p}^{\mathrm{thr}}$ threshold. One of these states (with the mass $M=3830 \mathrm{MeV}$ ) is even below the threshold for the decay $P_{c}^{+} \rightarrow \eta_{c}+p$ with $M_{\eta_{c} p}^{\text {thr }}=m_{\eta_{c}}+m_{p}=3922 \mathrm{MeV}$ [3]. They will decay through the annihilation of the $c \bar{c}$-pair into light hadrons or a lepton pair, and hence will be narrower the $P_{c}^{+}$-resonances observed. It is conceivable that a dedicated search of narrow states in the $\mathrm{LHCb}$ data with improved statistics may reveal the existence of these pentaquark states. It is interesting to note that the virtual $J / \psi$-meson decay will also lead to an off-shell dilepton pair. Hence, a search for a resonance in the $p \mu^{+} \mu^{-}$(as well as $p e^{+} e^{-}$) may result into observing narrow structures. In addition, the other states shown in table 8 are also reachable in the $\Lambda_{b} \rightarrow J / \psi p K^{-}$decay, in particular, our model predicts a peak with the mass $M=(4150 \pm 29) \mathrm{MeV}$ below the lowest-mass observed pentaquark, $P_{c}(4312)^{+}$, and the other peak with a mass near $4350 \mathrm{MeV}$, being a combination of two $J^{P}=1 / 2^{+}$and $J^{P}=3 / 2^{+}$pentaquarks with close masses. The measured spectrum by the LHCb collaboration [1] has indications for such states, though more data are needed for a definite conclusion.

\subsection{Comparisons with the dynamical diquark model}

Before presenting the strange pentaquark spectrum, we compare some of our estimates in the non-strange sector with those obtained in the dynamical diquark model [54].

The common feature of our model and the dynamical diquark model is the assumption of point-like diquarks in the color-antitriplet representation, with the pentaquarks built from a diquark and a color-triplet triquark. However, the two approaches differ in their dynamical details and in assigning the flavors of the quarks in the diquarks and triquarks. In our model, we assume that the light diquark $\left[q^{\prime} q^{\prime \prime}\right]_{\overline{3}}$ is bound to the doubly-heavy triquark $\left[\bar{c}_{\overline{3}}[c q]_{\overline{3}}\right]_{3}$ and the orbital excitations in the pentaquark are due to the light-diquark orbital excitations (the doubly-heavy triquark is assumed to be in the $S$-wave). In the dynamical diquark model [54], both the diquark $[c q]_{\overline{3}}$ and the triquark $\left[\bar{c}_{\overline{3}}\left[q^{\prime} q^{\prime \prime}\right]_{\overline{3}}\right]_{3}$ are heavy objects, and orbital excitations are between them [54]. More importantly, in our analysis we take into account all sizable spin-spin, spin-orbit, orbital and tensor interactions between the charm antiquark and the diquarks, except for the spin-spin interaction between the constituents of the doubly-heavy triquark and the light diquark, which are assumed to be strongly suppressed. All these interactions are omitted in the current formulation of the dynamical diquark model and left for further study [54]. So, a lot of pentaquark states which differ in their masses in our model (see, for example, tables 2 and 4) are mass degenerate in the dynamical diquark model, which makes a numerical comparison rather difficult.

We also differ in the $J^{P}$ assignments of the states. In the dynamical diquark model [54], two possible assignments are suggested: the first one identifies the two pentaquark states 
with larger masses, $P_{c}(4440)^{+}$and $P_{c}(4457)^{+}$, with the states from the first orbitallyexcited multiplet called $\Sigma_{g}^{+}(1 P)$ (see table 7 in [54]). With this assignment, there is no place in the mass spectrum for the third narrow state, $P_{c}(4312)^{+}$, observed recently by the LHCb collaboration. This is not surprising, as also remarked in [54], since the strength of the orbital excitation in this assignment is about $\sim 400 \mathrm{MeV}$ for the states with the principle quantum number $n=1$, in accord with the corresponding excitation energy in charmonia. The observed mass splitting $\sim 140 \mathrm{MeV}$ is, therefore, impossible to accommodate with these assignment. So, the second possibility discussed in [54] is to put the lowest-mass state, $P_{c}(4312)^{+}$, in the first orbitally-excited multiplet $\Sigma_{g}^{+}(1 P)$, and in this case the two other states $P_{c}(4440)^{+}$and $P_{c}(4457)^{+}$are the members of the radially excited multiplet $\Sigma_{g}^{+}(2 S)$. The suggested spin-parity of the $P_{c}(4380)^{+}$-state is $J^{P}=5 / 2^{+}$(this means that the bottom of the $\Sigma_{g}^{+}(1 P)$-multiplet is fixed to the mass of the $P_{c}(4312)^{+}$ pentaquark) while one of the $P_{c}(4440)^{+}$- and $P_{c}(4457)^{+}$-states has $J^{P}=3 / 2^{-}$. In our model, all three observed pentaquark states have the principle quantum number $n=1$ and have the indicated parities: $J^{P}=3 / 2^{-}$for $P_{c}(4312)^{+}$and $J^{P}=3 / 2^{+}$and $J^{P}=5 / 2^{+}$ for $P_{c}(4440)^{+}$and $P_{c}(4457)^{+}$, respectively. We are able to explain quantitatively the mass splitting in the higher mass pentaquarks by the spin-orbit interaction, and the difference between the lower- and higher-mass states, to a large extent, by the orbital interaction. The strength of the latter interaction was determined as $\sim 200 \mathrm{MeV}$, which is smaller by $\sim 100 \mathrm{MeV}$ compared to charmonia, but still acceptable as the size of the pentaquark and, hence, the distance from the doubly-heavy triquark to the light diquark, is anticipated to be significantly larger than the hadronic size of the charmonium system.

Thus, despite the similarity in the general approach about the constituents (colored diquark and triquark) of the pentaquarks, the two model are different in details and lead to different spectra. Both models require estimates of the diquark-size effects on the spectroscopy, a point also discussed later in this paper.

\subsection{Mass predictions for the strange hidden-charm pentaquarks}

Including the $s$-quark, the hidden-charm pentaquark spectrum becomes much richer. Depending on the strange content of the pentaquarks, they can be classified into singly-, doubly-, and triple-strange pentaquarks and their $\mathrm{SU}(3)_{F}$-multiplets are in one to one correspondence with the ordinary and strange baryons [51]. The mass estimates given below assume mass-degenerate $u$ - and $d$-quarks. The constituent masses of the quarks are accepted as specified in subsection 5.1. The diquark masses and spin-spin couplings are not $\mathrm{SU}(3)_{F}$-invariant, and their breaking is taken into account (see tables 6 and 7 ). For the $P$-wave pentaquarks, the orbital, spin-orbit and tensor couplings are assumed equal for all three light quarks and are taken from table 9.

The masses of the singly-strange pentaquarks are presented in tables 10 and 11 and compared with the results of $[51,52]$. The difference between the entries in the two tables lies in the $s$-quark attachment either to the heavy diquark or to the light diquark, i.e., the pentaquark structures are $\left(\bar{c}_{\overline{3}}[c s]_{\overline{3}}\left[q q^{\prime}\right]_{\overline{3}}\right)$ and $\left(\bar{c}_{\overline{3}}[c q]_{\overline{3}}\left[s q^{\prime}\right]_{\overline{3}}\right)$, respectively, with $q^{(\prime)}$ being $u$ - or $d$-quark. One should also remember that the states presented in tables 10 and 11 with the same set of quantum numbers: spin-parity $J^{P}$ and isospin $I$, having strangeness 


\begin{tabular}{|c|c|c|c|c|c|}
\hline$J^{P}$ & This work & refs. $[51,52]$ & $J^{P}$ & This work & refs. $[51,52]$ \\
\hline \multicolumn{3}{|c|}{$S_{l d}=0, L=0$} & \multicolumn{3}{|c|}{$S_{l d}=1, L=1$} \\
\hline \multirow[t]{2}{*}{$1 / 2^{-}$} & $3961 \pm 34$ & $4318 \pm 42$ & \multirow[t]{3}{*}{$1 / 2^{+}$} & $4275 \pm 37$ & $4202 \pm 50$ \\
\hline & $4292 \pm 29$ & $4392 \pm 38$ & & $4341 \pm 37$ & $4406 \pm 44$ \\
\hline $3 / 2^{-}$ & $4362 \pm 29$ & $4365 \pm 55$ & & $4607 \pm 32$ & $4430 \pm 50$ \\
\hline \multicolumn{3}{|c|}{$S_{l d}=1, L=0$} & \multirow{8}{*}{$3 / 2^{+}$} & $4672 \pm 32$ & $4453 \pm 40$ \\
\hline $1 / 2^{-}$ & $4157 \pm 31$ & $4351 \pm 42$ & & $4685 \pm 33$ & $4472 \pm 50$ \\
\hline \multirow{5}{*}{$3 / 2^{-}$} & $4488 \pm 25$ & $4398 \pm 38$ & & $4784 \pm 32$ & $4551 \pm 43$ \\
\hline & $4558 \pm 25$ & $4496 \pm 41$ & & \multicolumn{2}{|l|}{$4319 \pm 37$} \\
\hline & $4157 \pm 31$ & $4304 \pm 55$ & & \multicolumn{2}{|l|}{$4381 \pm 37$} \\
\hline & $4488 \pm 25$ & $4532 \pm 40$ & & \multicolumn{2}{|l|}{$4650 \pm 32$} \\
\hline & $4558 \pm 25$ & $4574 \pm 40$ & & \multicolumn{2}{|l|}{$4712 \pm 32$} \\
\hline $5 / 2^{-}$ & $4558 \pm 25$ & $4641 \pm 40$ & & \multicolumn{2}{|l|}{$4633 \pm 33$} \\
\hline \multicolumn{3}{|c|}{$S_{l d}=0, L=1$} & \multicolumn{3}{|c|}{$4687 \pm 32$} \\
\hline \multirow[t]{3}{*}{$1 / 2^{+}$} & $4161 \pm 39$ & $4262 \pm 63$ & \multirow{3}{*}{$5 / 2^{+}$} & \multicolumn{2}{|l|}{$4778 \pm 32$} \\
\hline & $4492 \pm 35$ & $4373 \pm 44$ & & $4391 \pm 37$ & $4682 \pm 57$ \\
\hline & $4552 \pm 35$ & $4449 \pm 40$ & & $4723 \pm 32$ & $4756 \pm 41$ \\
\hline \multirow[t]{3}{*}{$3 / 2^{+}$} & $4171 \pm 39$ & & & $4723 \pm 32$ & $4910 \pm 44$ \\
\hline & $4503 \pm 35$ & & \multirow{3}{*}{$7 / 2^{+}$} & $4777 \pm 32$ & $4952 \pm 44$ \\
\hline & $4562 \pm 35$ & & & \multicolumn{2}{|l|}{$4794 \pm 32$} \\
\hline $5 / 2^{+}$ & $4579 \pm 35$ & $4742 \pm 57$ & & & \\
\hline
\end{tabular}

Table 10. Masses of the hidden-charm strange pentaquarks (in $\mathrm{MeV}$ ) with a strange-heavy diquark, i.e. having the structure $\left(\bar{c}_{\overline{3}}[c s]_{\overline{3}}\left[q q^{\prime}\right]_{\overline{3}}\right)$, where $q^{(\prime)}$ is $u$ - or $d$-quark, and their comparison with the results presented in $[51,52]$. For the $P$-wave pentaquarks, the values of the orbital, spin-orbit and tensor couplings are taken from table 9.

$S=-1$, are experimentally indistinguishable and, hence, mix with each other. Thus, the mass eigenstates, resulting from this mixing, and the weak interaction eigenstates, in which the decay of the $\Xi_{b}$-baryon would lead dominantly to the states with the internal quantum number $\left(\bar{c}_{\overline{3}}[c q] \overline{3}\left[s q^{\prime}\right]_{\overline{3}}\right)$, are different. In the present analysis, we have not taken into account this mixing.

As for the discovery modes of such pentaquarks in weak decays of bottom baryons, they were studied in [51] in the heavy-quark symmetry limit, assuming $\mathrm{SU}(3)_{F}$-symmetry. Both Cabibbo-allowed $b \rightarrow c \bar{c} s$ (with $\Delta I=0, \Delta S=-1$ ) and Cabibbo-suppressed $b \rightarrow c \bar{c} d$ (with $\Delta I=1 / 2, \Delta S=0$ ) transitions were considered. Among the possible modes of the weakly-decaying $b$-baryons, the most promising modes in searching for the hidden-charm singly-strange pentaquarks at the LHC are: $\Xi_{b}^{-} \rightarrow P_{\Lambda}^{0} K^{-} \rightarrow J / \psi \Lambda^{0} K^{-}$and $\Xi_{b}^{0,-} \rightarrow$ $P_{\Sigma}^{+, 0} K^{-} \rightarrow J / \psi \Sigma^{+, 0} K^{-}$, where the symbol in the subscript on the pentaquarks denotes its light quark content, expressed as the corresponding baryon in the $\mathrm{SU}(3)_{F}$-multiplet. 


\begin{tabular}{|c|c|c|c|c|c|}
\hline$J^{P}$ & This work & refs. $[51,52]$ & $J^{P}$ & This work & refs. $[51,52]$ \\
\hline \multicolumn{3}{|c|}{$S_{l d}=0, L=0$} & \multicolumn{3}{|c|}{$S_{l d}=1, L=1$} \\
\hline \multirow[t]{2}{*}{$1 / 2^{-}$} & $4112 \pm 32$ & $4094 \pm 44$ & \multirow[t]{3}{*}{$1 / 2^{+}$} & $4348 \pm 36$ & $3929 \pm 53$ \\
\hline & $4433 \pm 26$ & $4132 \pm 43$ & & $4414 \pm 36$ & $4183 \pm 45$ \\
\hline $3 / 2^{-}$ & $4523 \pm 26$ & $4172 \pm 47$ & & $4669 \pm 32$ & $4159 \pm 53$ \\
\hline \multicolumn{3}{|c|}{$S_{l d}=1, L=0$} & \multirow{8}{*}{$3 / 2^{+}$} & $4735 \pm 32$ & $4189 \pm 44$ \\
\hline \multirow[t]{3}{*}{$1 / 2^{-}$} & $4230 \pm 30$ & $4128 \pm 44$ & & $4768 \pm 32$ & $4201 \pm 53$ \\
\hline & $4551 \pm 25$ & $4134 \pm 42$ & & $4867 \pm 32$ & $4275 \pm 45$ \\
\hline & $4641 \pm 25$ & $4220 \pm 43$ & & $4392 \pm 36$ & \\
\hline \multirow[t]{3}{*}{$3 / 2^{-}$} & $4230 \pm 30$ & $4031 \pm 43$ & & $4454 \pm 36$ & \\
\hline & $4551 \pm 25$ & $4262 \pm 43$ & & $4713 \pm 32$ & \\
\hline & $4641 \pm 25$ & $4303 \pm 43$ & & $4775 \pm 32$ & \\
\hline $5 / 2^{-}$ & $4641 \pm 25$ & $4370 \pm 43$ & & $4716 \pm 32$ & \\
\hline \multicolumn{3}{|c|}{$S_{l d}=0, L=1$} & & $4770 \pm 32$ & \\
\hline \multirow[t]{3}{*}{$1 / 2^{+}$} & $4312 \pm 37$ & $4069 \pm 56$ & & $4861 \pm 32$ & \\
\hline & $4633 \pm 33$ & $4149 \pm 45$ & $5 / 2^{+}$ & $4465 \pm 36$ & $4409 \pm 47$ \\
\hline & $4713 \pm 33$ & $4187 \pm 44$ & & $4786 \pm 32$ & $4486 \pm 45$ \\
\hline \multirow[t]{3}{*}{$3 / 2^{+}$} & $4323 \pm 37$ & & & $4806 \pm 32$ & $4639 \pm 47$ \\
\hline & $4643 \pm 33$ & & & $4860 \pm 32$ & $4681 \pm 47$ \\
\hline & $4723 \pm 33$ & & $7 / 2^{+}$ & $4877 \pm 32$ & \\
\hline $5 / 2^{+}$ & $4740 \pm 33$ & $4549 \pm 51$ & & & \\
\hline
\end{tabular}

Table 11. Masses of the hidden-charm strange pentaquarks (in $\mathrm{MeV}$ ) with a strange-light diquark, i.e. having the structure $\left(\bar{c}_{\overline{3}}[c q]_{\overline{3}}\left[s q^{\prime}\right]_{\overline{3}}\right)$, where $q^{(\prime)}$ is $u$ - or $d$-quark, and their comparison with the results presented in $[51,52]$. For the $P$-wave pentaquarks, the values of the orbital, spin-orbit and tensor couplings are taken from table 9 .

Assuming a similarity in the unflavored and strange pentaquark production, we anticipate the observation of the strange partners of the known pentaquarks. We have marked the masses of these states by enclosing them in solid boxes in table 11. The threshold for the strange pentaquarks in the $P_{\Lambda}^{0} \rightarrow J / \psi \Lambda$ decay mode is $M_{J / \psi \Lambda}^{\mathrm{thr}}=m_{J / \psi}+m_{\Lambda}=$ $4212.58 \mathrm{MeV}$ [3]. With the masses given in table 11, there is only one state with the "good" light diquark $\left(S_{l d}=0\right)$, the $J^{P}=1 / 2^{-}$with the mass $M=(4112 \pm 32) \mathrm{MeV}$, which lies $100 \mathrm{MeV}$ below the $M_{J / \psi \Lambda}^{\mathrm{thr}}$ threshold. This will result into a state narrower than one should expect due to a strong decay, with the $c \bar{c}$-pair annihilating into light hadrons or a lepton pair. This makes a good case for a search of narrow $\Lambda \mu^{+} \mu^{-}$(and $\Lambda e^{+} e^{-}$) resonant structure. The thresholds in other two modes $P_{\Sigma}^{+, 0} \rightarrow J / \psi \Sigma^{+, 0}$ are higher: $M_{J / \psi \Sigma^{+}}^{\mathrm{thr}}=m_{J / \psi}+m_{\Sigma^{+}}=4286.27 \mathrm{MeV}$ and $M_{J / \psi \Sigma^{0}}^{\mathrm{thr}}=m_{J / \psi}+m_{\Sigma^{0}}=4289.54 \mathrm{MeV}$ [3], and there are two mass-degenerate states with the mass $M=4230 \mathrm{MeV}$ and spin-parities $J^{P}=1 / 2^{-}$and $J^{P}=3 / 2^{-}$which are below these thresholds. One should remember that 


\begin{tabular}{|c|c|c|c|c|c|}
\hline$J^{P}$ & This work & refs. $[51,52]$ & $J^{P}$ & This work & refs. $[51,52]$ \\
\hline \multicolumn{3}{|c|}{$S_{l d}=0, L=0$} & \multicolumn{3}{|c|}{$S_{l d}=1, L=1$} \\
\hline \multirow[t]{2}{*}{$1 / 2^{-}$} & $4243 \pm 32$ & $4326 \pm 44$ & \multirow[t]{3}{*}{$1 / 2^{+}$} & $4480 \pm 37$ & $4161 \pm 53$ \\
\hline & $4575 \pm 26$ & $4364 \pm 43$ & & $4545 \pm 37$ & $4415 \pm 45$ \\
\hline $3 / 2^{-}$ & $4644 \pm 26$ & $4404 \pm 47$ & & $4811 \pm 32$ & $4391 \pm 53$ \\
\hline \multicolumn{3}{|c|}{$S_{l d}=1, L=0$} & \multirow{8}{*}{$3 / 2^{+}$} & $4877 \pm 32$ & $4421 \pm 44$ \\
\hline $1 / 2^{-}$ & $4361 \pm 31$ & $4360 \pm 43$ & & $4890 \pm 32$ & $4433 \pm 52$ \\
\hline \multirow{5}{*}{$3 / 2^{-}$} & $4693 \pm 25$ & $4366 \pm 43$ & & $4989 \pm 32$ & $4507 \pm 45$ \\
\hline & $4762 \pm 25$ & $4452 \pm 43$ & & \multicolumn{2}{|l|}{$4523 \pm 37$} \\
\hline & $4361 \pm 31$ & $4263 \pm 43$ & & \multicolumn{2}{|l|}{$4585 \pm 37$} \\
\hline & $4693 \pm 25$ & $4494 \pm 43$ & & \multicolumn{2}{|l|}{$4855 \pm 32$} \\
\hline & $4762 \pm 25$ & $4535 \pm 43$ & & \multicolumn{2}{|l|}{$4917 \pm 32$} \\
\hline $5 / 2^{-}$ & $4762 \pm 25$ & $4602 \pm 43$ & & \multicolumn{2}{|l|}{$4837 \pm 32$} \\
\hline \multicolumn{3}{|c|}{$S_{l d}=0, L=1$} & \multicolumn{3}{|c|}{$4892 \pm 32$} \\
\hline \multirow[t]{3}{*}{$1 / 2^{+}$} & $4443 \pm 38$ & $4301 \pm 56$ & \multirow{3}{*}{$5 / 2^{+}$} & \multicolumn{2}{|l|}{$4982 \pm 32$} \\
\hline & $4775 \pm 33$ & $4381 \pm 45$ & & $4596 \pm 37$ & $4641 \pm 47$ \\
\hline & $4834 \pm 33$ & $4419 \pm 44$ & & $4928 \pm 32$ & $4718 \pm 45$ \\
\hline \multirow[t]{3}{*}{$3 / 2^{+}$} & $4454 \pm 38$ & & & $4927 \pm 32$ & $4871 \pm 47$ \\
\hline & $4785 \pm 33$ & & \multirow{3}{*}{$7 / 2^{+}$} & $4982 \pm 32$ & $4913 \pm 47$ \\
\hline & $4844 \pm 33$ & & & \multicolumn{2}{|l|}{$4998 \pm 32$} \\
\hline $5 / 2^{+}$ & $4861 \pm 33$ & $4781 \pm 51$ & & & \\
\hline
\end{tabular}

Table 12. Masses of the hidden-charm doubly-strange pentaquarks (in $\mathrm{MeV}$ ) with both the heavy and light strange diquarks, i.e. having the structure $\left(\bar{c}_{\overline{3}}[c s]_{\overline{3}}[s q]_{\overline{3}}\right)$, where $q$ is $u$ - or $d$-quark, and their comparison with the results presented in $[51,52]$. For the $P$-wave pentaquarks, the values of the orbital, spin-orbit and tensor couplings are taken from table 9 .

these states contain the "bad" light diquark and their production in the $\Xi_{b}$-baryon decays is suppressed by heavy quark symmetry.

The masses of doubly-strange pentaquarks are presented in tables 12 and 13 and compared with the results of $[51,52]$. The difference between the entries in these tables is as follows: the states from table 12 contain both the strange heavy diquark and the strange light diquark, while those from table 13 have hidden-charm unflavored triquark and doublystrange light diquark, i.e., the pentaquark structures are $\left(\bar{c}_{\overline{3}}[c s]_{\overline{3}}[s q]_{\overline{3}}\right)$ and $\left(\bar{c}_{\overline{3}}[c q]_{\overline{3}}\{s s\}_{\overline{3}}\right)$, respectively, with $q$ being $u$ - or $d$-quark. The number of states with the doubly-strange light diquark is smaller ${ }^{5}$ because such a diquark, being flavor-symmetric, can be only the

\footnotetext{
${ }^{5}$ In refs. [51, 52] similar analysis was done for the hidden-charm doubly-strange pentaquarks and mass predictions for the states with the doubly-strange "good" light diquark were presented erroneously. The corresponding entries should be excluded from tables V and VI (the last values in the columns entitled $\mathcal{P}_{X_{2}}$ and $\mathcal{P}_{Y_{2}}$, respectively) in [51] and from tables $\mathrm{V}$ (the last values in the columns entitled $\mathcal{P}_{X_{1}}$ and $\mathcal{P}_{X_{3}}$ ), VI (similar values in the columns $\mathcal{P}_{Y_{1}}$ and $\mathcal{P}_{Y_{3}}$ ), and VII (the last value in the columns entitled $\mathcal{P}_{Y_{7}}$ ) in [52].
} 


\begin{tabular}{|c|c|c|c|c|c|}
\hline$J^{P}$ & This work & refs. $[51,52]$ & $J^{P}$ & This work & refs. $[51,52]$ \\
\hline \multicolumn{3}{|c|}{$S_{l d}=1, L=0$} & \multicolumn{3}{|c|}{$S_{l d}=1, L=1$} \\
\hline \multirow{3}{*}{$1 / 2^{-}$} & $4511 \pm 30$ & $4598 \pm 44$ & \multirow[t]{7}{*}{$3 / 2^{+}$} & $4673 \pm 36$ & \\
\hline & $4832 \pm 25$ & $4666 \pm 43$ & & $4735 \pm 36$ & \\
\hline & $4922 \pm 25$ & $4775 \pm 44$ & & $4994 \pm 32$ & \\
\hline \multirow[t]{3}{*}{$3 / 2^{-}$} & $4511 \pm 30$ & $4577 \pm 43$ & & $5056 \pm 32$ & \\
\hline & $4832 \pm 25$ & $4810 \pm 43$ & & $4997 \pm 32$ & \\
\hline & $4922 \pm 25$ & $4851 \pm 43$ & & $5051 \pm 32$ & \\
\hline $5 / 2^{-}$ & $4922 \pm 25$ & $4918 \pm 47$ & & $5142 \pm 32$ & \\
\hline \multirow{7}{*}{$1 / 2^{+}$} & \multicolumn{2}{|c|}{$S_{l d}=1, L=1$} & \multirow[t]{4}{*}{$5 / 2^{+}$} & $4746 \pm 36$ & $4954 \pm 47$ \\
\hline & $4629 \pm 36$ & $4474 \pm 53$ & & $5067 \pm 32$ & $5033 \pm 47$ \\
\hline & $4695 \pm 36$ & $4653 \pm 45$ & & $5087 \pm 32$ & $5187 \pm 47$ \\
\hline & $4950 \pm 32$ & $4707 \pm 53$ & & $5141 \pm 32$ & $5228 \pm 47$ \\
\hline & $5016 \pm 32$ & $4721 \pm 44$ & \multirow[t]{3}{*}{$7 / 2^{+}$} & $5158 \pm 32$ & \\
\hline & $5049 \pm 32$ & $4748 \pm 52$ & & & \\
\hline & $5148 \pm 32$ & $4830 \pm 45$ & & & \\
\hline
\end{tabular}

Table 13. Masses of the hidden-charm doubly-strange pentaquarks (in MeV) with the doublystrange light diquark, i.e. having the structure $\left(\bar{c}_{\overline{3}}[c q]_{\overline{3}}\{s s\}_{\overline{3}}\right)$, where $q$ is $u$-or $d$-quark and curly brackets denote the spin-1 light diquark, and their comparison with the results presented in [51, 52]. For the $P$-wave pentaquarks, the values of the orbital, spin-orbit and tensor couplings are taken from table 9 .

"bad" diquark with the spin $S_{l d}=1$. So, this means that the pentaquarks with the "good" light diquark from table 12 remain unmixed while the states with the "bad" light diquark mix, in general, with the corresponding states (with the same set of quantum numbers: spin-parity $J^{P}$ and isospin $I$ ) from table 13. In this paper, we neglect this mixing.

Based on the analysis in [51], we specify the most promising decay mode of the $\Omega_{b^{-}}$ baryon for searching of hidden-charm doubly-strange pentaquarks at the LHC as $\Omega_{b}^{-} \rightarrow$ $P_{\Xi_{10}}^{0} K^{-} \rightarrow J / \psi \Xi^{\prime 0} K^{-}$. The threshold for the doubly-strange pentaquarks in the $P_{\Xi_{10}}^{0} \rightarrow$ $J / \psi \Xi^{\prime 0}$ decay mode is $M_{J / \psi \Xi^{\prime 0}}^{\mathrm{thr}}=m_{J / \psi}+m_{\Xi^{\prime 0}}=(4628.70 \pm 0.32) \mathrm{MeV}$ [3]. With the masses given in table 13 , there are two mass degenerate states, having $J^{P}=1 / 2^{-}$and $J^{P}=3 / 2^{-}$, with the mass $M=4511 \mathrm{MeV}$, which lie below the $M_{J / \psi \Xi^{\prime 0}}^{\mathrm{thr}}$ threshold. Also, the third orbitally-excited state with the spin-parity $J^{P}=1 / 2^{+}$and the mass $M=4629 \mathrm{MeV}$ could also lie below this threshold. All the other states presented in table 13 can be produced in the $\Omega_{b}$-baryon decays and their observation will herald the era of the as yet unknown field of doubly-strange pentaquarks.

The masses of the triple-strange pentaquarks are presented in table 14. As these pentaquarks do not contain $u$ - or $d$-quarks, they are isospin singlets. All these states have the doubly-strange "bad" light diquark only, i.e., the pentaquark structure is $\left(\bar{c}_{\overline{3}}[c s] \overline{3}\{s s\}_{\overline{3}}\right)$. They can be produced at the LHC in the weak decays of the $\Omega_{b}$-baryons, in particular, 


\begin{tabular}{|c|c|c|c|}
\hline$J^{P}$ & Mass & $J^{P}$ & Mass \\
\hline \multicolumn{2}{|c|}{$S_{l d}=1, L=0$} & \multicolumn{2}{|c|}{$S_{l d}=1, L=1$} \\
\hline \multirow[t]{3}{*}{$1 / 2^{-}$} & $4642 \pm 31$ & \multirow[t]{6}{*}{$3 / 2^{+}$} & $4804 \pm 37$ \\
\hline & $4974 \pm 25$ & & $4866 \pm 37$ \\
\hline & $5043 \pm 25$ & & $5136 \pm 32$ \\
\hline \multirow[t]{3}{*}{$3 / 2^{-}$} & $4642 \pm 31$ & & $5198 \pm 32$ \\
\hline & $4974 \pm 25$ & & $5118 \pm 32$ \\
\hline & $5043 \pm 25$ & & $5173 \pm 32$ \\
\hline $5 / 2^{-}$ & $5043 \pm 25$ & \multirow{5}{*}{$5 / 2^{+}$} & $5263 \pm 32$ \\
\hline \multicolumn{2}{|c|}{$S_{l d}=1, L=1$} & & $4877 \pm 37$ \\
\hline \multirow[t]{6}{*}{$1 / 2^{+}$} & $4761 \pm 37$ & & $5209 \pm 32$ \\
\hline & $4826 \pm 37$ & & $5208 \pm 32$ \\
\hline & $5092 \pm 32$ & & $5263 \pm 32$ \\
\hline & $5158 \pm 32$ & \multirow[t]{3}{*}{$7 / 2^{+}$} & $5279 \pm 32$ \\
\hline & $5171 \pm 32$ & & \\
\hline & $5270 \pm 32$ & & \\
\hline
\end{tabular}

Table 14. Masses of the hidden-charm triple-strange pentaquarks (in $\mathrm{MeV}$ ) containing the strange heavy diquark and doubly-strange light diquark, i.e. having the structure $\left(\bar{c}_{\overline{3}}[c s]_{\overline{3}}\{s s\}_{\overline{3}}\right)$, where curly brackets denote the spin- 1 light diquark. For the $P$-wave pentaquarks, the values of the orbital, spin-orbit and tensor couplings are taken from table 9.

due to the $b \rightarrow c \bar{c} s$ transition which gives rise to the quasi-two-body decay mode $\Omega_{b}^{-} \rightarrow$ $P_{\Omega_{10}}^{-} \phi \rightarrow J / \psi \Omega^{-} \phi$. The threshold for the triple-strange pentaquarks in the $P_{\Omega_{10}}^{-} \rightarrow$ $J / \psi \Omega^{-}$decay is $M_{J / \psi \Omega^{-}}^{\mathrm{thr}}=m_{J / \psi}+m_{\Omega^{-}}=(4769.35 \pm 0.29) \mathrm{MeV}$ [3]. With the masses given in table 14 , there are two mass degenerate states, having $J^{P}=1 / 2^{-}$and $J^{P}=3 / 2^{-}$, with the mass $M=(4642 \pm 31) \mathrm{MeV}$, which lie $\sim 100 \mathrm{MeV}$ below the $M_{J / \psi \Omega^{-}}^{\mathrm{thr}}$ threshold. Also, the third orbitally-excited state with the spin-parity $J^{P}=1 / 2^{+}$and the mass $M=$ $(4761 \pm 37) \mathrm{MeV}$ could also lie below this threshold. All the other states presented in table 14 can be produced in the $\Omega_{b}$-baryon decays. The other possibility is to produce such pentaquarks promptly, but their production is highly suppressed due to their triple-strange content.

\section{Pentaquark decay widths}

In the compact diquark picture, the quarks in a diquark are bound and not free. In the present context it means that there is a barrier (or bound-state effect) which reduces the probability of the $\bar{c}$-quark and the charm quark in the [uc]-diquark to form a charmonium state. This is seen also in the decays of the $X, Y, Z$ states, which are tetraquark candidates in the compact diquark picture $[48,54,66-69]$. In the case of the hidden-charm tetraquarks, it is the radius of the tetraquark $R_{4 q} \sim 1-2 \mathrm{fm}$ (or, perhaps somewhat larger) compared to the size of the compact heavy-light diquark, typically $R_{Q q} \sim 0.5 \mathrm{fm}$, which acts as a 
barrier to form a $J / \psi$ - or $\eta_{c}$-meson. For example, it is argued in [66], that the relative ratio $\lambda$ is expected to be $\lambda \equiv R_{4 q} / R_{Q q} \geq 3$. At long distance (but within the confined tetraquark radius), the potential is attractive, which changes as the internal structure of the diquark (and anti-diquark) gets resolved. The tunneling probability depends on the mass of the quark, with the probability exponentially suppressed the heavier the quark is. This can be expressed through the semi-classical approximation of the tunneling amplitude $A_{M} \sim e^{-\sqrt{2 M E}} R_{4 q}$, where $M$ is the quark mass and the energy $E$ is typically $100 \mathrm{MeV}$ [66]. This suppresses the formation of the charmonium states, leading to a much reduced $P_{c} \rightarrow$ $J / \psi p$ decay width. In all these cases, the size of the diquark $R_{Q q}$ plays an important role in reducing the decay widths, though the extent of this suppression may be different in the tetraquark and pentaquark cases. To work it out requires a dynamical theory, which is currently not at hand. As some of the states are orbitally excited states, anticipated in the compact diquark picture, the decay widths are further reduced due to the angular momentum barrier. Diquark-size effects are also present in the tetraquark and pentaquark mass estimates in the diquark model, and they have been calculated in a particular case, namely double-bottom tetraquark [70].

For four quarks $\left(q, q^{\prime}, q^{\prime \prime}\right.$ and $\left.c\right)$ and the charm antiquark, present in a pentaquark $P_{c}$, there are different alternatives for clustering in color neutral states. The first possibility is the triquark-diquark alternative:

$$
\begin{aligned}
& \Psi_{1}^{D}=\frac{1}{\sqrt{3}}\left[\frac{1}{\sqrt{2}} \epsilon_{i j k} \bar{c}^{i}\left[\frac{1}{\sqrt{2}} \epsilon^{j l m} c_{l} q_{m}\right]\right]\left[\frac{1}{\sqrt{2}} \epsilon^{k n p} q_{n}^{\prime} q_{p}^{\prime \prime}\right] \equiv[\bar{c}[c q]]\left[q^{\prime} q^{\prime \prime}\right] \\
& \Psi_{2}^{D}=\frac{1}{\sqrt{3}}\left[\frac{1}{\sqrt{2}} \epsilon_{i k j} \bar{c}^{i}\left[\frac{1}{\sqrt{2}} \epsilon^{k n p} q_{n}^{\prime} q_{p}^{\prime \prime}\right]\right]\left[\frac{1}{\sqrt{2}} \epsilon^{j l m} c_{l} q_{m}\right] \equiv\left[\bar{c}\left[q^{\prime} q^{\prime \prime}\right]\right][c q] .
\end{aligned}
$$

From the color algebra point of view, these states are related, $\Psi_{2}^{D}=-\Psi_{1}^{D}$, but other internal dynamical properties can be different. The square parenthesis on the r.h.s. of the second equality in each equation denote color antisymmetrization of the constituents. The color connection of the quarks in $\Psi_{1}^{D}$ is assumed in the present paper, while the $\Psi_{2}^{D}$ color structure is employed in the dynamical diquark model of multiquark exotic hadrons [50, 54, 68]. The other color-singlet combinations are the meson-baryon alternatives:

$$
\begin{aligned}
& \Psi_{1}^{H}=\left(\frac{1}{\sqrt{3}} \bar{c}^{i} c_{i}\right)\left[\frac{1}{\sqrt{6}} \epsilon^{j k l} q_{j} q_{k}^{\prime} q_{l}^{\prime \prime}\right] \equiv(\bar{c} c)\left[q q^{\prime} q^{\prime \prime}\right], \\
& \Psi_{2}^{H}=\left(\frac{1}{\sqrt{3}} \bar{c}^{i} q_{i}\right)\left[\frac{1}{\sqrt{6}} \epsilon^{j k l} c_{j} q_{k}^{\prime} q_{l}^{\prime \prime}\right] \equiv(\bar{c} q)\left[c q^{\prime} q^{\prime \prime}\right], \\
& \Psi_{3}^{H}=\left(\frac{1}{\sqrt{3}} \bar{c}^{i} q_{i}^{\prime}\right)\left[\frac{1}{\sqrt{6}} \epsilon^{j k l} c_{j} q_{k} q_{l}^{\prime \prime}\right] \equiv\left(\bar{c} q^{\prime}\right)\left[c q q^{\prime \prime}\right], \\
& \Psi_{4}^{H}=\left(\frac{1}{\sqrt{3}} \bar{c}^{i} q_{i}^{\prime \prime}\right)\left[\frac{1}{\sqrt{6}} \epsilon^{j k l} c_{j} q_{k} q_{l}^{\prime}\right] \equiv\left(\bar{c} q^{\prime \prime}\right)\left[c q q^{\prime}\right] .
\end{aligned}
$$

Among these four combinations, only $\Psi_{1}^{H}$ and $\Psi_{2}^{H}$ satisfy the heavy-quark-symmetry condition, which implies that the light $\left[q^{\prime} q^{\prime \prime}\right]$-diquark is transmitted intact, retaining its spin quantum number, from the $b$-baryon decays to the pentaquark. 
It is possible to reduce the states in the color configurations (6.1) or (6.2) to a linear combination of the states in the set (6.3)-(6.6). Keeping the color of the light diquark unchanged, the convolution of two Levi-Civita tensors entering the triquark in (6.1) gives the following decomposition:

$$
\Psi_{1}^{D}=-\frac{\sqrt{3}}{2}\left[\Psi_{1}^{H}+\Psi_{2}^{H}\right]
$$

as mentioned earlier. However, a color reconnection is not enough to reexpress a pentaquark operator as a direct product of the meson and baryon operators. As hadrons are determined by their spin-parities, one should also project spins of the quarks and diquarks onto definite hadronic spin states. This requires to know the Dirac structure of the pentaquark operators to undertake the Fierz transformations in the Dirac space. This is a rather involved problem, already for the $S$-wave pentaquarks, and requires a dedicated study, to which we hope to return in a future publication.

We exemplify it here by considering the three $S$-wave pentaquarks from table 2 with a light "good" diquark, following similar considerations for the hidden-charm tetraquarks [66]. The heavy diquark in them, being also in $S$-wave, can have both spins, $S_{h d}=0$ and $S_{h d}=1$. We further simplify the problem by assuming that the charm quark and antiquark are non-relativistic particles, keeping them as point-like objects at different positions in the Minkowski space-time. The diquark-diquark-antiquark operators of the first state from table 2, containing the heavy-diquark with $S_{h d}=0$, after color reconnection (the spin structure is determined by eq. (6.1)) and making the color indices explicit, are as follows:

$$
\begin{aligned}
& \Psi_{1}^{H(1)}(x, y, z)=\frac{1}{3}\left(\tilde{c}^{i}(x) \sigma_{2}\right)\left(c_{i}(y) \sigma_{2} q_{k}(y)\right) d_{0}^{k}(z), \\
& \Psi_{2}^{H(1)}(x, y, z)=\frac{1}{3}\left(\tilde{c}^{i}(x) \sigma_{2}\right)\left(c_{k}(y) \sigma_{2} q_{i}(y)\right) d_{0}^{k}(z),
\end{aligned}
$$

where $\tilde{c}(x), c(y)$ and $q(y)$ are the charm-antiquark, charm-quark and light-quark spinors in the non-relativistic limit, respectively, $i$ and $k$ are the color indices, the light diquark operator is denoted as $d_{0}(z)$, and the usual notation for the product of matrices is employed. With the help of the Fierz transform for the Pauli matrices [15]:

$$
\left(\sigma_{2}\right)_{\beta}^{\alpha}\left(\sigma_{2}\right)_{\delta}^{\gamma}=\frac{1}{2}\left[\left(\sigma_{2}\right)_{\delta}^{\alpha}\left(\sigma_{2}\right)_{\beta}^{\gamma}+\left(\sigma_{2} \sigma_{a}\right)_{\delta}^{\alpha}\left(\sigma_{2} \sigma_{a}\right)_{\beta}^{\gamma}\right],
$$

we reduce the states (6.8) and (6.9) to the forms:

$$
\begin{aligned}
& \Psi_{1}^{H(1)}(x, y, z)=-\frac{1}{2}\left[\left(\tilde{c}(x) \sigma_{2} c(y)\right)\left(q(y) \sigma_{2} d_{0}(z)\right)+\left(\tilde{c}(x) \sigma_{2} \boldsymbol{\sigma} c(y)\right)\left(q(y) \sigma_{2} \boldsymbol{\sigma} d_{0}(z)\right)\right], \\
& \Psi_{2}^{H(1)}(x, y, z)=-\frac{1}{2}\left[\left(\tilde{c}(x) \sigma_{2} q(y)\right)\left(c(y) \sigma_{2} d_{0}(z)\right)+\left(\tilde{c}(x) \sigma_{2} \boldsymbol{\sigma} q(y)\right)\left(c(y) \sigma_{2} \boldsymbol{\sigma} d_{0}(z)\right)\right]
\end{aligned}
$$

where the quark-antiquark and quark-diquark combinations are properly normalized in the color space.

The transition to hadrons needs specifying the flavors of all the quarks. If, as an example, we consider pentaquarks with the same quark content as the observed ones, this 
immediately specifies $q=u$ and $d_{0}=\left[u C \gamma_{5} d\right]$, being the color-antitriplet scalar state. ${ }^{6}$ Neglecting the position dependence of the operators, the quark operators (6.11) and (6.12) can be rewritten in terms of the hadrons:

$$
\begin{aligned}
& \Psi_{1}^{H(1)}=-\frac{i}{\sqrt{2}} p\left[a \eta_{c}+b(\boldsymbol{\sigma} \mathbf{J} / \boldsymbol{\psi})\right], \\
& \Psi_{2}^{H(1)}=-\frac{i}{\sqrt{2}} \Lambda_{c}^{+}\left[A \bar{D}^{0}+B\left(\boldsymbol{\sigma} \overline{\mathbf{D}}^{* \mathbf{0}}\right)\right],
\end{aligned}
$$

where $A$ and $B$ ( $a$ and $b$ ) are non-perturbative coefficients associated with different barrier penetration amplitudes for different light-quark (heavy-quark) spin configurations. They are equal in the limit of naive Fierz coupling. As mentioned in subsection 5.2, the predicted mass of this state, $M=(3830 \pm 34) \mathrm{MeV}$, is below the $J / \psi p$ and $\eta_{c} p$ thresholds, so its decay to these states is forbidden by phase space, and it will decay through the charmanticharm pair annihilation, having a width much smaller than the widths of the newly observed pentaquarks in table 1 . The decays of this pentaquark to the $\Lambda_{c}^{+} \bar{D}^{0}$ and $\Lambda_{c}^{+} \bar{D}^{* 0}$ final states are also forbidden by phase space, as these channels have higher thresholds than the proton-charmonium modes.

Along similar lines, assigning $q=s$, but keeping the light diquark $d_{0}$ as above, we get the first state with the internal spins $S_{h d}=S_{l d}=0$ from table 11. In terms of the physical hadrons, the quark operators (6.11) and (6.12) can be written as follows:

$$
\begin{aligned}
& \Psi_{1}^{H(1)}=-\frac{i}{\sqrt{2}} \Lambda\left[a_{s} \eta_{c}+b_{s}(\boldsymbol{\sigma} \mathbf{J} / \boldsymbol{\psi})\right], \\
& \Psi_{2}^{H(1)}=-\frac{i}{\sqrt{2}} \Lambda_{c}^{+}\left[A_{s} \bar{D}_{s}^{0}+B_{s}\left(\boldsymbol{\sigma} \overline{\mathbf{D}}_{\mathbf{s}}^{* \mathbf{0}}\right)\right],
\end{aligned}
$$

where $A_{s}$ and $B_{s}\left(a_{s}\right.$ and $\left.b_{s}\right)$ are non-perturbative coefficients associated with barrier penetration amplitudes. The predicted mass of this state, $M=(4112 \pm 32) \mathrm{MeV}$, (see table 11 in subsection 5.4) is below the $J / \psi \Lambda$ and $\eta_{c} \Lambda$ thresholds, so its decay to these states is forbidden by phase space and it will decay through the charm-anticharm pair annihilation. As the thresholds of $\Lambda_{c}^{+} \bar{D}_{s}^{0}$ and $\Lambda_{c}^{+} \bar{D}_{s}^{* 0}$ are higher, the decays of this pentaquark to these final states are also forbidden by phase space.

In the same approximation, the diquark-diquark-antiquark operators of the other states from table 2, containing the heavy-diquark with $S_{h d}=1$, after color reconnection (the spin structure is determined by eq. (6.1)) and making the color indices explicit, are as follows:

$$
\begin{aligned}
& \Psi_{1}^{H(2)}(x, y, z)=\frac{1}{3}\left(\tilde{c}^{i}(x) \sigma_{2}\right)\left(c_{i}(y) \sigma_{2} \boldsymbol{\sigma} q_{k}(y)\right) d_{0}^{k}(z), \\
& \Psi_{2}^{H(2)}(x, y, z)=\frac{1}{3}\left(\tilde{c}^{i}(x) \sigma_{2}\right)\left(c_{k}(y) \sigma_{2} \boldsymbol{\sigma} q_{i}(y)\right) d_{0}^{k}(z) .
\end{aligned}
$$

These operators, being the direct product of a spinor and a vector, are required to be decomposed into two irreducible representations, corresponding to the spin $J=1 / 2$ and $J=3 / 2$ pentaquarks. The projection onto these states is done after Fierz transform is

\footnotetext{
${ }^{6}$ In the diquark, operator $C$ is the charge-conjugation matrix.
} 
performed, for which one uses the relation [15]:

$$
\left(\sigma_{2}\right)_{\beta}^{\alpha}\left(\sigma_{2} \sigma_{a}\right)_{\delta}^{\gamma}=\frac{1}{2}\left[\left(\sigma_{2}\right)_{\delta}^{\alpha}\left(\sigma_{2} \sigma_{a}\right)_{\beta}^{\gamma}+\left(\sigma_{2} \sigma_{a}\right)_{\delta}^{\alpha}\left(\sigma_{2}\right)_{\beta}^{\gamma}+i \varepsilon_{a b c}\left(\sigma_{2} \sigma_{b}\right)_{\delta}^{\alpha}\left(\sigma_{2} \sigma_{c}\right)_{\beta}^{\gamma}\right] .
$$

With this, the states (6.17) and (6.18) are reduced to:

$$
\begin{aligned}
\mathbf{\Psi}_{1}^{H(2)}(x, y, z)= & \frac{1}{2}\left\{\left(\tilde{c}(x) \sigma_{2} \boldsymbol{\sigma} c(y)\right)\left(q(y) \sigma_{2} d_{0}(z)\right)+\left(\tilde{c}(x) \sigma_{2} c(y)\right)\left(q(y) \sigma_{2} \boldsymbol{\sigma} d_{0}(z)\right)\right. \\
& \left.+i\left[\left(\tilde{c}(x) \sigma_{2} \boldsymbol{\sigma} c(y)\right) \times\left(q(y) \sigma_{2} \boldsymbol{\sigma} d_{0}(z)\right)\right]\right\} \\
\mathbf{\Psi}_{2}^{H(2)}(x, y, z)= & -\frac{1}{2}\left\{\left(\tilde{c}(x) \sigma_{2} \boldsymbol{\sigma} q(y)\right)\left(c(y) \sigma_{2} d_{0}(z)\right)+\left(\tilde{c}(x) \sigma_{2} q(y)\right)\left(c(y) \sigma_{2} \boldsymbol{\sigma} d_{0}(z)\right)\right. \\
& \left.+i\left[\left(\tilde{c}(x) \sigma_{2} \boldsymbol{\sigma} q(y)\right) \times\left(c(y) \sigma_{2} \boldsymbol{\sigma} d_{0}(z)\right)\right]\right\}
\end{aligned}
$$

where the quark-antiquark and quark-diquark combinations are properly normalized in the color space.

Neglecting as above the position dependence, the quark operators (6.20) and (6.21) of the pentaquarks with the light quark $q=u$ and light diquark $d_{0}=\left[u C \gamma_{5} d\right]$ can be rewritten in terms of the hadronic states:

$$
\begin{aligned}
& \boldsymbol{\Psi}_{1}^{H(2)}=\frac{i}{\sqrt{2}} p\left\{a^{\prime} \eta_{c} \boldsymbol{\sigma}+b^{\prime} \mathbf{J} / \boldsymbol{\psi}-i c^{\prime}[\boldsymbol{\sigma} \times \mathbf{J} / \boldsymbol{\psi}]\right\}, \\
& \boldsymbol{\Psi}_{2}^{H(2)}=-\frac{i}{\sqrt{2}} \Lambda_{c}^{+}\left\{A^{\prime} \bar{D}^{0} \boldsymbol{\sigma}+B^{\prime} \overline{\mathbf{D}}^{* \mathbf{0}}-i C^{\prime}\left[\boldsymbol{\sigma} \times \overline{\mathbf{D}}^{* \mathbf{0}}\right]\right\},
\end{aligned}
$$

where $A^{\prime}, B^{\prime}$ and $C^{\prime}\left(a^{\prime}, b^{\prime}\right.$ and $\left.c^{\prime}\right)$ are non-perturbative coefficients associated with different barrier penetration amplitudes. They are equal in the limit of naive Fierz coupling. These operators are the linear combination of two operators corresponding to baryons with spins $J=1 / 2$ and $J=3 / 2$. They can be separated by using the equation:

$$
\boldsymbol{\Psi}_{1,2}^{H(2)}=\left[\boldsymbol{\Psi}_{1,2}^{H(2)}-\frac{1}{3}\left(\boldsymbol{\Psi}_{1,2}^{H(2)} \boldsymbol{\sigma}\right) \boldsymbol{\sigma}\right]+\frac{1}{3}\left(\boldsymbol{\Psi}_{1,2}^{H(2)} \boldsymbol{\sigma}\right) \boldsymbol{\sigma}=\boldsymbol{\Psi}_{1,2}^{H(3 / 2)}+\Psi_{1,2}^{H(1 / 2)} \boldsymbol{\sigma}
$$

with the following result:

$$
\begin{aligned}
& \Psi_{1}^{H(1 / 2)}=\frac{i}{\sqrt{2}} p\left\{a^{\prime} \eta_{c}+\frac{b^{\prime}}{3}(\boldsymbol{\sigma} \mathbf{J} / \boldsymbol{\psi})\right\}, \\
& \Psi_{1}^{H(3 / 2)}=\frac{i \sqrt{2}}{3} p\left\{b^{\prime} \mathbf{J} / \boldsymbol{\psi}-2 i c^{\prime}[\boldsymbol{\sigma} \times \mathbf{J} / \boldsymbol{\psi}]\right\}, \\
& \Psi_{2}^{H(1 / 2)}=-\frac{i}{\sqrt{2}} \Lambda_{c}^{+}\left\{A^{\prime} \bar{D}^{0}+\frac{B^{\prime}}{3}\left(\boldsymbol{\sigma} \overline{\mathbf{D}}^{* \mathbf{0}}\right)\right\}, \\
& \Psi_{2}^{H(3 / 2)}=-\frac{i \sqrt{2}}{3} \Lambda_{c}^{+}\left\{B^{\prime} \overline{\mathbf{D}}^{* \mathbf{0}}-2 i C^{\prime}\left[\boldsymbol{\sigma} \times \overline{\mathbf{D}}^{* \mathbf{0}}\right]\right\} .
\end{aligned}
$$

This analysis shows that the unflavored pentaquark with the spin-parity $J^{P}=3 / 2^{-}$is mainly decaying either to $J / \psi p$ final state, in which it was observed due to our assignment, or to $\Lambda_{c}^{+} \bar{D}^{* 0}$ state. 
If instead we assign $q=s$, but keeping the light diquark $d_{0}$ as above, we get two states with spins $S_{h d}=1$ from table 11 and the corresponding operators are as follows:

$$
\begin{aligned}
\Psi_{1}^{H(1 / 2)} & =\frac{i}{\sqrt{2}} \Lambda\left\{a_{s}^{\prime} \eta_{c}+\frac{b_{s}^{\prime}}{3}(\boldsymbol{\sigma} \mathbf{J} / \boldsymbol{\psi})\right\}, \\
\mathbf{\Psi}_{1}^{H(3 / 2)} & =\frac{i \sqrt{2}}{3} \Lambda\left\{b_{s}^{\prime} \mathbf{J} / \boldsymbol{\psi}-2 i c_{s}^{\prime}[\boldsymbol{\sigma} \times \mathbf{J} / \boldsymbol{\psi}]\right\}, \\
\Psi_{2}^{H(1 / 2)} & =-\frac{i}{\sqrt{2}} \Lambda_{c}^{+}\left\{A_{s}^{\prime} \bar{D}_{s}^{0}+\frac{B_{s}^{\prime}}{3}\left(\boldsymbol{\sigma} \overline{\mathbf{D}}_{\mathbf{s}}^{* \mathbf{0}}\right)\right\}, \\
\Psi_{2}^{H(3 / 2)} & =-\frac{i \sqrt{2}}{3} \Lambda_{c}^{+}\left\{B_{s}^{\prime} \overline{\mathbf{D}}_{\mathbf{s}}^{* \mathbf{0}}-2 i C_{s}^{\prime}\left[\boldsymbol{\sigma} \times \overline{\mathbf{D}}_{\mathbf{s}}^{* \mathbf{0}}\right]\right\} .
\end{aligned}
$$

It is easy to recognize that the main decays in which a singly-strange $J^{P}=3 / 2^{-}$pentaquark should be searched, are the $J / \psi \Lambda$ and $\Lambda_{c}^{+} \bar{D}_{s}^{* 0}$ final states. Analysis of the other pentaquark states can be done along similar lines.

From the foregoings in this section, it is evident that the color representations and color reconnections, on one hand, and localization of the diquarks and quarks, on the other, are essential to disentangle the dynamics (production and decays) of multiquark hadrons. We have worked out some illustrative cases here, in the color-antitriplet diquark representation, imposing heavy-quark symmetry constraints in the decays of $b$-baryons.

We end this section with a remark about the composition, localisation, and bindings of the various building blocks of a pentaquark in our model. In the compact diquark picture which we follow, the quarks in a diquark are bound and not free, and they have a hadronic size, which could be as large as several tenths of a Fermi. This applies to both the light $\left[q^{\prime} q^{\prime \prime}\right]$-diquark as well as the heavy-light $[q c]$-diquark. In this respect, the compact pentaquark model differs from the hadrocharmonium model [55], in which the light degrees of freedom are considered as a cloud. We implicitly assume that the diquark sizes are smaller than that of the triquark, which in turn is smaller than the pentaquark size. So, the compact pentaquark model is essentially a three-scale problem. The light diquark is localized at some distance from the doubly-heavy triquark and an overlap of their wave functions can be described within the one-gluon-exchange approach. The pentaquark stabilization is essentially determined by the triquark stabilization, which is accomplished by assuming that there is a barrier/bound-state-effect which reduces the probability of the $\bar{c}$ and the charmed quark in the heavy $[q c]$-diquark to annihilate each other. This barrier is essential, as otherwise the $c$ - and $\bar{c}$-quarks will immediately annihilate. The triquark topology is not too different than that of a tetraquark consisting of $[q c]$ - and $\left[\bar{q}^{\prime} \bar{c}\right]$-diquarks, where the tetraquark is stabilized due to a barrier between the diquark and the anti-diquark shells $[48,49]$.

As a final remark, we note that in the diquark models light diquarks $\left[q q^{\prime}\right]$ are ubiquitous. They are present in baryons, such as $\Lambda_{b}$, and the hidden-charm pentaquarks, discussed in detail in our paper, but also in the excited baryons, such as $\Omega_{c}^{*}=c\{s s\}$ [59], and in doubly-heavy tetraquarks, such as $\bar{b} \bar{b}\left[q q^{\prime}\right]$, discussed in a number of papers on stable tetraquarks [41-45]. How their hadronic sizes impact on properties of a given multiquark state depends on an underlying dynamical model. In the constituent diquark model, which 
we use, the size of the light diquark influences various parameters of the effective Hamiltonian. We have ignored the differences which emerge from intrinsically different embeddings of the diquarks in the baryons, tetraquarks and pentaquarks. This is an approximation, as the hadrons in question are not expected to have a universal hadronic size, and it limits the precision of the pentaquark masses presented here. A detailed dynamical scheme for pentaquarks remains to be developed - an issue which we hope to address in the future.

\section{Conclusions}

We have presented the mass spectrum of the hidden-charm pentaquark states $\left(c \bar{c} q q^{\prime} q^{\prime \prime}\right)$, where $q, q^{\prime}$, and $q^{\prime \prime}$ are light $u$-, $d,-$ and $s$-quarks, using isospin symmetry for the $u$ - and $d$-quarks. In doing this, we have used an effective Hamiltonian, based on a doubly-heavy triquark - light diquark picture, shown in figure 1. Apart from the constituent diquark and quark masses, the Hamiltonian incorporates dominant spin-spin, spin-orbit, orbital and tensor interactions. As the first step, we present analytical expressions for the masses of the states having well-defined quantum numbers, making explicit the transformations needed in the relations among various angular momentum basis vectors. This formalism is the main theoretical contribution of our paper.

For the numerical estimates of the pentaquark masses, we use Model II by Maiani et al. [56], developed to calculate the $X, Y$, and $Z$ tetraquark mass spectrum in the compact diquark model, in which only the dominant spin-spin couplings are taken into account. Since not all parameters in the effective Hamiltonian can be uniquely determined, as there are currently only three pentaquark states observed so far, and also their spinparity assignments are not known, we use the results of the known heavy tetraquarks and heavy baryons to fix them. Due to the paucity of data on pentaquarks, and the inference on the model parameters from other systems, it is difficult to be quantitatively precise. A remark about the accuracy on our mass estimates due to the neglect of the diquark size effects is in order here. While we have neglected this, it has been estimated recently for the double-bottom hadrons (baryons and tetraquarks) by taking into account gluon exchanges between the spectator quarks and the two heavy quarks in the diquark. Using the non-relativistic potential model to estimate the matrix element of the resulting dominant operator leads to a contribution $\Delta M_{\Xi_{b b q}} \simeq 30 \mathrm{MeV}$, with a similar contribution to the mass shift of the corresponding tetraquark [70]. The corresponding effect for the hidden-charm tetraquarks and pentaquarks is not yet at hand, but is presumably of a similar order of magnitude. Thus, there is an intrinsic diquark-size dependent uncertainty in our mass estimates, which we hope can be estimated in an improved dynamical diquark framework. In view of this, the masses of the pentaquarks in the compact diquark approach presented here, while being in the right ball-park of the current experimental measurements, require an improved theoretical framework and more data to be precise.

The resulting pentaquark spectrum is very rich, as the states have both spin-0 and spin-1 light diquarks. Since the current experimental effort is dominated by searches in $\Lambda_{b^{-}}$ decays, we impose heavy quark symmetry, in which the light-diquark spin in $\Lambda_{b}$-baryon is a good quantum number. This, in turn, implies that only pentaquarks with the spin-0 light 
diquarks are anticipated in $\Lambda_{b}$-decays. This reduces the spectrum of the observable states greatly, yet there are more states present in the spectrum than have been observed, and hence we are not able to assign the $J^{P}$ quantum numbers to the observed states uniquely. We have argued, based on theoretical consistency, why some spin-parity assignments for the new pentaquarks can be ruled out in the compact diquark model. Yet, by virtue of the fact that both $S$ - and $P$-state pentaquarks are anticipated in such models, predicting both positive- and negative-parity states, the spectrum provides a generic discrimination between the compact pentaquarks and the ones foreseen in hadron molecules, in which the observed pentaquarks have all the negative parity. Thus, it is crucial to determine the $J^{P}$ quantum numbers of the observed pentaquarks. Likewise, the remaining states in the pentaquark spectrum, whose masses we have presented, should be searched for. We find that some of them may lie in mass below their strong thresholds, defined by $J / \psi p$ (or $\eta_{c} p$ ). Hence, they will decay via the $c \bar{c}$-pair annihilation into light hadrons and into a lepton pair. Thus, the mode $p \mu^{+} \mu^{-}$may reveal the presence of these additional pentaquark states, and we urge the LHCb collaboration to search for them.

The hidden-charm pentaquarks having a strange quark in their valence composition are at present an experimentally virgin territory. While they can be produced also promptly, like their non-strange counterparts, only search strategies based on weak decays of the $b$-baryons with a strange quark are expected to bear fruits. The strange $b$-baryons which decay weakly are $\Xi_{b}^{0}(b u s), \Xi_{b}^{-}(b d s)$, and $\Omega_{b}^{-}(b s s)$. Of these, the decays of $\Omega_{b}^{-}(b s s)$ will produce pentaquarks with a spin-1 light diquark, in accordance with the heavy quark symmetry, thus they allow to investigate the $\mathrm{SU}(3)_{F}$-decuplet pentaquark sector. However, the production of such $b$-baryons is suppressed due to the excitation of the $s \bar{s}$-pair from the vacuum (for producing $\Xi_{b}^{0}$ and $\Xi_{b}^{-}$in a $b$-quark initiated jet) and twice suppressed (for the $\Omega_{b}^{-}$-baryon). As an example, the product branching ratio $\mathcal{B}\left(\Omega_{b}^{-} \rightarrow J / \psi \Omega^{-}\right) \mathcal{B}(b \rightarrow$ $\left.\Omega_{b}^{-}\right)=\left(2.9_{-0.8}^{+1.1}\right) \times 10^{-6}[3]$. This, compared with the product branching ratio $\mathcal{B}\left(\Lambda_{b}^{0} \rightarrow\right.$ $\left.J / \psi \Lambda^{0}\right) \mathcal{B}\left(b \rightarrow \Lambda_{b}^{0}\right)=(5.8 \pm 0.8) \times 10^{-5}$, is about 20 times less probable. Thus, the discovery of hidden-charm strange pentaquarks requires a lot more data, but they remain a firm prediction, as in the compact diquark model complete $\mathrm{SU}(3)_{F}$-multiplets are anticipated. Hence, their non-observation in experiments would seriously undermine such models. In the foreseeable future, experiments at the LHC, in particular LHCb, remain our best hope for their discovery. In the long-term, the entire hidden-charm pentaquark sector can be studied at a Tera- $Z$ factory.

\section{Acknowledgments}

We would like to thank Vladimir Galkin, Marek Karliner, Luciano Maiani, Antonello Polosa, Tomasz Skwarnicki, and Sheldon Stone for helpful discussions. A. A. and A.P. acknowledge support and warm hospitality of the Theoretical Physics Division at IHEP (Beijing, China) where an initial part of this research was performed, and Wei Wang for the hospitality at the Laboratory of Particle Physics of the Shanghai Jiao-Tong University, where it was completed. A. P. thanks the Theory Group at DESY for their kind hospitality, where the major part of this work was done. A.P. acknowledges financial support 
by the German Academic Exchange Service (DAAD), by the Russian Foundation for Basic Research and National Natural Science Foundation of China for the research project No. 19-52-53041, and by the "YSU Initiative Scientific Research Activity" (Project No. AAAA-A16-116070610023-3).

\section{A Corrections due to spin-spin interactions between the constituents of doubly-heavy triquark and light diquark}

The matrix elements of the operators in eq. (3.4) have in general six different coupling strengths. For the pentaquarks, which contain the light $u$ - and $d$-quarks only, isospin symmetry among the light quarks can be used to reduce the number of couplings: $\left(\tilde{\mathcal{K}}_{c q^{\prime}}\right) \overline{3}=$ $\left(\tilde{\mathcal{K}}_{c q^{\prime \prime}}\right)_{\overline{3}}=\left(\tilde{\mathcal{K}}_{c q}\right)_{\overline{3}},\left(\tilde{\mathcal{K}}_{q q^{\prime}}\right)_{\overline{3}}=\left(\tilde{\mathcal{K}}_{q q^{\prime \prime}}\right)_{\overline{3}}=\left(\tilde{\mathcal{K}}_{q q}\right)_{\overline{3}}$, and $\tilde{\mathcal{K}}_{\bar{c} q^{\prime}}=\tilde{\mathcal{K}}_{\bar{c} q^{\prime \prime}}=\tilde{\mathcal{K}}_{\bar{c} q}$. So, the expression for $H_{S S}^{t-l d}$ in the effective Hamiltonian responsible for the spin-spin interactions between the (anti)quarks in the diquark and triquark, given in eq. (3.4), is greatly simplified:

$$
H_{S S}^{t-l d}=2 \tilde{\mathcal{K}}_{\bar{c} q}\left(\mathbf{S}_{\bar{c}} \cdot \mathbf{S}_{l d}\right)+2\left(\tilde{\mathcal{K}}_{c q}\right) \overline{3}\left(\mathbf{S}_{c} \cdot \mathbf{S}_{l d}\right)+2\left(\tilde{\mathcal{K}}_{q q}\right) \overline{3}\left(\mathbf{S}_{q} \cdot \mathbf{S}_{l d}\right),
$$

where the definition of the light-diquark spin operator, $\mathbf{S}_{l d}=\mathbf{S}_{q^{\prime}}+\mathbf{S}_{q^{\prime \prime}}$, is used. We start by calculating the matrix elements of the spin-spin operators from the first term, $2 \tilde{\mathcal{K}}_{\bar{c} q}\left(\mathbf{S}_{\bar{c}} \cdot \mathbf{S}_{l d}\right)$, which contains the spins of the charm antiquark and light diquark. Again, it is convenient to transform the vector basis. To do so, we denote the pentaquark state $\left|S_{h d}, S_{t}, L_{t} ; S_{l d}, L_{l d} ; S, L\right\rangle_{J}$ as $\left|S_{l d},\left(S_{\bar{c}}, S_{h d}\right)_{S_{t}} ; S\right\rangle$ and apply the transformation (4.4):

$$
\begin{aligned}
& \left|S_{l d},\left(S_{\bar{c}}, S_{h d}\right)_{S_{t}} ; S\right\rangle=\sum_{\tilde{S}}(-1)^{S_{l d}+S_{\bar{c}}+S_{h d}+S} \sqrt{(2 \tilde{S}+1)\left(2 S_{t}+1\right)} \\
& \times\left\{\begin{array}{ccc}
S_{l d} & S_{\bar{c}} & \tilde{S} \\
S_{h d} & S & S_{t}
\end{array}\right\}\left|\left(S_{l d}, S_{\bar{c}}\right)_{\tilde{S}}, S_{h d} ; S\right\rangle .
\end{aligned}
$$

The required matrix elements can be written in the form:

$$
\begin{aligned}
& { }_{J}\left\langle S_{h d}, S_{t}^{\prime}, L_{t} ; S_{l d}, L_{l d} ; S, L\left|2 \tilde{\mathcal{K}}_{\bar{c} q}\left(\mathbf{S}_{\bar{c}} \cdot \mathbf{S}_{l d}\right)\right| S_{h d}, S_{t}, L_{t} ; S_{l d}, L_{l d} ; S, L\right\rangle_{J} \\
& =\tilde{\mathcal{K}}_{\bar{c} q}(-1)^{2 S+1} \sqrt{\left(2 S_{t}+1\right)\left(2 S_{t}^{\prime}+1\right)} \sum_{\tilde{S}}(2 \tilde{S}+1) \\
& \quad \times\left[\tilde{S}(\tilde{S}+1)-S_{l d}\left(S_{l d}+1\right)-\frac{3}{4}\right]\left\{\begin{array}{ccc}
S_{l d} & 1 / 2 & \tilde{S} \\
S_{h d} & S & S_{t}
\end{array}\right\}\left\{\begin{array}{ccc}
S_{l d} & 1 / 2 & \tilde{S} \\
S_{h d} & S & S_{t}^{\prime}
\end{array}\right\},
\end{aligned}
$$

where the charm-antiquark spin, $S_{\bar{c}}=1 / 2$, is substituted.

We calculate now the matrix elements of the last two operators in eq. (A.1). Let us start from the operator $2\left(\tilde{\mathcal{K}}_{c q}\right) \overline{3}\left(\mathbf{S}_{c} \cdot \mathbf{S}_{l d}\right)$ which works on the wave functions of the charm quark in the heavy diquark and light diquark. To find it, we need to couple the heavy and light diquark first and then couple the light diquark with the charm quark - the constituent of heavy-light diquark. This requires a double transformation of the original pentaquark vector state $\left|S_{h d}, S_{t}, L_{t} ; S_{l d}, L_{l d} ; S, L\right\rangle_{J}$, which we denote as $\left|S_{l d},\left(S_{h d}, S_{\bar{c}}\right)_{S_{t}} ; S\right\rangle$, to the vector 
state $\left|\left(S_{l d}, S_{h d}\right)_{S_{1}}, S_{\bar{c}} ; S\right\rangle$; in the second transformation these basis states, for simplicity called as $\left|S_{l d},\left(S_{c}, S_{q}\right)_{S_{h d}} ; S_{1}\right\rangle$, are changed to the required ones, $\left|\left(S_{l d}, S_{c}\right)_{S_{2}}, S_{q} ; S_{1}\right\rangle$. Specifically:

$$
\begin{aligned}
\mid S_{l d}, & \left(S_{h d}, S_{\left.\bar{c})_{S_{t}} ; S\right\rangle=} \sum_{S_{1}}(-1)^{S_{l d}+S_{h d}+S_{\bar{c}}+S} \sqrt{\left(2 S_{1}+1\right)\left(2 S_{t}+1\right)}\right. \\
& \times\left\{\begin{array}{ccc}
S_{l d} & S_{h d} & S_{1} \\
S_{\bar{c}} & S & S_{t}
\end{array}\right\}\left|\left(S_{l d}, S_{h d}\right)_{S_{1}}, S_{\bar{c}} ; S\right\rangle \\
= & \sum_{S_{1}}(-1)^{S_{l d}+S_{h d}+S_{\bar{c}}+S} \sqrt{\left(2 S_{1}+1\right)\left(2 S_{t}+1\right)}\left\{\begin{array}{ccc}
S_{l d} & S_{h d} S_{1} \\
S_{\bar{c}} & S & S_{t}
\end{array}\right\}\left|S_{l d},\left(S_{c}, S_{q}\right)_{S_{h d}} ; S_{1}\right\rangle \\
= & \sum_{S_{1}, S_{2}}(-1)^{2 S_{l d}+S_{h d}+S_{\bar{c}}+S+S_{c}+S_{q}+S_{1}} \sqrt{\left(2 S_{1}+1\right)\left(2 S_{t}+1\right)\left(2 S_{h d}+1\right)\left(2 S_{2}+1\right)} \\
& \times\left\{\begin{array}{ccc}
S_{l d} & S_{h d} & S_{1} \\
S_{\bar{c}} & S & S_{t}
\end{array}\right\}\left\{\begin{array}{ccc}
S_{l d} & S_{c} & S_{2} \\
S_{q} & S_{1} & S_{h d}
\end{array}\right\}\left|\left(S_{l d}, S_{c}\right)_{S_{2}}, S_{q} ; S_{1}\right\rangle .
\end{aligned}
$$

The matrix elements of interest are as follows:

$$
\begin{aligned}
{ }_{J}\left\langle S_{h d}^{\prime}, S_{t}^{\prime}, L_{t} ; S_{l d}, L_{l d} ; S, L\left|2\left(\tilde{\mathcal{K}}_{c q}\right) \overline{3}\left(\mathbf{S}_{c} \cdot \mathbf{S}_{l d}\right)\right| S_{h d}, S_{t}, L_{t} ; S_{l d}, L_{l d} ; S, L\right\rangle_{J} \\
=\left(\tilde{\mathcal{K}}_{c q}\right) \overline{3}(-1)^{S_{h d}+S_{h d}^{\prime}+2 S+1} \sqrt{\left(2 S_{h d}+1\right)\left(2 S_{h d}^{\prime}+1\right)\left(2 S_{t}+1\right)\left(2 S_{t}^{\prime}+1\right)} \\
\quad \times \sum_{S_{1}, S_{2}}\left(2 S_{1}+1\right)\left(2 S_{2}+1\right)\left[S_{2}\left(S_{2}+1\right)-S_{l d}\left(S_{l d}+1\right)-\frac{3}{4}\right] \\
\quad \times\left\{\begin{array}{ccc}
S_{l d} & S_{h d} & S_{1} \\
1 / 2 & S & S_{t}
\end{array}\right\}\left\{\begin{array}{ccc}
S_{l d} & S_{h d}^{\prime} S_{1} \\
1 / 2 & S & S_{t}^{\prime}
\end{array}\right\}\left\{\begin{array}{ccc}
S_{l d} & 1 / 2 & S_{2} \\
1 / 2 & S_{1} & S_{h d}
\end{array}\right\}\left\{\begin{array}{ccc}
S_{l d} & 1 / 2 & S_{2} \\
1 / 2 & S_{1} & S_{h d}^{\prime}
\end{array}\right\},
\end{aligned}
$$

where the quark and antiquark spins, $S_{c}=S_{q}=S_{\bar{c}}=1 / 2$, are substituted. Note that the matrix elements of the last operator in (A.1) $2\left(\tilde{\mathcal{K}}_{q q}\right)_{\overline{3}}\left(\mathbf{S}_{q} \cdot \mathbf{S}_{l d}\right)$ differ from the above matrix elements by the replacement $\left(\tilde{\mathcal{K}}_{c q}\right)_{\overline{3}} \rightarrow\left(\tilde{\mathcal{K}}_{q q}\right)_{\overline{3}}$ and the interchange $S_{c}=1 / 2 \leftrightarrow S_{q}=1 / 2$. It means that, up to the coupling strengths, they coincide. So, the contribution of these two operators enters the matrix elements with the factor $\left(\tilde{\mathcal{K}}_{c q}\right) \overline{3}+\left(\tilde{\mathcal{K}}_{q q}\right) \overline{3}$.

We continue by considering the spin-spin interaction between the strange hidden-charm triquark and unflavored (i.e., having $u$ - and $d$-quarks) light diquark. In this case, the expression given in eq. (3.4) of the effective Hamiltonian is again simplified in the isospinsymmetry limit:

$$
H_{S S}^{t-l d}=2 \tilde{\mathcal{K}}_{\bar{c} q}\left(\mathbf{S}_{\bar{c}} \cdot \mathbf{S}_{l d}\right)+2\left(\tilde{\mathcal{K}}_{c q}\right) \overline{3}\left(\mathbf{S}_{c} \cdot \mathbf{S}_{l d}\right)+2\left(\tilde{\mathcal{K}}_{s q}\right) \overline{3}\left(\mathbf{S}_{s} \cdot \mathbf{S}_{l d}\right)
$$

where $\mathbf{S}_{s}$ and $\mathbf{S}_{l d}=\mathbf{S}_{q^{\prime}}+\mathbf{S}_{q^{\prime \prime}}$ are the spin operators of the $s$-quark in the triquark and the light-diquark, respectively. Comparing (A.6) with (A.1), it is easy to recognize that they differ by the factor entering the last terms, $\left(\tilde{\mathcal{K}}_{s q}\right)_{\overline{3}}$ and $\left.\left(\tilde{\mathcal{K}}_{q q}\right)\right)_{3}$. So, the matrix elements (A.3) and (A.5) remain unchanged, but to get the contribution of the last operator in (A.6) one should simply change the coupling: $\left(\tilde{\mathcal{K}}_{q q}\right)_{\overline{3}} \rightarrow\left(\tilde{\mathcal{K}}_{s q}\right)_{\overline{3}}$. Taking into account the statement in the paragraph above, the second and third terms in (A.6) are equal (modulo couplings) and contribute with the coefficient $\left(\tilde{\mathcal{K}}_{c q}\right)_{\overline{3}}+\left(\tilde{\mathcal{K}}_{s q}\right)_{\overline{3}}$ in the mass formulae. 
The same simple form of the spin-spin interaction is also hold for the hidden-charm pentaquark with the strangeness $S=-2$ which consists of the unflavored doubly-heavy triquark and light diquark containing two $s$-quarks:

$$
H_{S S}^{t-l d}=2 \tilde{\mathcal{K}}_{\bar{c} s}\left(\mathbf{S}_{\bar{c}} \cdot \mathbf{S}_{l d}\right)+2\left(\tilde{\mathcal{K}}_{c s}\right)_{\overline{3}}\left(\mathbf{S}_{c} \cdot \mathbf{S}_{l d}\right)+2\left(\tilde{\mathcal{K}}_{s q}\right)_{\overline{3}}\left(\mathbf{S}_{q} \cdot \mathbf{S}_{l d}\right) .
$$

So, the matrix elements (A.3) and (A.5), up to spin-spin couplings, remain unchanged and their contributions to the mass formulae are accompanied by the couplings $\tilde{\mathcal{K}}_{\bar{c} s}$ and $\left(\tilde{\mathcal{K}}_{c s}\right)_{\overline{3}}+\left(\tilde{\mathcal{K}}_{s q}\right) \overline{3}$, respectively.

Similar simple effective Hamiltonian for the spin-spin interactions is present in the hidden-charm pentaquarks with the strangeness $S=-3$, which contains three $s$-quarks:

$$
H_{S S}^{t-l d}=2 \tilde{\mathcal{K}}_{\bar{c} s}\left(\mathbf{S}_{\bar{c}} \cdot \mathbf{S}_{l d}\right)+2\left(\tilde{\mathcal{K}}_{c s}\right)_{\overline{3}}\left(\mathbf{S}_{c} \cdot \mathbf{S}_{l d}\right)+2\left(\tilde{\mathcal{K}}_{s s}\right)_{\overline{3}}\left(\mathbf{S}_{s} \cdot \mathbf{S}_{l d}\right) .
$$

The matrix elements (A.3) and (A.5) can be safely used in this case also and their contributions are entering the mass formulae with the couplings $\tilde{\mathcal{K}}_{\bar{c} s}$ and $\left(\tilde{\mathcal{K}}_{c s}\right)_{\overline{3}}+\left(\tilde{\mathcal{K}}_{s s}\right) \overline{3}$, respectively.

The next flavor state which corresponds to the hidden-charm strange pentaquark consists of the the unflavored doubly-heavy triquark and strange light diquark. The corresponding spin-spin effective Hamiltonian (3.4) does not simplified and all the six terms, each of which is accompanied by its own coupling, should be considered separately:

$$
\begin{aligned}
H_{S S}^{t-l d}= & 2 \tilde{\mathcal{K}}_{\bar{c} q}\left(\mathbf{S}_{\bar{c}} \cdot \mathbf{S}_{q^{\prime}}\right)+2\left(\tilde{\mathcal{K}}_{c q}\right)_{\overline{3}}\left(\mathbf{S}_{c} \cdot \mathbf{S}_{q^{\prime}}\right)+2\left(\tilde{\mathcal{K}}_{q q}\right) \overline{3}\left(\mathbf{S}_{q} \cdot \mathbf{S}_{q^{\prime}}\right) \\
& +2 \tilde{\mathcal{K}}_{\bar{c} s}\left(\mathbf{S}_{\bar{c}} \cdot \mathbf{S}_{s}\right)+2\left(\tilde{\mathcal{K}}_{c s}\right)_{\overline{3}}\left(\mathbf{S}_{c} \cdot \mathbf{S}_{s}\right)+2\left(\tilde{\mathcal{K}}_{s q}\right)_{\overline{3}}\left(\mathbf{S}_{q} \cdot \mathbf{S}_{s}\right)
\end{aligned}
$$

where we identify $q^{\prime \prime}=s$ and apply the isospin symmetry to the spin-spin couplings.

A calculation of these operators requires a recoupling of four angular momenta. In general, this transformation scheme is expressed through the $9 j$-symbol which is defined as follows [60]:

$$
\begin{aligned}
& \left\langle\left(j_{1}, j_{2}\right)_{j_{12}},\left(j_{3}, j_{4}\right)_{j_{34}}, j \mid\left(j_{1}, j_{3}\right)_{j_{13}},\left(j_{2}, j_{4}\right)_{j_{24}}, j\right\rangle \\
& \quad=\sqrt{\left(2 j_{12}+1\right)\left(2 j_{34}+1\right)\left(2 j_{13}+1\right)\left(2 j_{24}+1\right)}\left\{\begin{array}{ccc}
j_{1} & j_{2} & j_{12} \\
j_{3} & j_{4} & j_{34} \\
j_{13} & j_{24} & j
\end{array}\right\} .
\end{aligned}
$$

To demonstrate its necessity, let us consider the first term in (A.9). In our basis of states the charm antiquark belongs to the doubly-heavy triquark while the light $q^{\prime}$-quark is inside the light diquark. The original pentaquark vector state $\left|S_{h d}, S_{t}, L_{t} ; S_{l d}, L_{l d} ; S, L\right\rangle_{J}$, which we denote for simplicity as $\left|\left(S_{\bar{c}}, S_{h d}\right)_{S_{t}},\left(S_{q^{\prime}}, S_{s}\right)_{S_{l d}} ; S\right\rangle$, should be transformed to the new state with the recoupling momenta $\left|\left(S_{\bar{c}}, S_{q^{\prime}}\right)_{S_{\bar{c} q^{\prime}}},\left(S_{h d}, S_{s}\right)_{S_{h d, s}} ; S\right\rangle$, and the $9 j$-symbol is responsible for this transform:

$$
\begin{aligned}
\left|\left(S_{\bar{c}}, S_{h d}\right)_{S_{t}},\left(S_{q^{\prime}}, S_{s}\right)_{S_{l d}} ; S\right\rangle= & \sum_{S_{\bar{c} q^{\prime}}, S_{h d, s}} \sqrt{\left(2 S_{t}+1\right)\left(2 S_{l d}+1\right)\left(2 S_{\bar{c} q^{\prime}}+1\right)\left(2 S_{h d, s}+1\right)} \\
& \times\left\{\begin{array}{ccc}
S_{\bar{c}} & S_{h d} & S_{t} \\
S_{q^{\prime}} & S_{s} & S_{l d} \\
S_{\bar{c} q^{\prime}} & S_{h d, s} & S
\end{array}\right\}\left|\left(S_{\bar{c}}, S_{q^{\prime}}\right)_{S_{\bar{c} q^{\prime}}},\left(S_{h d}, S_{s}\right)_{S_{h d, s}} ; S\right\rangle .(A .1
\end{aligned}
$$


So, the matrix element of the operator $2 \tilde{\mathcal{K}}_{\bar{c} q}\left(\mathbf{S}_{\bar{c}} \cdot \mathbf{S}_{q^{\prime}}\right)$ sandwiched between the states with different triquark $S_{t}^{(\prime)}$ and light-diquark $S_{l d}^{(\prime)}$ vector states can be written as a double sum (here, we come back to the original notation for the vector state):

$$
\begin{aligned}
& { }_{J}\left\langle S_{h d}, S_{t}^{\prime}, L_{t} ; S_{l d}^{\prime}, L_{l d} ; S, L\left|2 \tilde{\mathcal{K}}_{\bar{c} q}\left(\mathbf{S}_{\bar{c}} \cdot \mathbf{S}_{q^{\prime}}\right)\right| S_{h d}, S_{t}, L_{t} ; S_{l d}, L_{l d} ; S, L\right\rangle_{J} \\
& =\tilde{\mathcal{K}}_{\bar{c} q} \sqrt{\left(2 S_{t}+1\right)\left(2 S_{t}^{\prime}+1\right)\left(2 S_{h d}+1\right)\left(2 S_{h d}^{\prime}+1\right)} \sum_{S_{\bar{c} q^{\prime}}, S_{h d, s}}\left(2 S_{\bar{c} q^{\prime}}+1\right)\left(2 S_{h d, s}+1\right) \\
& \quad \times\left[S_{\bar{c} q^{\prime}}\left(S_{\bar{c} q^{\prime}}+1\right)-\frac{3}{2}\right]\left\{\begin{array}{ccc}
1 / 2 & S_{h d} & S_{t}^{\prime} \\
1 / 2 & 1 / 2 & S_{l d}^{\prime} \\
S_{\bar{c} q^{\prime}} & S_{h d, s} & S
\end{array}\right\}\left\{\begin{array}{ccc}
1 / 2 & S_{h d} & S_{t} \\
1 / 2 & 1 / 2 & S_{l d} \\
S_{\bar{c} q^{\prime}} & S_{h d, s} & S
\end{array}\right\}
\end{aligned}
$$

where the spins $S_{\bar{c}}=S_{q^{\prime}}=S_{s}=1 / 2$ of quarks were subsituted. In a similar manner one can get the contribution of the operator $2 \tilde{\mathcal{K}}_{\bar{c} s}\left(\mathbf{S}_{\bar{c}} \cdot \mathbf{S}_{s}\right)$ with the result:

$$
\begin{aligned}
& { }_{J}\left\langle S_{h d}, S_{t}^{\prime}, L_{t} ; S_{l d}^{\prime}, L_{l d} ; S, L\left|2 \tilde{\mathcal{K}}_{\bar{c} s}\left(\mathbf{S}_{\bar{c}} \cdot \mathbf{S}_{s}\right)\right| S_{h d}, S_{t}, L_{t} ; S_{l d}, L_{l d} ; S, L\right\rangle_{J} \\
& =\tilde{\mathcal{K}}_{\bar{c} s} \sqrt{\left(2 S_{t}+1\right)\left(2 S_{t}^{\prime}+1\right)\left(2 S_{h d}+1\right)\left(2 S_{h d}^{\prime}+1\right)} \sum_{S_{\bar{c} s}, S_{h d, q^{\prime}}}\left(2 S_{\bar{c} s}+1\right)\left(2 S_{h d, q^{\prime}}+1\right) \\
& \quad \times\left[S_{\bar{c} s}\left(S_{\bar{c} s}+1\right)-\frac{3}{2}\right]\left\{\begin{array}{ccc}
1 / 2 & S_{h d} & S_{t}^{\prime} \\
1 / 2 & 1 / 2 & S_{l d}^{\prime} \\
S_{\bar{c} s} & S_{h d, q^{\prime}} & S
\end{array}\right\}\left\{\begin{array}{ccc}
1 / 2 & S_{h d} & S_{t} \\
1 / 2 & 1 / 2 & S_{l d} \\
S_{\bar{c} s} & S_{h d, q^{\prime}} & S
\end{array}\right\} .
\end{aligned}
$$

Up to the coupling, $\tilde{\mathcal{K}}_{\bar{c} s}$, this equation coincides with (A.12), so their common contribution contains the factor $\tilde{\mathcal{K}}_{\bar{c} q}+\tilde{\mathcal{K}}_{\bar{c} s}$.

With the other four operators the situation is a little bit more complicated as spin-spin interactions relate quarks from different diquarks, moreover, the spin of the heavy diquark is coupled with the spin of the charm antiquark inside the triquark system. So, at the first step one needs to decouple the heavy diquark from the charm antiquark and couple it with the light diquark which generates the tetraquark system. If we denote the vector state as $\left|S_{l d},\left(S_{h d}, S_{\bar{c}}\right)_{S_{t}} ; S\right\rangle$, we need to recouple in a way to get the new basis $\left|\left(S_{l d}, S_{h d},\right)_{S_{T}} S_{\bar{c}} ; S\right\rangle$ which is possible to do with the help of the transformation (4.4):

$$
\begin{aligned}
\left|S_{l d},\left(S_{h d}, S_{\bar{c}}\right)_{S t} ; S\right\rangle= & \sum_{S_{T}}(-1)^{S_{l d}+S_{h d}+S_{\bar{c}}+S} \sqrt{\left(2 S_{T}+1\right)\left(2 S_{t}+1\right)} \\
& \times\left\{\begin{array}{ccc}
S_{l d} & S_{h d} S_{T} \\
S_{\bar{c}} & S & S_{t}
\end{array}\right\}\left|\left(S_{l d}, S_{h d}\right)_{S_{T}}, S_{\bar{c}} ; S\right\rangle .
\end{aligned}
$$

At the second step, it is necessary to recouple quark spins inside the tetraquark. So, for this transformation the $9 j$-symbol (A.10) is required. To do this, the introduced pentaquark vector state $\left|\left(S_{l d}, S_{h d}\right)_{S_{T}}, S_{\bar{c}} ; S\right\rangle$ is more convenient to rewrite in the form 
$\left|\left(S_{c}, S_{q}\right)_{S_{h d}},\left(S_{q^{\prime}}, S_{s}\right)_{S_{l d}} ; S_{T}\right\rangle$ and then perform the transformations:

$$
\begin{aligned}
\left|\left(S_{c}, S_{q}\right)_{S_{h d}},\left(S_{q^{\prime}}, S_{s}\right)_{S_{l d}} ; S_{T}\right\rangle= & \sum_{S_{c q^{\prime}}, S_{s q}} \sqrt{\left(2 S_{h d}+1\right)\left(2 S_{l d}+1\right)\left(2 S_{c q^{\prime}}+1\right)\left(2 S_{s q}+1\right)} \\
& \times\left\{\begin{array}{ccc}
S_{c} & S_{q} & S_{h d} \\
S_{q^{\prime}} & S_{s} & S_{l d} \\
S_{c q^{\prime}} & S_{s q} & S_{T}
\end{array}\right\}\left|\left(S_{c}, S_{q^{\prime}}\right)_{S_{c q^{\prime}}},\left(S_{q}, S_{s}\right)_{S_{s q}} ; S_{T}\right\rangle .
\end{aligned}
$$

Now, we can sandwich the operators $2\left(\tilde{\mathcal{K}}_{c q}\right) \overline{3}\left(\mathbf{S}_{c} \cdot \mathbf{S}_{q^{\prime}}\right)$ and $2\left(\tilde{\mathcal{K}}_{s q}\right)_{\overline{3}}\left(\mathbf{S}_{s} \cdot \mathbf{S}_{q}\right)$ by the vector states with varying spins of the heavy, $S_{h d}$, and light, $S_{l d}$, diquarks as well as of the doublyheavy triquark, $S_{t}$. The resulting matrix element of these two operators is the triple sum:

$$
\begin{aligned}
& J\left\langle S_{h d}^{\prime}, S_{t}^{\prime}, L_{t} ; S_{l d}^{\prime}, L_{l d} ; S, L\left|2\left(\tilde{\mathcal{K}}_{c q}\right) \overline{3}\left(\mathbf{S}_{c} \cdot \mathbf{S}_{q^{\prime}}\right)+2\left(\tilde{\mathcal{K}}_{s q}\right) \overline{3}\left(\mathbf{S}_{s} \cdot \mathbf{S}_{q}\right)\right| S_{h d}, S_{t}, L_{t} ; S_{l d}, L_{l d} ; S, L\right\rangle_{J} \\
&= {\left[\left(\tilde{\mathcal{K}}_{c q}\right)_{\overline{3}}+\left(\tilde{\mathcal{K}}_{s q}\right)_{\overline{3}}\right] \sqrt{\left(2 S_{t}+1\right)\left(2 S_{t}^{\prime}+1\right)\left(2 S_{h d}+1\right)\left(2 S_{h d}^{\prime}+1\right)\left(2 S_{l d}+1\right)\left(2 S_{l d}^{\prime}+1\right)} } \\
& \times(-1)^{S_{h d}+S_{h d}^{\prime}+S_{l d}+S_{l d}^{\prime}+2 S+1} \sum_{S_{T}, S_{c q^{\prime}}, S_{s q}}\left(2 S_{T}+1\right)\left(2 S_{c q^{\prime}}+1\right)\left(2 S_{s q}+1\right)\left[S_{c q^{\prime}}\left(S_{c q^{\prime}}+1\right)-\frac{3}{2}\right] \\
& \times\left\{\begin{array}{ccc}
S_{l d}^{\prime} & S_{h d}^{\prime} & S_{T} \\
1 / 2 & S & S_{t}^{\prime}
\end{array}\right\}\left\{\begin{array}{ccc}
S_{l d} & S_{h d} & S_{T} \\
1 / 2 & S & S_{t}
\end{array}\right\}\left\{\begin{array}{lll}
1 / 2 & 1 / 2 & S_{h d}^{\prime} \\
1 / 2 & 1 / 2 & S_{l d}^{\prime} \\
S_{c q^{\prime}} & S_{s q} & S_{T}
\end{array}\right\}\left\{\begin{array}{lll}
1 / 2 & 1 / 2 & S_{h d} \\
1 / 2 & 1 / 2 & S_{l d} \\
S_{c q^{\prime}} & S_{s q} & S_{T}
\end{array}\right\} .
\end{aligned}
$$

The matrix elements of the rest two operators $2\left(\tilde{\mathcal{K}}_{c s}\right) \overline{3}\left(\mathbf{S}_{c} \cdot \mathbf{S}_{s}\right)$ and $2\left(\tilde{\mathcal{K}}_{q q}\right) \overline{3}\left(\mathbf{S}_{q} \cdot \mathbf{S}_{q^{\prime}}\right)$ are calculated similarly with the following result:

$$
\begin{aligned}
&{ }_{J}\left\langle S_{h d}^{\prime}, S_{t}^{\prime}, L_{t} ; S_{l d}^{\prime}, L_{l d} ; S, L\left|2\left(\tilde{\mathcal{K}}_{c s}\right)_{\overline{3}}\left(\mathbf{S}_{c} \cdot \mathbf{S}_{s}\right)+2\left(\tilde{\mathcal{K}}_{q q}\right)_{\overline{3}}\left(\mathbf{S}_{q} \cdot \mathbf{S}_{q^{\prime}}\right)\right| S_{h d}, S_{t}, L_{t} ; S_{l d}, L_{l d} ; S, L\right\rangle_{J} \\
&= {\left[\left(\tilde{\mathcal{K}}_{c s}\right)_{\overline{3}}+\left(\tilde{\mathcal{K}}_{q q}\right)_{\overline{3}}\right] \sqrt{\left(2 S_{t}+1\right)\left(2 S_{t}^{\prime}+1\right)\left(2 S_{h d}+1\right)\left(2 S_{h d}^{\prime}+1\right)\left(2 S_{l d}+1\right)\left(2 S_{l d}^{\prime}+1\right)} } \\
& \times(-1)^{S_{h d}+S_{h d}^{\prime}+S_{l d}+S_{l d}^{\prime}+2 S+1} \sum_{S_{T}, S_{c s}, S_{q q^{\prime}}}\left(2 S_{T}+1\right)\left(2 S_{c s}+1\right)\left(2 S_{q q^{\prime}}+1\right)\left[S_{c s}\left(S_{c s}+1\right)-\frac{3}{2}\right] \\
& \times\left\{\begin{array}{ccc}
S_{l d}^{\prime} & S_{h d}^{\prime} & S_{T} \\
1 / 2 & S & S_{t}^{\prime}
\end{array}\right\}\left\{\begin{array}{ccc}
S_{l d} & S_{h d} & S_{T} \\
1 / 2 & S & S_{t}
\end{array}\right\}\left\{\begin{array}{lll}
1 / 2 & 1 / 2 & S_{h d}^{\prime} \\
1 / 2 & 1 / 2 & S_{l d}^{\prime} \\
S_{c s} & S_{q q^{\prime}} & S_{T}
\end{array}\right\}\left\{\begin{array}{lll}
1 / 2 & 1 / 2 & S_{h d} \\
1 / 2 & 1 / 2 & S_{l d} \\
S_{c s} & S_{q q^{\prime}} & S_{T}
\end{array}\right\} .
\end{aligned}
$$

In general, eqs. (A.16) and (A.17) are the matrices which mix all the states having the same total spin, $S$, and the same parity.

The last flavor state which corresponds to the hidden-charm strange pentaquark with the strangeness $S=-2$ consists of the the strange doubly-heavy triquark and strange light diquark. The corresponding spin-spin effective Hamiltonian (3.4) does not get simplified in this case also, and all the six terms, each of which is accompanied by its own coupling, should be considered separately:

$$
\begin{aligned}
H_{S S}^{t-l d}= & 2 \tilde{\mathcal{K}}_{\bar{c} q}\left(\mathbf{S}_{\bar{c}} \cdot \mathbf{S}_{q}\right)+2\left(\tilde{\mathcal{K}}_{c q}\right)_{\overline{3}}\left(\mathbf{S}_{c} \cdot \mathbf{S}_{q}\right)+2\left(\tilde{\mathcal{K}}_{s q}\right)_{\overline{3}}\left(\mathbf{S}_{s} \cdot \mathbf{S}_{q}\right) \\
& +2 \tilde{\mathcal{K}}_{\bar{c} s}\left(\mathbf{S}_{\bar{c}} \cdot \mathbf{S}_{s^{\prime}}\right)+2\left(\tilde{\mathcal{K}}_{c s}\right)_{\overline{3}}\left(\mathbf{S}_{c} \cdot \mathbf{S}_{s^{\prime}}\right)+2\left(\tilde{\mathcal{K}}_{s s}\right) \overline{3}\left(\mathbf{S}_{s} \cdot \mathbf{S}_{s^{\prime}}\right)
\end{aligned}
$$


where we make the substitution $q=s, q^{\prime}=q$, and $q^{\prime \prime}=s^{\prime}$. The above consideration holds also here, and one can use the expressions (A.13), (A.16), and (A.17) with the obvious replacements $\left(\tilde{\mathcal{K}}_{s q}\right)_{\overline{3}} \rightarrow\left(\tilde{\mathcal{K}}_{s s}\right)_{\overline{3}}$ in $(\mathrm{A} .16)$ and $\left(\tilde{\mathcal{K}}_{q q}\right)_{\overline{3}} \rightarrow\left(\tilde{\mathcal{K}}_{s q}\right)_{\overline{3}}$ in $(\mathrm{A} .17)$.

The contributions discussed in this appendix are relevant for the Model I by Maiani et al. [35], where such (subdominant) spin-spin interactions are included in the effective Hamiltonian. We presented the formalism here. They are neglected in the numerical analysis given in this paper, as we use the Model II [56] for our mass estimates.

\section{B Mass derivations for the $\boldsymbol{P}$-wave pentaquark states}

\section{B.1 The states with $J^{P}=3 / 2^{+}$and triquark spin $S_{t}=3 / 2$}

The mass matrix of the states with the spin-parity $J^{P}=3 / 2^{+}$and triquark spin $S_{t}=1 / 2$ is as follows:

$$
\begin{aligned}
\tilde{M}_{J=3 / 2}^{P 1}= & M_{0}+\frac{1}{2}\left(\mathcal{K}_{c q}\right)_{\overline{3}}+\frac{1}{2}\left(\mathcal{K}_{q^{\prime} q^{\prime \prime}}\right)_{\overline{3}}+\frac{1}{2}\left(\mathcal{K}_{\bar{c} q}+\mathcal{K}_{\bar{c} c}\right)+B \\
& +\frac{1}{15} A_{t}\left(\begin{array}{ccc}
25 & 25 \sqrt{2} & 0 \\
25 \sqrt{2} & -22 & 9 \sqrt{6} \\
0 & 9 \sqrt{6} & -63
\end{array}\right)+\frac{1}{15} A_{l d}\left(\begin{array}{ccc}
-10 & 25 \sqrt{2} & 0 \\
25 \sqrt{2} & -8 & 9 \sqrt{6} \\
0 & 9 \sqrt{6} & -42
\end{array}\right) \\
& +b\left(\begin{array}{ccc}
-10 / 3 & \sqrt{2} / 4 & -3 \sqrt{3} / 5 \\
\sqrt{2} / 4 & -1256 / 375 & 81 \sqrt{6} / 100 \\
-3 \sqrt{3} / 5 & 81 \sqrt{6} / 100 & -372 / 125
\end{array}\right) .
\end{aligned}
$$

Before calculating the mass spectrum, we make the three matrices above to be traceless:

$$
\begin{aligned}
\tilde{M}_{J=3 / 2}^{P 1}= & M_{0}+\frac{1}{2}\left(\mathcal{K}_{c q}\right)_{\overline{3}}+\frac{1}{2}\left(\mathcal{K}_{q^{\prime} q^{\prime \prime}}\right)_{\overline{3}}+\frac{1}{2}\left(\mathcal{K}_{\bar{c} q}+\mathcal{K}_{\bar{c} c}\right)+B-\frac{4}{3}\left(A_{t}+A_{l d}\right)-\frac{3622}{1125} b \\
& +\frac{1}{15} A_{t}\left(\begin{array}{ccc}
45 & 25 \sqrt{2} & 0 \\
25 \sqrt{2} & -2 & 9 \sqrt{6} \\
0 & 9 \sqrt{6} & -43
\end{array}\right)+\frac{1}{15} A_{l d}\left(\begin{array}{ccc}
10 & 25 \sqrt{2} & 0 \\
25 \sqrt{2} & 12 & 9 \sqrt{6} \\
0 & 9 \sqrt{6} & -22
\end{array}\right) \\
& +b\left(\begin{array}{ccc}
-128 / 1125 & \sqrt{2} / 4 & -3 \sqrt{3} / 5 \\
\sqrt{2} / 4 & -146 / 1125 & 81 \sqrt{6} / 100 \\
-3 \sqrt{3} / 5 & 81 \sqrt{6} / 100 & 274 / 1125
\end{array}\right)
\end{aligned}
$$

There is a standard procedure to find the eigenvalues, $\lambda_{i}, i=1,2,3$, of the traceless symmetric $(3 \times 3)$ matrix. Denoting such a matrix as

$$
A=\left(\begin{array}{lll}
A_{11} & A_{12} & A_{13} \\
A_{12} & A_{22} & A_{23} \\
A_{13} & A_{23} & A_{33}
\end{array}\right),
$$

its characteristic equation

$$
\left(\lambda-\lambda_{1}\right)\left(\lambda-\lambda_{2}\right)\left(\lambda-\lambda_{3}\right)=\lambda^{3}-I_{1} \lambda^{2}-3 I_{2} \lambda-2 I_{3}=0,
$$


is expressed in terms of three rotational invariants:

$$
\begin{aligned}
& I_{1}=\operatorname{Tr}(A)=A_{11}+A_{22}+A_{33}=\lambda_{1}+\lambda_{2}+\lambda_{3}=0, \\
& I_{2}=\frac{1}{3}\left[A_{12}^{2}+A_{13}^{2}+A_{23}^{2}-A_{11} A_{22}-A_{11} A_{33}-A_{22} A_{33}\right]=-\frac{1}{3}\left(\lambda_{1} \lambda_{2}+\lambda_{1} \lambda_{3}+\lambda_{2} \lambda_{3}\right), \\
& I_{3}=\frac{1}{2} \operatorname{det}(A)=\frac{1}{2}\left(A_{11} A_{22} A_{33}+2 A_{12} A_{13} A_{23}-A_{11} A_{23}^{2}-A_{22} A_{13}^{2}-A_{33} A_{12}^{2}\right)=\frac{1}{2} \lambda_{1} \lambda_{2} \lambda_{3} .
\end{aligned}
$$

Since real eigenvalues are of our interest, two non-trivial invariants, $I_{2}$ and $I_{3}$, must satisfy the following conditions:

$$
I_{2}>0, \quad I_{2}^{3}>I_{3}^{2}
$$

If these conditions are fulfilled, we can define the angle:

$$
\phi=\frac{1}{3} \arccos \sqrt{\frac{I_{3}^{2}}{I_{2}^{3}}},
$$

through which the sorted eigenvalues $\left(\lambda_{1}>\lambda_{2}>\lambda_{3}\right)$ can be written as follows:

$$
\lambda_{1}=2 \sqrt{I_{2}} \cos (\phi), \quad \lambda_{2}=-2 \sqrt{I_{2}} \cos (\pi / 3+\phi), \quad \lambda_{3}=-2 \sqrt{I_{2}} \cos (\pi / 3-\phi) .
$$

After these eigenvalues are obtained, the masses are calculated easily:

$$
m_{11,12,13}^{P 1}=M_{0}+\frac{1}{4}\left(\mathcal{K}_{\bar{c} q}+\mathcal{K}_{\bar{c} c}\right)\left(2+r_{h d}+r_{l d}\right)+B-\frac{4}{3}\left(A_{t}+A_{l d}\right)-\frac{3622}{1125} b+\lambda_{3,2,1} .
$$

\section{B.2 The states with $J^{P}=3 / 2^{+}$and triquark spin $S_{t}=1 / 2$}

To derive the expressions for the masses of the four states with the spin-parity $J^{P}=3 / 2^{+}$ and triquark spin $S_{t}=1 / 2$ from table 5 , one needs to find the eigenvalues of the following non-diagonal symmetric $(4 \times 4)$ matrix:

$$
\begin{aligned}
& M_{J=3 / 2}^{P 1}=M_{0}+\frac{1}{2}\left(\mathcal{K}_{c q}\right)_{\overline{3}}\left(\begin{array}{cccc}
-3 & 0 & 0 & 0 \\
0 & -3 & 0 & 0 \\
0 & 0 & 1 & 0 \\
0 & 0 & 0 & 1
\end{array}\right)+\frac{1}{2}\left(\mathcal{K}_{q^{\prime} q^{\prime \prime}}\right)_{\overline{3}} \\
& +\frac{1}{2}\left(\mathcal{K}_{\bar{c} q}+\mathcal{K}_{\bar{c} c}\right)\left(\begin{array}{cccc}
0 & 0 & \sqrt{3} & 0 \\
0 & 0 & 0 & \sqrt{3} \\
\sqrt{3} & 0 & -2 & 0 \\
0 & \sqrt{3} & 0 & -2
\end{array}\right)+B-\frac{1}{3} A_{t}\left(\begin{array}{cccc}
1 & -2 \sqrt{5} & 0 & 0 \\
-2 \sqrt{5} & 2 & 0 & 0 \\
0 & 0 & 1 & -2 \sqrt{5} \\
0 & 0 & -2 \sqrt{5} & 2
\end{array}\right) \\
& +\frac{2}{3} A_{l d}\left(\begin{array}{cccc}
2 & \sqrt{5} & 0 & 0 \\
\sqrt{5} & -2 & 0 & 0 \\
0 & 0 & 2 & \sqrt{5} \\
0 & 0 & \sqrt{5} & -2
\end{array}\right)+\frac{1}{30} b\left(\begin{array}{cccc}
-40 & 3 \sqrt{5} & 0 & 0 \\
3 \sqrt{5} & -16 & 0 & 0 \\
0 & 0 & -40 & 3 \sqrt{5} \\
0 & 0 & 3 \sqrt{5} & -16
\end{array}\right) \text {. }
\end{aligned}
$$


Noting that the last three matrices are block-diagonal and their non-trivial $(2 \times 2)$ blocks are identical. A diagonalization of these matrices is reduced to the diagonalization of the blocks:

$$
\begin{gathered}
-\frac{A_{t}}{3}\left(\begin{array}{cc}
1 & -2 \sqrt{5} \\
-2 \sqrt{5} & 2
\end{array}\right)+\frac{2}{3} A_{l d}\left(\begin{array}{cc}
2 & \sqrt{5} \\
\sqrt{5} & -2
\end{array}\right)+\frac{b}{30}\left(\begin{array}{cc}
-40 & 3 \sqrt{5} \\
3 \sqrt{5} & -16
\end{array}\right) \\
\Longrightarrow-\frac{A_{t}}{2}-\frac{14}{15} b+\left(\begin{array}{cc}
\mu^{(3 / 2)} & 0 \\
0 & -\mu^{(3 / 2)}
\end{array}\right),
\end{gathered}
$$

where the following parameter is introduced:

$$
\mu^{(3 / 2)}=\frac{1}{30} \sqrt{\left(5 A_{t}+40 A_{l d}-12 b\right)^{2}+5\left(20 A_{t}+20 A_{l d}+3 b\right)^{2}} .
$$

Coming back to the $(4 \times 4)$ mass matrix $(B .12)$, it is necessary to point out that the transformation to the diagonal form of the sum of the last three matrices in (B.12) can be done by using the orthogonal matrix:

$$
a(\alpha)=\left(\begin{array}{cccc}
\cos \alpha & \sin \alpha & 0 & 0 \\
-\sin \alpha & \cos \alpha & 0 & 0 \\
0 & 0 & \cos \alpha & \sin \alpha \\
0 & 0 & -\sin \alpha & \cos \alpha
\end{array}\right)
$$

with some fixed value of the angle $\alpha$. Under such a transformation, the two matrices in the first row in (B.12) remain unchanged. Hence, the $(4 \times 4)$ mass matrix $($ B.12) can be presented in the form:

$$
\begin{aligned}
M_{J=3 / 2}^{P 1}= & M_{0}-\frac{1}{4}\left(\mathcal{K}_{\bar{c} q}+\mathcal{K}_{\bar{c} c}\right)\left(2+r_{h d}-r_{l d}\right)+B-\frac{A_{t}}{2}-\frac{14}{15} b+\frac{1}{2}\left(\mathcal{K}_{\bar{c} q}+\mathcal{K}_{\bar{c} c}\right) \\
& \times\left(\begin{array}{cccc}
1-r_{h d}+\tilde{\mu}^{(3 / 2)} & 0 & \sqrt{3} & 0 \\
0 & 1-r_{h d}-\tilde{\mu}^{(3 / 2)} & 0 & \sqrt{3} \\
\sqrt{3} & 0 & r_{h d}-1+\tilde{\mu}^{(3 / 2)} & 0 \\
0 & \sqrt{3} & 0 & r_{h d}-1-\tilde{\mu}^{(3 / 2)}
\end{array}\right),
\end{aligned}
$$

where $\tilde{\mu}^{(3 / 2)}=2 \mu^{(3 / 2)} /\left(\mathcal{K}_{\bar{c} q}+\mathcal{K}_{\bar{c} c}\right)$. Applying again the orthogonal transformations, the last matrix can be diagonalized, and we get the following set of masses:

$$
\begin{aligned}
& m_{7}^{P 1}=M_{0}+B-\frac{A_{t}}{2}-\frac{14}{15} b-\frac{1}{4}\left(\mathcal{K}_{\bar{c} q}+\mathcal{K}_{\bar{c} c}\right)\left(2+r_{h d}-r_{l d}+2 \sqrt{3+\left(1-r_{h d}\right)^{2}}\right)-\mu^{(3 / 2),} \\
& m_{8}^{P 1}=M_{0}+B-\frac{A_{t}}{2}-\frac{14}{15} b-\frac{1}{4}\left(\mathcal{K}_{\bar{c} q}+\mathcal{K}_{\bar{c} c}\right)\left(2+r_{h d}-r_{l d}+2 \sqrt{3+\left(1-r_{h d}\right)^{2}}\right)+\mu^{(3 / 2),}, \\
& m_{9}^{P 1}=M_{0}+B-\frac{A_{t}}{2}-\frac{14}{15} b-\frac{1}{4}\left(\mathcal{K}_{\bar{c} q}+\mathcal{K}_{\bar{c} c}\right)\left(2+r_{h d}-r_{l d}-2 \sqrt{3+\left(1-r_{h d}\right)^{2}}\right)-\mu^{(3 / 2),}, \\
& m_{10}^{P 1}=M_{0}+B-\frac{A_{t}}{2}-\frac{14}{15} b-\frac{1}{4}\left(\mathcal{K}_{\bar{c} q}+\mathcal{K}_{\bar{c} c}\right)\left(2+r_{h d}-r_{l d}-2 \sqrt{3+\left(1-r_{h d}\right)^{2}}\right)+\mu^{(3 / 2)} .
\end{aligned}
$$




\section{B.3 The states with $J^{P}=1 / 2^{+}$and triquark spin $S_{t}=1 / 2$}

For the first four states with the spin-parity $J^{P}=1 / 2^{+}$and triquark spin $S_{t}=1 / 2$ from table 5 , the mass matrix is again the non-diagonal symmetric $(4 \times 4)$ matrix:

$$
\begin{aligned}
& M_{J=1 / 2}^{P 1}=M_{0}+\frac{1}{2}\left(\mathcal{K}_{c q}\right)_{\overline{3}}\left(\begin{array}{cccc}
-3 & 0 & 0 & 0 \\
0 & -3 & 0 & 0 \\
0 & 0 & 1 & 0 \\
0 & 0 & 0 & 1
\end{array}\right)+\frac{1}{2}\left(\mathcal{K}_{q^{\prime} q^{\prime \prime}}\right)_{\overline{3}} \\
& +\frac{1}{2}\left(\mathcal{K}_{\bar{c} q}+\mathcal{K}_{\bar{c} c}\right)\left(\begin{array}{cccc}
0 & 0 & \sqrt{3} & 0 \\
0 & 0 & 0 & \sqrt{3} \\
\sqrt{3} & 0 & -2 & 0 \\
0 & \sqrt{3} & 0 & -2
\end{array}\right)+B+\frac{1}{3} A_{t}\left(\begin{array}{cccc}
2 & 2 \sqrt{2} & 0 & 0 \\
2 \sqrt{2} & -5 & 0 & 0 \\
0 & 0 & 2 & 2 \sqrt{2} \\
0 & 0 & 2 \sqrt{2} & -5
\end{array}\right) \\
& +\frac{2}{3} A_{l d}\left(\begin{array}{cccc}
-4 & \sqrt{2} & 0 & 0 \\
\sqrt{2} & -5 & 0 & 0 \\
0 & 0 & -4 & \sqrt{2} \\
0 & 0 & \sqrt{2} & -5
\end{array}\right)+\frac{1}{30} b\left(\begin{array}{cccc}
-16 & 21 \sqrt{2} & 0 & 0 \\
21 \sqrt{2} & -46 & 0 & 0 \\
0 & 0 & -16 & 21 \sqrt{2} \\
0 & 0 & 21 \sqrt{2} & -46
\end{array}\right) \text {. }
\end{aligned}
$$

The rotational matrix (B.15) with some fixed value of the angle $\alpha$ transforms the sum of the last three matrices to the diagonal form. As in the case of $J^{P}=3 / 2^{+}$pentaquarks, the diagonalization procedure can be applied to the $(2 \times 2)$ blocks:

$$
\begin{gathered}
\frac{A_{t}}{3}\left(\begin{array}{cc}
2 & 2 \sqrt{2} \\
2 \sqrt{2} & -5
\end{array}\right)+\frac{2}{3} A_{l d}\left(\begin{array}{cc}
-4 & \sqrt{2} \\
\sqrt{2} & -5
\end{array}\right)+\frac{b}{30}\left(\begin{array}{cc}
-16 & 21 \sqrt{2} \\
21 \sqrt{2} & -46
\end{array}\right) \\
\Longrightarrow-\frac{A_{t}}{2}-3 A_{l d}-\frac{31}{30} b+\left(\begin{array}{cc}
\mu^{(1 / 2)} & 0 \\
0 & -\mu^{(1 / 2)}
\end{array}\right)
\end{gathered}
$$

where the following parameter is introduced:

$$
\mu^{(1 / 2)}=\frac{1}{30} \sqrt{25\left(7 A_{t}+2 A_{l d}+3 b\right)^{2}+2\left(20 A_{t}+20 A_{l d}+21 b\right)^{2}} .
$$

After this transformation is done, the $(4 \times 4)$ mass matrix $($ B.21) takes the form:

$$
\begin{aligned}
M_{J=1 / 2}^{P 1}= & M_{0}-\frac{1}{4}\left(\mathcal{K}_{\bar{c} q}+\mathcal{K}_{\bar{c} c}\right)\left(2+r_{h d}-r_{l d}\right)+B-\frac{A_{t}}{2}-3 A_{l d}-\frac{31}{30} b+\frac{1}{2}\left(\mathcal{K}_{\bar{c} q}+\mathcal{K}_{\bar{c} c}\right) \\
& \times\left(\begin{array}{cccc}
1-r_{h d}+\tilde{\mu}^{(1 / 2)} & 0 & \sqrt{3} & 0 \\
0 & 1-r_{h d}-\tilde{\mu}^{(1 / 2)} & 0 & \sqrt{3} \\
\sqrt{3} & 0 & r_{h d}-1+\tilde{\mu}^{(1 / 2)} & 0 \\
0 & \sqrt{3} & 0 & r_{h d}-1-\tilde{\mu}^{(1 / 2)}
\end{array}\right),(\mathrm{B} .22)
\end{aligned}
$$

where $\tilde{\mu}^{(1 / 2)}=2 \mu^{(1 / 2)} /\left(\mathcal{K}_{\bar{c} q}+\mathcal{K}_{\bar{c} c}\right)$. The last matrix can be diagonalized by using the 


\begin{tabular}{|cccc|}
\hline Baryon & $J^{P}[59]$ & LHCb & Belle \\
\hline$\Omega_{c}(3000)$ & $1 / 2^{-}$ & $3000.4 \pm 0.2 \pm 0.1$ & $3000.7 \pm 1.0 \pm 0.2$ \\
$\Omega_{c}(3050)$ & $1 / 2^{-}$ & $3050.2 \pm 0.1 \pm 0.1$ & $3050.2 \pm 0.4 \pm 0.2$ \\
$\Omega_{c}(3066)$ & $3 / 2^{-}$ & $3065.5 \pm 0.1 \pm 0.3$ & $3064.9 \pm 0.6 \pm 0.2$ \\
$\Omega_{c}(3090)$ & $3 / 2^{-}$ & $3090.2 \pm 0.3 \pm 0.5$ & $3089.3 \pm 1.2 \pm 0.2$ \\
$\Omega_{c}(3119)$ & $5 / 2^{-}$ & $3119.0 \pm 0.3 \pm 0.9$ & $\ldots$ \\
\hline
\end{tabular}

Table 15. Experimental data from the LHCb [63] and Belle [64] collaborations. (Masses are given in $\mathrm{MeV}$.) All the measurements have the uncertainty ${ }_{-0.5}^{+0.3} \mathrm{MeV}$ coming from the mass of the groundstate $\Xi_{c}^{+}$-baryon, which is common in both analysis. The spin-parity, $J^{P}$, assignment assumes that all the states are orbitally-excited baryons as suggested in [59].

orthogonal transformations and we get the following set of masses:

$$
\begin{aligned}
m_{1}^{P 1}= & M_{0}+B-\frac{A_{t}}{2}-3 A_{l d}-\frac{31}{30} b \\
& -\frac{1}{4}\left(\mathcal{K}_{\bar{c} q}+\mathcal{K}_{\bar{c} c}\right)\left(2+r_{h d}-r_{l d}+2 \sqrt{3+\left(1-r_{h d}\right)^{2}}\right)-\mu^{(1 / 2)}, \\
m_{2}^{P 1}= & M_{0}+B-\frac{A_{t}}{2}-3 A_{l d}-\frac{31}{30} b \\
& -\frac{1}{4}\left(\mathcal{K}_{\bar{c} q}+\mathcal{K}_{\bar{c} c}\right)\left(2+r_{h d}-r_{l d}+2 \sqrt{3+\left(1-r_{h d}\right)^{2}}\right)+\mu^{(1 / 2)}, \\
m_{3}^{P 1}= & M_{0}+B-\frac{A_{t}}{2}-3 A_{l d}-\frac{31}{30} b \\
& -\frac{1}{4}\left(\mathcal{K}_{\bar{c} q}+\mathcal{K}_{\bar{c} c}\right)\left(2+r_{h d}-r_{l d}-2 \sqrt{3+\left(1-r_{h d}\right)^{2}}\right)-\mu^{(1 / 2)}, \\
m_{4}^{P 1}= & M_{0}+B-\frac{A_{t}}{2}-3 A_{l d}-\frac{31}{30} b \\
& -\frac{1}{4}\left(\mathcal{K}_{\bar{c} q}+\mathcal{K}_{\bar{c} c}\right)\left(2+r_{h d}-r_{l d}-2 \sqrt{3+\left(1-r_{h d}\right)^{2}}\right)+\mu^{(1 / 2)} .
\end{aligned}
$$

\section{C $\quad \chi^{2}$-analysis of orbitally-excited $\Omega_{c}^{*}$-baryons}

Existing experimental data on the masses of orbitally-excited $\Omega_{c}^{*}$-baryons were obtained by the LHCb [63] and Belle [64] collaborations and collected in table 15.

The theoretical expressions for the orbitally-exciting $\Omega_{c}^{*}$-baryon masses follow from the effective Hamiltonian [59]: ${ }^{7}$

$$
\begin{aligned}
& H_{\mathrm{eff}}=m_{c}+m_{\{s s\}}+2 \kappa_{s s}\left(\mathbf{S}_{s_{1}} \cdot \mathbf{S}_{s_{2}}\right)+\frac{B_{\mathcal{Q}}}{2} \mathbf{L}^{2}+V_{\mathrm{SD}}, \\
& V_{\mathrm{SD}}=2 a_{1}\left(\mathbf{L} \cdot \mathbf{S}_{\{s s\}}\right)+2 a_{2}\left(\mathbf{L} \cdot \mathbf{S}_{c}\right)+b \frac{S_{12}}{4}+2 c\left(\mathbf{S}_{\{s s\}} \cdot \mathbf{S}_{c}\right) .
\end{aligned}
$$

In eq. (C.1), $m_{c}$ and $m_{\{s s\}}$ are the masses of the $c$-quark and the spin-1 \{ss\}-diquark, respectively, $\kappa_{s s}$ is the spin-spin coupling of the quarks in the diquark, $\mathbf{L}$ is the orbital

\footnotetext{
${ }^{7}$ The coefficients of the spin-orbit, $a_{1}$ and $a_{2}$, and spin-spin, $c$, interactions in the spin-dependent part of the Hamiltonian, $V_{\mathrm{SD}}$, differ by a factor 2 from the ones defined in $[57,59]$.
} 
angular momentum of the $\Omega_{c}^{*}$-baryon, and $B_{\mathcal{Q}}$ is the orbital coupling. The coefficients $a_{1}$ and $a_{2}$ are the strengths of the spin-orbit terms involving the spin of the diquark $\mathbf{S}_{\{s s\}}$ and the charm-quark spin $\mathbf{S}_{c}$, respectively, $c$ is the strength of the spin-spin interaction between the diquark and the charm quark, and $S_{12} / 4$ represents the tensor interaction, defined by

$$
\frac{S_{12}}{4}=Q\left(\mathbf{S}_{\{s s\}}, \mathbf{S}_{c}\right)=3\left(\mathbf{S}_{\{s s\}} \cdot \mathbf{n}\right)\left(\mathbf{S}_{c} \cdot \mathbf{n}\right)-\left(\mathbf{S}_{\{s s\}} \cdot \mathbf{S}_{c}\right),
$$

where $\mathbf{n}=\mathbf{r} / r$ is the unit vector in the direction from charm quark to the doubly-strange diquark.

One should remember that several required internal parameters are fixed: charmquark spin $S_{c}=1 / 2$, diquark spin $S_{\{s s\}}=1$, and $\Omega_{c}^{*}$-baryon orbital angular momentum $L=1$. So, in the $L-S$ coupling scheme, two possible values of the total spin $S=1 / 2$ and $S=3 / 2$, after their coupling to $L$, allow to get five $P$-wave states: two with the total angular momentum $J=1 / 2$, two with $J=3 / 2$ and the last one with $J=5 / 2$ (see table 15 ). In this scheme the spin-dependent part, $V_{\mathrm{SD}}$, is represented by the block-diagonal matrix, each block of which corresponds to the states with a fixed value of $J$ [59]:

$$
\begin{aligned}
\Delta \mathcal{M}_{1 / 2} & =\frac{1}{3}\left(\begin{array}{rr}
2\left(a_{2}-4 a_{1}\right) & 2 \sqrt{2}\left(a_{2}-a_{1}\right) \\
2 \sqrt{2}\left(a_{2}-a_{1}\right) & -5\left(2 a_{1}+a_{2}\right)
\end{array}\right)+\frac{b}{\sqrt{2}}\left(\begin{array}{rr}
0 & 1 \\
1 & -\sqrt{2}
\end{array}\right)+c\left(\begin{array}{rr}
-2 & 0 \\
0 & 1
\end{array}\right) \\
\Delta \mathcal{M}_{3 / 2} & =\frac{1}{3}\left(\begin{array}{cc}
4 a_{1}-a_{2} & 2 \sqrt{5}\left(a_{2}-a_{1}\right) \\
2 \sqrt{5}\left(a_{2}-a_{1}\right) & -2\left(2 a_{1}+a_{2}\right)
\end{array}\right)+\frac{b}{10}\left(\begin{array}{rr}
0-\sqrt{5} \\
-\sqrt{5} & 8
\end{array}\right)+c\left(\begin{array}{rr}
-2 & 0 \\
0 & 1
\end{array}\right), \\
\Delta \mathcal{M}_{5 / 2} & =2 a_{1}+a_{2}-\frac{b}{5}+c .
\end{aligned}
$$

After diagonalizing the matrices and adding the common mass term

$$
M_{0}^{\left(\Omega_{c}\right)}=m_{c}+m_{\{s s\}}+\frac{\kappa_{s s}}{2}+B_{\mathcal{Q}},
$$

we get the set of five mass formulae:

$$
\begin{aligned}
m_{1}^{(1 / 2)}= & M_{0}^{\left(\Omega_{c}\right)}-\frac{1}{2}\left(6 a_{1}+a_{2}+b+c\right) \\
& -\frac{1}{6} \sqrt{\left(2 a_{1}+7 a_{2}+3 b-9 c\right)^{2}+2\left(4 a_{1}-4 a_{2}-3 b\right)^{2}}, \\
m_{2}^{(1 / 2)}= & M_{0}^{\left(\Omega_{c}\right)}-\frac{1}{2}\left(6 a_{1}+a_{2}+b+c\right) \\
& +\frac{1}{6} \sqrt{\left(2 a_{1}+7 a_{2}+3 b-9 c\right)^{2}+2\left(4 a_{1}-4 a_{2}-3 b\right)^{2}}, \\
m_{1}^{(3 / 2)}= & M_{0}^{\left(\Omega_{c}\right)}-\frac{1}{10}\left(5 a_{2}-4 b+5 c\right) \\
& -\frac{1}{30} \sqrt{\left(40 a_{1}+5 a_{2}-12 b-45 c\right)^{2}+5\left(20 a_{1}-20 a_{2}+3 b\right)^{2}}, \\
m_{2}^{(3 / 2)}= & M_{0}^{\left(\Omega_{c}\right)}-\frac{1}{10}\left(5 a_{2}-4 b+5 c\right) \\
& +\frac{1}{30} \sqrt{\left(40 a_{1}+5 a_{2}-12 b-45 c\right)^{2}+5\left(20 a_{1}-20 a_{2}+3 b\right)^{2},} \\
m^{(5 / 2)}= & M_{0}^{\left(\Omega_{c}\right)}+2 a_{1}+a_{2}-\frac{b}{5}+c .
\end{aligned}
$$




\begin{tabular}{|cccccc|}
\hline & $M_{0}^{\left(\Omega_{c}\right)}$ & $a_{1}$ & $a_{2}$ & $b$ & $c$ \\
\hline LHCb & $3079.89 \pm 0.40$ & $13.47 \pm 0.14$ & $12.86 \pm 0.38$ & $13.48 \pm 0.54$ & $2.00 \pm 0.22$ \\
Combined & $3079.80 \pm 0.39$ & $13.45 \pm 0.13$ & $12.94 \pm 0.36$ & $13.30 \pm 0.48$ & $2.01 \pm 0.20$ \\
\hline
\end{tabular}

Table 16. The $\chi^{2}$-analysis of the orbitally-excited $\Omega_{c}^{*}$-baryon masses based on the measurements by the $\mathrm{LHCb}$ and Belle collaborations. This analysis is performed based on the LHCb data alone and on the combined data set from both the $\mathrm{LHCb}$ and Belle collaborations presented in table 15 . All values of parameters are given in $\mathrm{MeV}$. The combine fit results $\chi_{\text {min }}^{2} / \mathrm{ndf}=0.87 / 4$.

There are five unknown variables $\left\{M_{0}^{\left(\Omega_{c}\right)}, a_{1}, a_{2}, b, c\right\}$ in these five equations. If there are five experimentally measured states which can be assigned with the $P$-wave $\Omega_{c}$-baryons, all five variables, in general, can be determined but their values do not always satisfy physical requirements.

After the assignment is done as specified in table 15 , it is possible to perform a $\chi^{2}$ analysis of the data. We start from the LHCb data alone as all five states are present there and then add the Belle measurements which allow to find $\chi_{\min }^{2}$ for four degrees of freedom. The best-fit values and $1 \sigma$ uncertainties of free parameters are presented in table 16 . There are no free degrees of freedom for the fit based on the $\mathrm{LHCb}$ data alone (the first row in table 16) but combining them with the Belle data results $\chi_{\min }^{2} / \mathrm{ndf}=0.87 / 4$.

The correlation matrices in both cases are as follows:

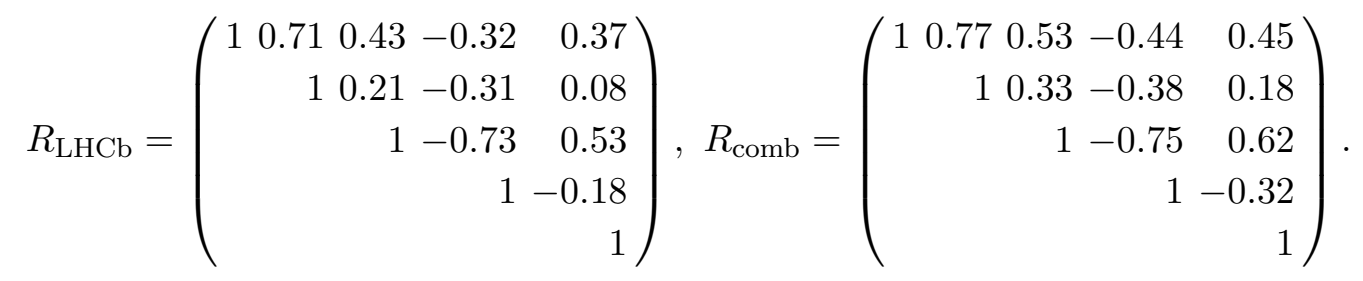

The common mass $M_{0}^{\left(\Omega_{c}\right)}$ is determined quite precisely. With the values of the input parameters from subsection 5.1: $m_{c}=(1710 \pm 10) \mathrm{MeV}, m_{\{s s\}}=(1099 \pm 15) \mathrm{MeV}$, and $\mathcal{K}_{s s}=(23 \pm 2) \mathrm{MeV}$, the strength of the orbital interaction can be estimated as:

$$
B_{\mathcal{Q}}=(259 \pm 18) \mathrm{MeV}
$$

Open Access. This article is distributed under the terms of the Creative Commons Attribution License (CC-BY 4.0), which permits any use, distribution and reproduction in any medium, provided the original author(s) and source are credited.

\section{References}

[1] LHCb collaboration, Observation of a narrow pentaquark state, $P_{c}(4312)^{+}$and of two-peak structure of the $P_{c}(4450)^{+}$, Phys. Rev. Lett. 122 (2019) 222001 [arXiv:1904.03947] [INSPIRE].

[2] LHCb collaboration, Observation of $J / \psi p$ Resonances Consistent with Pentaquark States in $\Lambda_{b}^{0} \rightarrow J / \psi K^{-} p$ Decays, Phys. Rev. Lett. 115 (2015) 072001 [arXiv: 1507.03414] [INSPIRE]. 
[3] Particle Data Group collaboration, Review of Particle Physics, Phys. Rev. D 98 (2018) 030001 [INSPIRE].

[4] A. Ali, J.S. Lange and S. Stone, Exotics: Heavy Pentaquarks and Tetraquarks, Prog. Part. Nucl. Phys. 97 (2017) 123 [arXiv: 1706.00610] [InSPIRE].

[5] A. Esposito, A. Pilloni and A.D. Polosa, Multiquark Resonances, Phys. Rept. 668 (2017) 1 [arXiv: 1611.07920] [INSPIRE].

[6] H.-X. Chen, W. Chen, X. Liu and S.-L. Zhu, The hidden-charm pentaquark and tetraquark states, Phys. Rept. 639 (2016) 1 [arXiv:1601.02092] [INSPIRE].

[7] F.-K. Guo, C. Hanhart, U.-G. Meissner, Q. Wang, Q. Zhao and B.-S. Zou, Hadronic molecules, Rev. Mod. Phys. 90 (2018) 015004 [arXiv: 1705.00141] [InSPIRE].

[8] S.L. Olsen, T. Skwarnicki and D. Zieminska, Nonstandard heavy mesons and baryons: Experimental evidence, Rev. Mod. Phys. 90 (2018) 015003 [arXiv: 1708.04012] [InSPIRE].

[9] R.F. Lebed, R.E. Mitchell and E.S. Swanson, Heavy-Quark QCD Exotica, Prog. Part. Nucl. Phys. 93 (2017) 143 [arXiv: 1610.04528] [InSPIRE].

[10] A. Hosaka, T. Iijima, K. Miyabayashi, Y. Sakai and S. Yasui, Exotic hadrons with heavy flavors: X, Y, Z and related states, PTEP 2016 (2016) 062C01 [arXiv:1603.09229] [INSPIRE].

[11] M. Karliner, J.L. Rosner and T. Skwarnicki, Multiquark States, Ann. Rev. Nucl. Part. Sci. 68 (2018) 17 [arXiv:1711.10626] [INSPIRE].

[12] C.-Z. Yuan, The XYZ states revisited, Int. J. Mod. Phys. A 33 (2018) 1830018 [arXiv: 1808.01570] [INSPIRE].

[13] Y.-R. Liu, H.-X. Chen, W. Chen, X. Liu and S.-L. Zhu, Pentaquark and Tetraquark states, Prog. Part. Nucl. Phys. 107 (2019) 237 [arXiv:1903.11976] [InSPIRE].

[14] N. Brambilla et al., The $X Y Z$ states: experimental and theoretical status and perspectives, arXiv: 1907.07583 [INSPIRE].

[15] A. Ali, L. Maiani and A.D. Polosa, Multiquark Hadrons, Cambridge University Press, Cambridge U.K. (2019).

[16] H.-X. Chen, W. Chen and S.-L. Zhu, Possible interpretations of the $P_{c}(4312), P_{c}(4440)$ and $P_{c}(4457)$, Phys. Rev. D 100 (2019) 051501 [arXiv:1903.11001] [INSPIRE].

[17] R. Chen, Z.-F. Sun, X. Liu and S.-L. Zhu, Strong LHCb evidence supporting the existence of the hidden-charm molecular pentaquarks, Phys. Rev. D 100 (2019) 011502 [arXiv: 1903.11013] [INSPIRE].

[18] F.-K. Guo, H.-J. Jing, U.-G. Meissner and S. Sakai, Isospin breaking decays as a diagnosis of the hadronic molecular structure of the $P_{c}(4457)$, Phys. Rev. D 99 (2019) 091501 [arXiv: 1903.11503] [INSPIRE].

[19] M.-Z. Liu et al., Emergence of a complete heavy-quark spin symmetry multiplet: seven molecular pentaquarks in light of the latest LHCb analysis, Phys. Rev. Lett. 122 (2019) 242001 [arXiv: 1903.11560] [INSPIRE].

[20] J. He, Study of $P_{c}(4457), P_{c}(4440)$ and $P_{c}(4312)$ in a quasipotential Bethe-Salpeter equation approach, Eur. Phys. J. C 79 (2019) 393 [arXiv:1903.11872] [INSPIRE]. 
[21] C.W. Xiao, J. Nieves and E. Oset, Heavy quark spin symmetric molecular states from $\bar{D}^{(*)} \Sigma_{c}^{(*)}$ and other coupled channels in the light of the recent LHCb pentaquarks, Phys. Rev. D 100 (2019) 014021 [arXiv:1904.01296] [INSPIRE].

$[22]$ H. Huang, J. He and J. Ping, Looking for the hidden-charm pentaquark resonances in $J / \psi p$ scattering, arXiv:1904.00221 [INSPIRE].

[23] Y. Shimizu, Y. Yamaguchi and M. Harada, Heavy quark spin multiplet structure of $P_{c}(4312)$, $P_{c}(4440)$ and $P_{c}(4457)$, arXiv: 1904.00587 [INSPIRE].

[24] C.-J. Xiao, Y. Huang, Y.-B. Dong, L.-S. Geng and D.-Y. Chen, Exploring the molecular scenario of Pc(4312), Pc(4440) and Pc(4457), Phys. Rev. D 100 (2019) 014022 [arXiv: 1904.00872] [INSPIRE].

[25] J.-R. Zhang, Exploring a $\Sigma_{c} \bar{D}$ state: with focus on $P_{c}(4312)^{+}$, arXiv:1904.10711 [INSPIRE].

[26] T. Skwarnicki, Recent results on exotic hadrons from LHCb, talk at the Workshop on Exotic Hadrons: Theory and Experiment at Lepton and Hadron Colliders, T.D. Lee Institute, Jiao-Tong University, Shanghai China (2019), https://indico.leeinst.sjtu.edu.cn/event/57/.

[27] JPAC collaboration, Interpretation of the LHCb $P_{c}(4312)^{+}$Signal, Phys. Rev. Lett. 123 (2019) 092001 [arXiv: 1904.10021] [INSPIRE].

[28] X.-Z. Weng, X.-L. Chen, W.-Z. Deng and S.-L. Zhu, Hidden-charm pentaquarks and $P_{c}$ states, Phys. Rev. D 100 (2019) 016014 [arXiv:1904.09891] [INSPIRE].

[29] GuveX collaboration, First Measurement of Near-Threshold $J / \psi$ Exclusive Photoproduction off the Proton, Phys. Rev. Lett. 123 (2019) 072001 [arXiv:1905.10811] [InSPIRE].

[30] A.N. Hiller Blin et al., Studying the $P_{c}(4450)$ resonance in $J / \psi$ photoproduction off protons, Phys. Rev. D 94 (2016) 034002 [arXiv: 1606.08912] [InSPIRE].

[31] X. Cao and J.-p. Dai, Confronting pentaquark photoproduction with new LHCb observations, Phys. Rev. D 100 (2019) 054033 [arXiv:1904.06015] [InSPIRE].

[32] X.-Y. Wang, X.-R. Chen and J. He, Possibility to study pentaquark states $P_{c}(4312), P_{c}(4440)$ and $P_{c}(4457)$ in $\gamma p \rightarrow J / \psi p$ reaction, Phys. Rev. D 99 (2019) 114007 [arXiv:1904.11706] [INSPIRE].

[33] M.B. Voloshin, Hidden-charm pentaquark formation in antiproton-deuterium collisions, Phys. Rev. D 99 (2019) 093003 [arXiv: 1903.04422] [INSPIRE].

[34] A. Ali and A. Ya. Parkhomenko, Interpretation of the narrow $J / \psi p$ Peaks in $\Lambda_{b} \rightarrow J / \psi p K^{-}$ decay in the compact diquark model, Phys. Lett. B 793 (2019) 365 [arXiv:1904.00446] [INSPIRE].

[35] L. Maiani, F. Piccinini, A.D. Polosa and V. Riquer, Diquark-antidiquarks with hidden or open charm and the nature of X(3872), Phys. Rev. D 71 (2005) 014028 [hep-ph/0412098] [INSPIRE].

[36] H.J. Lipkin, New Possibilities for Exotic Hadrons: Anticharmed Strange Baryons, Phys. Lett. B 195 (1987) 484 [INSPIRE].

[37] R.L. Jaffe and F. Wilczek, Diquarks and exotic spectroscopy, Phys. Rev. Lett. 91 (2003) 232003 [hep-ph/0307341] [INSPIRE].

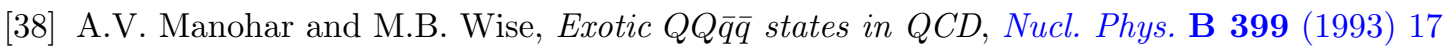
[hep-ph/9212236] [INSPIRE]. 
[39] A. Esposito, M. Papinutto, A. Pilloni, A.D. Polosa and N. Tantalo, Doubly charmed tetraquarks in $B_{c}$ and $\Xi_{b c}$ decays, Phys. Rev. D 88 (2013) 054029 [arXiv:1307.2873] [INSPIRE].

[40] S.-Q. Luo, K. Chen, X. Liu, Y.-R. Liu and S.-L. Zhu, Exotic tetraquark states with the $q q \bar{Q} \bar{Q}$ configuration, Eur. Phys. J. C 77 (2017) 709 [arXiv:1707.01180] [INSPIRE].

[41] M. Karliner and J.L. Rosner, Discovery of doubly-charmed $\Xi_{c c}$ baryon implies a stable (bbū $\left.\bar{d}\right)$ tetraquark, Phys. Rev. Lett. 119 (2017) 202001 [arXiv:1707.07666] [INSPIRE].

[42] E.J. Eichten and C. Quigg, Heavy-quark symmetry implies stable heavy tetraquark mesons $Q_{i} Q_{j} \bar{q}_{k} \bar{q}_{l}$, Phys. Rev. Lett. 119 (2017) 202002 [arXiv:1707.09575] [INSPIRE].

[43] A. Francis, R.J. Hudspith, R. Lewis and K. Maltman, Lattice Prediction for Deeply Bound Doubly Heavy Tetraquarks, Phys. Rev. Lett. 118 (2017) 142001 [arXiv:1607. 05214] [INSPIRE].

[44] P. Bicudo, M. Cardoso, A. Peters, M. Pflaumer and M. Wagner, ud $\bar{b} \bar{b}$ tetraquark resonances with lattice QCD potentials and the Born-Oppenheimer approximation, Phys. Rev. D 96 (2017) 054510 [arXiv: 1704.02383] [inSPIRE].

[45] P. Junnarkar, M. Padmanath and N. Mathur, Heavy light tetraquarks from Lattice QCD, EPJ Web Conf. 175 (2018) 05014 [arXiv:1712.08400] [INSPIRE].

[46] T. Mehen, Implications of Heavy Quark-Diquark Symmetry for Excited Doubly Heavy Baryons and Tetraquarks, Phys. Rev. D 96 (2017) 094028 [arXiv:1708.05020] [INSPIRE].

[47] A. Czarnecki, B. Leng and M.B. Voloshin, Stability of tetrons, Phys. Lett. B 778 (2018) 233 [arXiv: 1708.04594] [INSPIRE].

[48] L. Maiani, A.D. Polosa and V. Riquer, Hydrogen bond of QCD, Phys. Rev. D 100 (2019) 014002 [arXiv: 1903.10253] [INSPIRE].

[49] L. Maiani, A.D. Polosa and V. Riquer, Hydrogen bond of QCD in doubly heavy baryons and tetraquarks, Phys. Rev. D 100 (2019) 074002 [arXiv:1908.03244] [InSPIRE].

[50] R.F. Lebed, The Pentaquark Candidates in the Dynamical Diquark Picture, Phys. Lett. B 749 (2015) 454 [arXiv: 1507.05867] [INSPIRE].

[51] A. Ali, I. Ahmed, M.J. Aslam and A. Rehman, Heavy quark symmetry and weak decays of the b-baryons in pentaquarks with a ç component, Phys. Rev. D 94 (2016) 054001 [arXiv: 1607.00987] [INSPIRE].

[52] A. Ali, I. Ahmed, M.J. Aslam and A. Rehman, Mass spectrum of spin-1/2 pentaquarks with a c $\bar{c}$ component and their anticipated discovery modes in b-baryon decays, arXiv:1704.05419 [INSPIRE].

[53] G.-N. Li, X.-G. He and M. He, Some Predictions of Diquark Model for Hidden Charm Pentaquark Discovered at the LHCb, JHEP 12 (2015) 128 [arXiv: 1507.08252] [INSPIRE].

[54] J.F. Giron, R.F. Lebed and C.T. Peterson, The Dynamical Diquark Model: First Numerical Results, JHEP 05 (2019) 061 [arXiv: 1903.04551] [INSPIRE].

[55] M.I. Eides, V.Y. Petrov and M.V. Polyakov, New LHCb pentaquarks as hadrocharmonium states, arXiv:1904.11616 [INSPIRE].

[56] L. Maiani, F. Piccinini, A.D. Polosa and V. Riquer, The Z(4430) and a New Paradigm for Spin Interactions in Tetraquarks, Phys. Rev. D 89 (2014) 114010 [arXiv:1405.1551] [INSPIRE]. 
[57] A. Ali et al., A new look at the $Y$ tetraquarks and $\Omega_{c}$ baryons in the diquark model, Eur. Phys. J. C 78 (2018) 29 [arXiv:1708.04650] [InSPIRE].

[58] L.D. Landau and E.M. Lifshitz, Quantum Mechanics (Nonrelativistic Theory), third edition, Pergamon Press, Oxford U.K. (1977).

[59] M. Karliner and J.L. Rosner, Very narrow excited $\Omega_{c}$ baryons, Phys. Rev. D 95 (2017) 114012 [arXiv: 1703.07774] [INSPIRE].

[60] A.R. Edmonds, Angular Momentum in Quantum Mechanics, Princeton University Press, Princeton U.S.A. (1957).

[61] M. Karliner and J.L. Rosner, Baryons with two heavy quarks: Masses, production, decays and detection, Phys. Rev. D 90 (2014) 094007 [arXiv: 1408.5877] [INSPIRE].

[62] M. Karliner and J.L. Rosner, Scaling of P-wave excitation energies in heavy-quark systems, Phys. Rev. D 98 (2018) 074026 [arXiv: 1808.07869] [InSPIRE].

[63] LHCb collaboration, Observation of five new narrow $\Omega_{c}^{0}$ states decaying to $\Xi_{c}^{+} K^{-}$, Phys. Rev. Lett. 118 (2017) 182001 [arXiv:1703.04639] [INSPIRE].

[64] Belle collaboration, Observation of Excited $\Omega_{c}$ Charmed Baryons in $e^{+} e^{-}$Collisions, Phys. Rev. D 97 (2018) 051102 [arXiv: 1711.07927] [INSPIRE].

[65] F. Stancu, Spectrum of the uudcc hidden charm pentaquark with an SU(4) flavor-spin hyperfine interaction, arXiv: 1902.07101 [INSPIRE].

[66] L. Maiani, A.D. Polosa and V. Riquer, A Theory of X and $Z$ Multiquark Resonances, Phys. Lett. B 778 (2018) 247 [arXiv:1712.05296] [INSPIRE].

[67] S.J. Brodsky, D.S. Hwang and R.F. Lebed, Dynamical Picture for the Formation and Decay of the Exotic XYZ Mesons, Phys. Rev. Lett. 113 (2014) 112001 [arXiv:1406.7281] [INSPIRE].

[68] R.F. Lebed, Spectroscopy of Exotic Hadrons Formed from Dynamical Diquarks, Phys. Rev. D 96 (2017) 116003 [arXiv: 1709.06097] [INSPIRE].

[69] A. Esposito and A.D. Polosa, A bbb̄b di-bottomonium at the LHC?, Eur. Phys. J. C 78 (2018) 782 [arXiv:1807.06040] [INSPIRE].

[70] H. An and M.B. Wise, The Direct Coupling of Light Quarks to Heavy Di-quarks, Phys. Lett. B 788 (2019) 131 [arXiv: 1809.02139] [INSPIRE]. 